\title{
High-Speed Decoders for Polar Codes
}

\author{
Pascal Giard
}

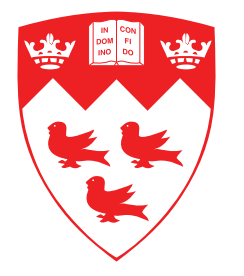

Department of Electrical and Computer Engineering

McGill University

Montreal, Canada

September 2016

A thesis submitted to McGill University in partial fulfillment of the requirements for the degree of Doctor of Philosophy.

(C) 2016 Pascal Giard 



\section{Acknowledgments}

I would like to start by thanking my supervisors, Warren J. Gross and Claude Thibeault. Thanks for their continuous support, mentorship, valuable advice and helpful discussions provided over the years. I am glad that we had such a good relationship that allowed me to freely explore while staying focused on tangible goals.

Many thanks to my friend and colleague Gabi Sarkis. A lot of this work would have been tremendously more difficult to nearly impossible without his help. His algorithmic, software and hardware skills, his vast knowledge, and his insightful comments were all of incredible help. Furthermore, his willingness to cooperate led to very fruitful collaborations stirring both of us up and helping me to remain motivated during the harder times.

I would also like to thank Alexandre J. Raymond, Alexios Balatsoukas-Stimming and Carlo Condo who helped me in one way or another. Thanks to Samuel Gagné, Marwan Kanaan and François Leduc-Primeau for the interesting discussions we had during our downtime.

I am grateful for the financial support I got from the Fonds Québécois de la Recherche sur la Nature et les Technologies, the fondation Pierre Arbour and the Regroupement Stratégique en Microsystèmes du Québec.

Finally, I would like to thank my beautiful boys Freddo and Gouri as well as my wonderful and beloved Joëlle. Their patience, support and indefectible love made this possible. Countless times, Joëlle had to sacrifice or take everything on her shoulders so that I could pursue this degree, and the one before. I am very grateful and privileged that she stayed by my side. 


\begin{abstract}
Error detection and correction plays a vital role in modern information storage and communication systems. Polar codes are gathering a lot of attention as they are a class of capacity-achieving errorcorrecting codes with an explicit construction that can be decoded with low-complexity algorithms. However, their adoption is hindered by the lack of high-speed-high throughput and low latencyhardware and software decoders for codes of practical length and rate.

This thesis presents various solutions to this problem. It introduces modifications to the stateof-the-art low-complexity decoding algorithm to better accommodate low-rate polar codes. It also proposes a code construction alteration process. Hardware implementation results show good latency reduction and throughput improvement with little to negligible coding loss for low-rate moderate-length polar codes.

Then, it presents high-speed software polar decoders. It shows how adapting the decoding algorithm at various levels can lead to significant improvements in latency and throughput, yielding polar decoders that are suitable for high-performance software-defined radio applications on modern desktop processors and embedded-platform processors. These proposed decoders have an order of magnitude lower latency and memory footprint compared to state-of-the-art decoders, while maintaining comparable throughput. In addition, strategies and results for implementing polar decoders on graphical processing units are presented.

Next, it demonstrates that polar decoders can achieve extremely high throughput values and retain moderate complexity. It presents a family of architectures for hardware polar decoders that employ unrolling. The resulting fully-unrolled architectures are capable of achieving a throughput that is two to three orders of magnitude greater than current state of the art while maintaining good energy efficiency. Moreover, the proposed architectures are flexible in a way that makes it possible to explore the trade-off between area, throughput and energy efficiency.

Lastly, while unrolled decoders provide the greatest decoding speed, they are built for a specific, fixed, code i.e. the code length or rate cannot be modified at execution time. Most modern wireless communication applications largely benefit from the support of multiple code lengths and rates. This thesis shows how an unrolled decoder can be transformed into a multi-mode decoder supporting many codes of various lengths and rates. Implementation results show a peak information throughput that is an order of magnitude greater than the state of the art, while showing the best area and energy efficiency.
\end{abstract}




\begin{abstract}
Abrégé
La détection et la correction des erreurs jouent un rôle essentiel dans les systèmes modernes de stockage et de communication. Les codes polaires intriguent actuellement beaucoup de chercheurs car ils constituent une classe de codes correcteurs capables d'atteindre la capacité théorique d'un canal avec des algorithmes de décodage de faible complexité tout en proposant une méthode de construction explicite. Cependant, leur adoption est ralentie par le manque d'implémentation matérielle et logicielle de décodeurs hautes vitesses i.e. à faible latence et à haut débit.

Cette thèse propose de multiples solutions à ce problème. Elle introduit d'abord des modifications à l'algorithme de décodage de faible complexité, qui est l'état de l'art, afin d'accommoder les codes polaires à faible taux de codage. Elle propose également une méthode d'altération de la construction des codes polaires. Les résultats d'implémentation matérielle montrent que, pour des codes polaires de longueur moyenne et de faible taux de codage, on obtient une bonne réduction de la latence ainsi qu'une augmentation appréciable du débit au coût d'une perte faible ou nulle en terme de performance de correction d'erreurs.

Puis, elle présente des décodeurs polaires logiciels hautes vitesses. Elle montre, qu'en adaptant l'algorithme de décodage à divers niveaux, on obtient des améliorations significatives en terme de latence et de débit. Il en résulte des décodeurs polaires très intéressants pour les applications de radio logicielle haute performance s'exécutant sur processeur moderne de bureau ou de plate-forme embarquée. Les décodeurs proposés ont une latence et une empreinte mémoire qui est un ordre de grandeur inférieur par rapport à l'état de l'art tout en maintenant un débit compétitif. De plus, des stratégies ainsi que des résultats pour l'implémentation de décodeurs polaires sont présentés pour des processeurs graphiques généralistes.

Ensuite, elle démontre que les décodeurs de codes polaires peuvent atteindre des débits excessivement élevés tout en conservant une complexité modérée. Elle présente une famille d'architecture matérielle pour les décodeurs de code polaire faisant appel à la technique de déroulage. Les architectures complètement déroulées qui en résultent sont capables d'atteindre des débits qui sont de deux à trois fois plus élevés que l'état de l'art tout en maintenant une bonne efficacité énergétique. De plus, les architectures proposées sont flexibles de sorte qu'il est possible d'explorer les compromis entre la surface, le débit et l'efficacité énergétique.

Enfin, bien que les décodeurs déroulés offrent la meilleure vitesse, ils sont construits pour un code spécifique i.e. un code d'une longueur et d'un taux de codage qui ne peuvent être modifiés au
\end{abstract}


moment de l'exécution. Les systèmes de communication sans-fil modernes bénéficient du support de multiple codes de longueurs et de taux variés. Ainsi, cette thèse montre comment un décodeur déroulé peut être transformé en décodeur multimode supportant plusieurs codes de longueurs et de taux variés. Les résultats d'implémentation montrent un débit nominal qui est un ordre de grandeur plus élevé que l'état de l'art tout en montrant les meilleurs taux d'efficacité en terme de surface et d'énergie. 


\section{Contents}

Contents

List of Figures $\quad$ xi

List of Tables $\quad$ xiii

1 Introduction $\quad 1$

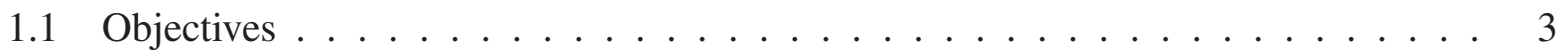

1.2 Summary of Thesis Contributions _. . . . . . . . . . . . . 3

1.3 Related Publications . . . . . . . . . . . . . . . . . 5

1.4 Thesis Organization . . . . . . . . . . . . . . . . . . 7

2 Polar Codes $\quad 9$

2.1 Construction . . . . . . . . . . . . . . . . . . . . 9

2.2 Tree Representation . . . . . . . . . . . . . . . . . . . . . 10

2.3 Systematic Coding . . . . . . . . . . . . . . . . . . 11

2.4 Successive-Cancellation Decoding . . . . . . . . . . . . . . . . 12

2.5 Simplified Successive-Cancellation Decoding . . . . . . . . . . . . . . 13

2.5.1 Rate-0 Nodes . . . . . . . . . . . . . . . . . . . 13

2.5.2 Rate-1 Nodes . . . . . . . . . . . . . . . . . . 13

2.5.3 Rate-R Nodes . . . . . . . . . . . . . . . . . . . 14

2.6 Fast-SSC Decoding . . . . . . . . . . . . . . . . . . . . 14

2.6.1 Repetition codes ........................... 14

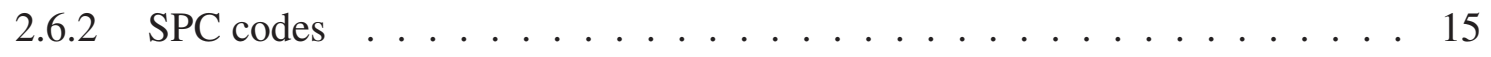

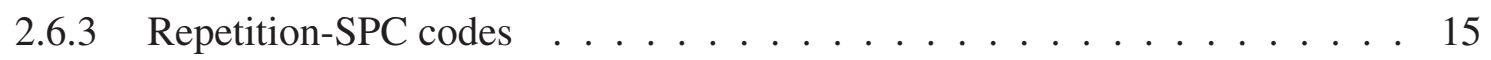


2.6 .4 Other Operations . . . . . . . . . . . . . . . 16

2.7 Other SC-based Decoding Algorithms . . . . . . . . . . . . . 16

2.7.1 ML-SSC Decoding . . . . . . . . . . . . . . . 16

2.7.2 Hybrid ML-SC Decoding . . . . . . . . . . . . . . 16

2.8 Other Decoding Algorithms _. . . . . . . . . . . . . . . . . 17

2.8.1 Belief-Propagation Decoding . . . . . . . . . . . . 17

2.8.2 List-based Decoding . . . . . . . . . . . . . . . . . . 17

2.9 SC-based Decoder Hardware Implementations . . . . . . . . . . . . . . . . . 19

2.9.1 Processing Element for SC Decoding . . . . . . . . . . . . . . . . 19

2.9.2 Semi-Parallel Decoder . . . . . . . . . . . . . . . 20

2.9 .3 Two-Phase Decoder . . . . . . . . . . . . . . . 20

2.9.4 Processor-like Decoder or the Original Fast-SSC Decoder . . . . . . . . . 20

2.9 .5 Implementation Results . . . . . . . . . . . . . . . . 20

3 Fast Low-Complexity Hardware Decoders for Low-Rate Polar Codes 23

3.1 Introduction . . . . . . . . . . . . . . . . . . 23

3.2 Altering the Code Construction . . . . . . . . . . . . . . . . 24

3.2.1 Original Construction . . . . . . . . . . . . . . . 24

3.2.2 Altered Polar Code Construction . . . . . . . . . . . . . . . 25

3.2.3 Proposed Altered Construction . . . . . . . . . . . . . . . . . 26

3.3 New Constituent Decoders . . . . . . . . . . . . . . . . . . . 30

3.4 Implementation . . . . . . . . . . . . . . . . 33

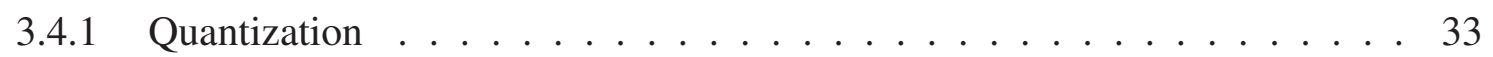

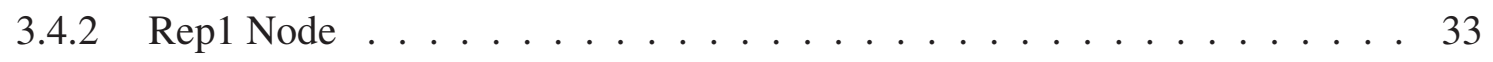

3.4.3 High-Level Architecture . . . . . . . . . . . . . . . . 34

3.4.4 Processing Unit or Processor . . . . . . . . . . . . . . . . . . 35

3.5 Results . . . . . . . . . . . . . . . . . . . 35

3.5 .1 Verification Methodology . . . . . . . . . . . 35

3.5.2 Comparison with State-of-the-art Decoders . . . . . . . . . . . . 36

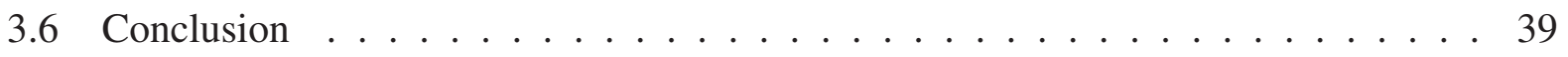

4 Low-Latency Software Polar Decoders 41

4.1 Introduction . . . . . . . . . . . . . . . . . . . . . . 41 
4.2 Implementation on $\mathrm{x} 86$ Processors $\ldots \ldots \ldots . \ldots . \ldots . \ldots . \ldots 43$

4.2.1 Instruction-based Decoder . . . . . . . . . . . . . . . . . . . . 44

4.2.2 Unrolled Decoder . . . . . . . . . . . . . . . . . . . . . . . . . . . . . 47

4.3 Implementation on Embedded Processors _ . . . . . . . . . . . . . . . . 54

4.4 Implementation on Graphical Processing Units . . . . . . . . . . . . . 55

4.4.1 Overview of the GPU Architecture and Terminology . . . . . . . . . . 55

4.4.2 Choosing an Appropriate Number of Threads per Block . . . . . . . . 56

4.4.3 Choosing an Appropriate Number of Blocks per Kernel . . . . . . . . . . 56

4.4.4 On the Constituent Codes Implemented . . . . . . . . . . . . . . 57

4.4.5 Shared Memory and Memory Coalescing _ . . . . . . . . . . . 58

4.4.6 Asynchronous Memory Transfers and Multiple Streams _. . . . . . . . 58

4.4.7 On the Use of Fixed-Point Numbers on a GPU . . . . . . . . . . . . 59

4.4 .8 Results . . . . . . . . . . . . . . . . . . . . . . 59

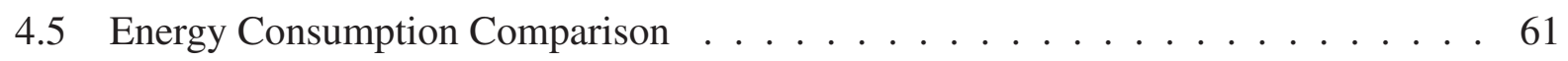

4.6 Further Discussion $\ldots \ldots \ldots \ldots$. . . . . . . . . . . . . . 62

4.6.1 On the relevance of the instruction-based decoders . . . . . . . . . 62

4.6.2 On the relevance of software decoders in comparison to hardware decoders 62

4.6 .3 Comparison with LDPC codes . . . . . . . . . . . . . . . 63

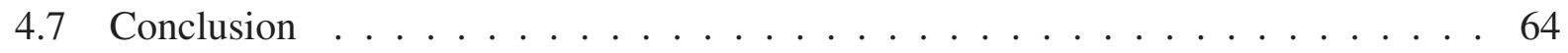

5 Unrolled Hardware Architectures for Polar Decoders $\quad 67$

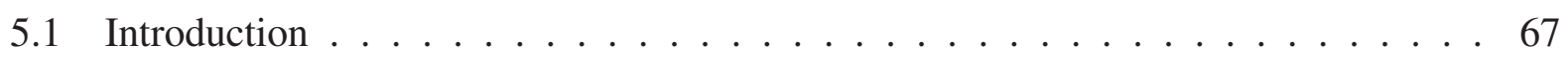

5.2 State-of-the-Art Architectures with Implementations $\ldots \ldots$. . . . . . . . 68

5.3 Architecture, Operations and Processing Nodes . . . . . . . . . . . . . . 69

5.3.1 Fully Unrolled (Basic Scheme) . . . . . . . . . . . . . . . . . . . . . . 69

5.3 .2 Deeply Pipelined . . . . . . . . . . . . . . . . . . . . 71

5.3 .3 Partially Pipelined . . . . . . . . . . . . . . . . 72

5.3 .4 Operations and Processing Nodes . . . . . . . . . . . . . . 74

5.3.5 Replacing Register Chains with SRAM Blocks . . . . . . . . . . . 75

5.4 Implementation and Results $\ldots \ldots \ldots \ldots \ldots$

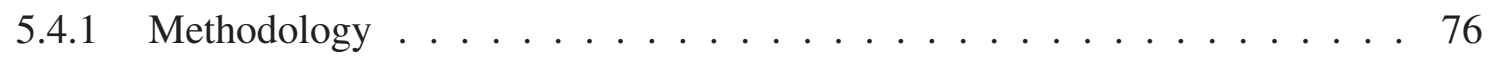

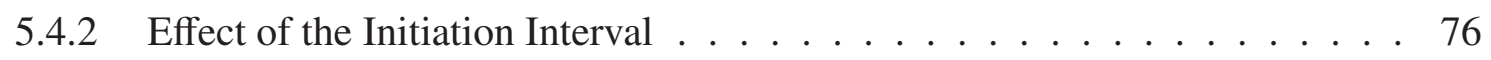


5.4.3 Comparison with State-of-the-Art Decoders . . . . . . . . . . . . 78

5.4.4 Effect of the Code Length and Rate . . . . . . . . . . . . . . . . . 80

5.4.5 On the Use of Code Shortening in an Unrolled Decoder . . . . . . . . . . . 84

5.4 .6 I/O Bounded Decoding . . . . . . . . . . . . . . 85

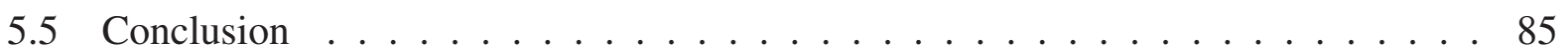

6 Multi-mode Unrolled Polar Decoding $\quad 87$

6.1 Introduction . . . . . . . . . . . . . . . . . . . . 87

6.2 Polar Code Example and its Decoder Tree Representations . . . . . . . . . . . . 88

6.3 Unrolled Architectures . . . . . . . . . . . . . . . . . . . . . . . . . . . 89

6.4 Multi-mode Unrolled Decoders . . . . . . . . . . . . . . . . . . . . . . 89

6.4.1 Hardware Modifications to the Unrolled Decoders . . . . . . . . . . . . 90

6.4.2 On the Construction of the Master Code . . . . . . . . . . . . . . . 90

6.4.3 About Constituent Codes: frozen bit locations, rate and practicality . . . . 92

6.4.4 Latency and Throughput Considerations . . . . . . . . . . . . . . . 93

6.5 Implementation Results . . . . . . . . . . . . . . . . . . . . . . 94

6.5.1 Error-correction Performance . . . . . . . . . . . . . 95

6.5.2 Latency and Throughput . . . . . . . . . . . . . . . 96

6.5.3 Synthesis Results and Comparison with the State of the Art . . . . . . . . 97

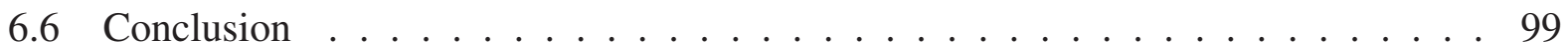

7 Conclusion and Future Work $\quad 101$

7.1 Future Work . . . . . . . . . . . . . . . . . . . . 102

7.1.1 Software Encoding and Decoding on APU Processors . . . . . . . . . . 103

7.1.2 Software Encoding and Decoding on Micro-controllers . . . . . . . . . . 103

7.1.3 High-speed Systematic Encoder . . . . . . . . . . . . . . . . . . 103

7.1.4 Multi-mode Unrolled List Decoders . . . . . . . . . . . . . . . . . . . . . 104

$\begin{array}{ll}\text { List of Acronyms } & 113\end{array}$ 


\section{List of Figures}

2.1 Construction of polar codes of lengths 2 and $4 \ldots \ldots$. . . . . . . . 10

2.2 Non-systematic $(8,4)$ polar code represented as a graph and as a decoder tree. . . . 11

2.3 Low-complexity systematic encoding of a $(8,4)$ polar code. . . . . . . . . . . . 12

2.4 Decoder trees corresponding to the SC, SSC and Fast-SSC decoding algorithms. . . 14

2.5 Error-correction performance of BP and SC decoding for a $(2048,1723)$ polar code 18

2.6 Error-correction performance of List, List-CRC and SC decoding of a (2048, 1723) polar code versus that of the $(1944,1620) 802.11$ n LDPC code. . . . . . . . . . . . 19

2.7 Architecture of the data processing unit proposed in [8] . . . . . . . . . . . 21

3.1 Decoder tree for the $(1024,512)$ polar code built using [22] and decoded with the nodes and operations of Table 3.1. . . . . . . . . . . . . . 26

3.2 Decoder trees for two different $(512,376)$ polar codes, where (a) and (b) are before and after construction alteration, respectively. . . . . . . . . . . . . . . . 29

3.3 Decoder tree for the altered $(1024,512)$ polar code . . . . . . . . . . . . . 30

3.4 Error-correction performance of the altered codes compared to that of the original codes constructed using the Tal and Vardy method. . . . . . . . . . . . . . . 31

3.5 Decoder tree for the altered polar code with the added nodes. . . . . . . . . . . 32

3.6 Impact of quantization on the error-correction performance of the proposed $(1024,512)$ polar code. . . . . . . . . . . . . . . . . . 33

3.7 Architecture of the Rep1 Node. . . . . . . . . . . . . . . . . . . . 34

3.8 High-level architecture of the decoder. . . . . . . . . . . . . . . . . . . 35

3.9 Architecture of the processing unit . . . . . . . . . . . . . . 36

4.1 Effect of quantization on error-correction performance. . . . . . . . . . . 45 
4.2 Dataflow graph of a $(8,5)$ polar decoder . . . . . . . . . . . . . . 48

4.3 Polar decoding on GPU: Effect of the number of threads per block. . . . . . . . . . 56

4.4 Polar decoding on GPU: Effect of the number of blocks per kernel. . . . . . . . . . 57

4.5 Polar decoding on GPU: Shared versus global memory. . . . . . . . . . . . . . . 58

4.6 Polar codes compared with LDPC codes from the 802.11n standard. . . . . . . . . 64

5.1 Decoder trees for an $(8,4)$ polar code decoded with the (a) SSC and (b) Fast-SSC

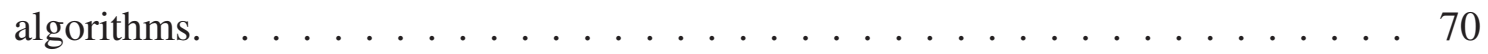

5.2 Fully-unrolled decoder for a $(8,4)$ polar code . . . . . . . . . . . . . . 70

5.3 Fully-unrolled deeply-pipelined decoder for a $(8,4)$ polar code . . . . . . . . . . . 71

5.4 Fully-unrolled deeply-pipelined decoder for a $(16,14)$ polar code. . . . . . . . . 72

5.5 Fully-unrolled partially-pipelined decoder for a $(16,14)$ polar code with $\mathcal{I}=2$. . . 73

5.6 Effect of quantization on the error-correction performance of a polar code. . . . . 77

5.7 Maximum FPGA resource usage and coded throughput of unrolled polar decoders. 84

6.1 Decoder trees for SC (a) and Fast-SSC (b) decoding of a $(16,12)$ polar code. . . . 88

6.2 Unrolled partially-pipelined decoder for a $(16,12)$ polar code with initiation interval $\mathcal{I}=2 \ldots \ldots \ldots \ldots$. . . . . . . . . . . . . . . . . . . . . 89

6.3 Error-correction performance of two $(2048,1365)$ polar codes with different constructions. . . . . . . . . . . . . . . . . . . . . 91

6.4 Error-correction performance of the four constituent codes of length 128 with a rate of approximately $5 / 6$ contained in the proposed $(2048,1365)$ master code. . . . 93

6.5 Error-correction performance of the polar codes. . . . . . . . . . . . . . . . 95 


\section{List of Tables}

2.1 Post-fitting results for SC-based decoder implementations. . . . . . . . . . . . 22

2.2 Latency and information throughput for SC-based decoder implementations. . . . . 22

3.1 Decoder tree node types supported by the original Fast-SSC polar decoder [8] . . . 25

3.2 New functions performed by the proposed decoder. . . . . . . . . . . . . . 31

3.3 Frozen bit patterns decoded by leaf nodes. . . . . . . . . . . . . . . . 32

3.4 Post-fitting results for rate-flexible decoders for moderate-length polar codes. . . . 37

3.5 Latency and information throughput comparison for low-rate moderate-length polar codes. . . . . . . . . . . . . . . . . . . . . 38

3.6 Comparison of state-of-the-art ASIC decoders decoding a $(1024,512)$ polar code. . 39

4.1 Decoding polar codes with the instruction-based decoder. . . . . . . . . . . . 47

4.2 Decoding polar codes with floating-point precision using SIMD, comparing the instruction-based decoder (ID) with the unrolled decoder (UD) . . . . . . . . . . . 52

4.3 Comparison of the proposed software decoder with that of [49]. . . . . . . . . . 53

4.4 Effect of unrolling and algorithm choice on decoding speed of the $(2048,1707)$

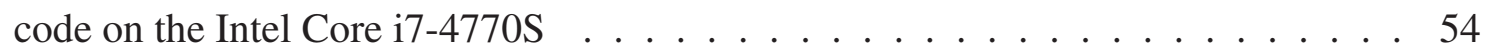

4.5 Decoding polar codes with 8-bit fixed-point numbers on an ARM Cortex A9 using

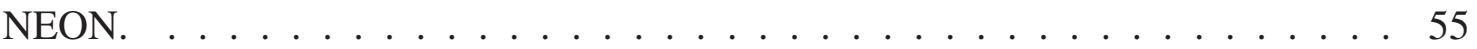

4.6 Decoding polar codes on an NVIDIA Tesla K20c. . . . . . . . . . . . . . . . 60

4.7 Comparison of the power consumption and energy per information bit for the $(2048,1707)$ polar code. . . . . . . . . . . . . . . . 61

4.8 Information throughput and latency of the polar decoders compared with the LDPC decoders of [14] when estimating 524,280 information bits on a Intel Core i7-2600. 65 
5.1 Decoders for a $(1024,512)$ polar code with various initiation interval $\mathcal{I}$ implemented on an FPGA. . . . . . . . . . . . . . . . . . 78

5.2 Decoders for a $(1024,512)$ polar code with various initiation interval $\mathcal{I}$ implemented on an ASIC . . . . . . . . . . . . . . . . . . 78

5.3 Comparison with state-of-the-art polar decoders. . . . . . . . . . . . . . 79

5.4 Comparison with other FPGA implementations. . . . . . . . . . . . . . . 80

5.5 Deeply-pipelined decoders for polar codes of various lengths with rate $R=1 / 2$ implemented on an FPGA. . . . . . . . . . . . . . . . . . . . 81

5.6 Deeply-pipelined decoders for polar codes of various lengths with rate $R=1 / 2$ implemented on an ASIC . . . . . . . . . . . . . . . . . . 81

5.7 Partially-pipelined decoders with initiation interval set to $\mathcal{I}_{\max }$ for polar codes of various lengths with rate $R=5 / 6$ implemented on an FPGA. . . . . . . . . . . . . 82

5.8 Partially-pipelined decoders with initiation interval set to $\mathcal{I}_{\max }$ for polar codes of various lengths with rate $R=5 / 6$ implemented on an ASIC clocked at $1 \mathrm{GHz}$. . . . 82

5.9 Deeply-pipelined decoders for polar codes of length $N=1024$ with common rates implemented on an FPGA. . . . . . . . . . . . . . . . . . . 83

5.10 Deeply-pipelined decoders for polar codes of length $N=1024$ with common rates implemented on an ASIC . . . . . . . . . . . . . . . . . . . 83

6.1 Information throughput and latency for the multi-mode unrolled polar decoders based on the $(2048,1365)$ and $(1024,853)$ master codes, respectively with a $N_{\max }$ of 1024 and $2048 \ldots \ldots \ldots$. . . . . . . . . . . . . . . . . . . 96

6.2 Comparison with state-of-the-art polar decoders. . . . . . . . . . . . . . . . . 97 
I wanna go fast!

Ricky Bobby 



\section{Chapter 1}

\section{Introduction}

Over the last decades we have gradually seen digital circuits take over applications that were traditionally bastions of analog circuits. One of the reasons behind this tendency is our ability to detect and correct errors in digital circuits-circuits making computations with discrete signals as opposed to continuous ones. This ability lead to faster and more reliable communication and storage systems. In some cases it enabled things that we thought might have never been possible e.g. reliable communication with a probe that is located many light years away from our planet.

Right after the second world war, Claude Shannon created a new field-information theoryin which he defined the limit of reliable communications or storage. In his seminal work, Shannon defined what he calls the channel capacity [1], the bound that many researchers have tried to achieve or even approach ever since. Shannon's work does not tell us how this limit can be reached.

While Reed-Solomon (RS) and Bose-Chaudhuri-Hocquenghem (BCH) codes have good errorcorrection performance and are in widespread use even today, it's not until the discovery of turbo codes [2] in the 1990s that error-correcting codes approaching the channel capacity were found. Indeed, while Low-Density Parity-Check (LDPC) codes-initially discovered in the 1960s by Robert Gallager [3] — can also be capacity approaching, their decoding algorithm was too complex for the time and thus were not used until they were independently rediscovered by David McKay in 1997 $[4]$.

The discovery of turbo and LDPC codes, greatly rejuvenated the field of error correction. Often used in conjunction with a RS or a BCH code, standards that feature a turbo or a LDPC code are omnipresent. Nowadays, each home contains at least tens of decoders for these codes. They are used in a plethora of applications such as video broadcasting, wireless and wired communications 
(e.g. WIFI and Ethernet), data storage and more.

The latest findings on the road to achieving channel capacity are polar codes. Invented by Arıkan in 2008 [5] and further refined in 2009 [6], this new class of error-correcting codes, contrary to LDPC and turbo codes, have an explicit—non-random-construction making the implementation of their encoders and decoders simpler than that of LDPC or turbo codes. Polar codes exploit the channel polarization phenomenon by which the probability of correctly estimating codeword bits tends to either 1 (completely reliable) or 0.5 (completely unreliable). These probabilities get closer to their limit as the code length increases when a recursive construction is used. Under the low-complexity Successive-Cancellation (SC) decoding algorithm, polar codes were shown to achieve the symmetric capacity of memoryless channels as their length tends to infinity.

The complexity of the SC algorithm is low but its sequential nature translates in high-latency and low-throughput decoder implementations. To overcome this, new decoding algorithms derived from SC were introduced, most notably [7] and [8]. These algorithms exploit the recursive construction of polar codes along with the a priori knowledge of the code structure. Fast Simplified Successive Cancellation (Fast-SSC), the algorithm described in [8], integrates the Simplified Successive Cancellation (SSC) algorithm described in [7], thus this work builds upon the former.

Fast-SSC represented a significant improvement over the previous algorithms and led to the first hardware decoder achieving a throughput greater than 1 Gbps. However, the optimization presented therein targeted high-rate codes. As low-rate codes are omnipresent in modern wireless communications, it was evident that it would be beneficial to have a closer look at potential improvements for such codes.

In Software-Defined Radio (SDR) applications, researchers and engineers have yet to fully harness the error-correction capability of modern codes. Many are still using classical codes [9], [10] as implementing low-latency high-throughput - exceeding 10 Mbps of information throughputsoftware decoders for turbo or LDPC codes is very challenging. The irregular data access patterns featured in turbo and LDPC decoders make efficient use of Single-Instruction Multiple-Data (SIMD) extensions present on today's processors difficult. To overcome the difficulty of efficiently accessing memory while decoding one frame and still achieve a good throughput, software decoders resorting to inter-frame parallelism (decoding multiple independent frames at the same time) are often proposed [11]-[13]. Inter-frame parallelism comes at the cost of higher latency, as many frames have to be buffered before decoding can be started. Even with a split layer approach to LDPC decoding where intra-frame parallelism can be applied, the latency remains high at multi- 
ple milliseconds on a recent desktop processor [14]. On the other hand, polar codes are well suited for software implementation as their decoding algorithms feature regular memory access patterns.

While the future $5 \mathrm{G}$ standards are still in the works, many documents mention the requirement of peak per-user throughput greater than $10 \mathrm{Gbps}$. Regardless of the algorithm, the state of polar decoder implementations when this research started offered much lower throughput. The fastest SC-based decoder had a throughput of $1.2 \mathrm{Gbps}$ at a clock frequency of $106 \mathrm{MHz}$ [8]. The fastest decoder implementation based on the Belief Propagation (BP) decoding algorithm — an algorithm with higher parallelism than SC-had an average 4.7 Gbps throughput when early termination was used with a clock frequency of $300 \mathrm{MHz}$ [15]. It was evident that a minor improvement over the existing architectures was unlikely to be sufficient to meet the expected throughput requirements of future wireless communication standards.

\subsection{Objectives}

The objectives of this work are to develop polar decoders that (a) have high throughput, low latency and good energy efficiency, (b) are suitable for both hardware and software implementations, and (c) are suitable for use with varying channel conditions. The main objective of this work is to make polar codes more appealing to practical applications.

\subsection{Summary of Thesis Contributions}

This thesis proposes improvements to the state-of-the-art low-complexity decoding algorithm for low-rate polar codes, a code construction alteration method with human-guided criteria, high-speed low-latency software implementations for modern processors, and very-high-speed multi-mode hardware architectures and implementations.

\section{Fast Low-Complexity Hardware Decoders for Low-Rate Polar Codes}

Fast-SSC [8], the state-of-the-art low-complexity decoding algorithm, represents a significant improvement over the previous decoding algorithms. However, the work in [8] and the optimization presented therein targeted high-rate codes. We introduce modifications to the Fast-SSC algorithm to recognize more constituent codes in order to better accommodate low-rate codes and dedicated hardware is added to efficiently decode these new constituent codes. We also propose a code 
construction alteration process to further reduce the latency and increase the throughput. Implementation results using the proposed methods and algorithms are presented. These results show a $22 \%$ to $28 \%$ latency reduction and a $26 \%$ to $34 \%$ throughput improvement with little to negligible coding loss for low-rate moderate-length polar codes.

\section{Low-Latency Software Polar Decoders}

In SDR applications, researchers and engineers have yet to fully harness the error-correction capability of modern codes due to their high computational complexity. The low-complexity encoding and decoding algorithms render polar codes attractive for use in SDR applications where computational resources are limited. We present low-latency software polar decoders that exploit modern processor capabilities. We show how adapting the algorithm at various levels can lead to significant improvements in latency and throughput, yielding polar decoders that are suitable for high-performance SDR applications on modern desktop processors and embedded-platform processors. These proposed decoders have an order of magnitude lower latency and memory footprint compared to state-of-the-art decoders, while maintaining comparable throughput. In addition, we present strategies and results for implementing polar decoders on graphical processing units. Finally, we show that the energy efficiency of the proposed decoders is comparable to state-of-the-art software polar decoders.

\section{Unrolled Hardware Architectures for Polar Decoders}

Conventional polar decoders implement one or a few specialized computational units and reuse them multiple times during the decoding process. We demonstrate that polar decoders can achieve extremely high throughput values and retain moderate complexity. We present a family of architectures for hardware polar decoders using a reduced-complexity successive-cancellation decoding algorithm that employ unrolling. The resulting fully-unrolled architectures are capable of achieving a coded throughput in excess of $400 \mathrm{Gbps}$ and of 1 Tbps on an Field-Programmable Gate-Array (FPGA) or an Application-Specific Integrated Circuit (ASIC), respectively-two to three orders of magnitude greater than current state-of-the-art polar decoders-while maintaining a competitive energy efficiency of $6.9 \mathrm{pJ} / \mathrm{bit}$ on ASIC. Moreover, the proposed architectures are flexible in a way that makes it possible to explore the trade-off between area, throughput and energy efficiency. 


\section{Multi-mode Unrolled Polar Decoding}

Unrolled decoders are architectures that provide the greatest decoding speed, by orders of magnitude compared to their more compact counterparts. However, unrolled decoders are built for a specific, fixed, code i.e. the code length or rate cannot be modified at execution time. This is a major drawback for most modern wireless communication applications that largely benefit from the support of multiple code lengths and rates. We show how an unrolled decoder built specifically for a polar code, of fixed length and rate, can be transformed into a multi-mode decoder supporting many codes of various lengths and rates. More specifically, we show how decoders for moderate-length polar codes contain decoders for many other shorter-yet practical—polar codes of both high and low rates. The required hardware modifications are detailed, and ASIC synthesis and power estimations are provided for the $65 \mathrm{~nm}$ CMOS technology from TSMC. Results show a peak information throughput greater than $20 \mathrm{Gbps}$ either at $250 \mathrm{MHz}$ in $4.29 \mathrm{~mm}^{2}$ or at $500 \mathrm{MHz}$ in $1.71 \mathrm{~mm}^{2}$. Latency is kept under $2 \mu \mathrm{s}$ and $650 \mathrm{~ns}$ for the former and latter.

\subsection{Related Publications}

This doctoral research has resulted in several publications, a partial list of which and how they relate to the chapters of this thesis is provided here.

1. P. Giard, G. Sarkis, C. Thibeault, and W. J. Gross, "A 638 Mbps Low-Complexity Rate 1/2 Polar Decoder on FPGAs,” IEEE Int. Workshop on Signal Process. Syst. (SiPS), Oct. 2015, pp. 1-6. [16]

This conference paper discussed modifications to the Fast-SSC algorithm to recognize more constituent codes in order to better accommodate low-rate codes. Dedicated hardware was presented to efficiently decode these new constituent codes. Also, it proposed to slightly alter the code construction to reduce the latency and increase the throughput at the cost of a small error-correction performance degradation. Results were presented for a 1024-bit polar code with rate $1 / 2$ and for two different FPGAs. The contributions of this paper are included and improved upon in the journal paper below.

2. P. Giard, A. Balatsoukas-Stimming, G. Sarkis, C. Thibeault, and W. J. Gross, "Fast Lowcomplexity Decoders for Low-rate Polar Codes," Springer J. Signal Process. Syst., 2016, invited, to appear. [17] 
This journal publication expended on the conference one by formalizing and improving the code construction alteration process. More FPGA results using the proposed methods, algorithms and implementation were presented. ASIC results along with a comparison against the state-of-the-art ASIC decoder implementations was also provided. The contributions of this paper are discussed in Chapter 3.

3. P. Giard, G. Sarkis, C. Thibeault, and W. J. Gross, "Fast Software Polar Decoders," IEEE Int. Conf. on Acoustics, Speech, and Signal Process. (ICASSP), May 2014, pp. 7555-7559. [18]

This conference paper discussed the decoding of polar codes on modern desktop processors with SIMD instructions. Bottom-up optimization was used to implement the Fast-SSC algorithm taking advantage of the Streaming SIMD Extensions (SSE) and Advanced Vector eXtensions (AVX) of Intel processors. Some of the results of this paper are incorporated in Chapter 4.

4. P. Giard, G. Sarkis, C. Leroux, C. Thibeault, and W. J. Gross, "Low-Latency Software Polar Decoders,” Springer J. Signal Process. Syst., 2016, to appear. [19]

This journal publication expended on the conference one by adapting the decoding algorithm at various levels. It analysed the impact of various strategies on latency and throughput. Results were presented for desktop and embedded-platform processors. Strategies and implementation results were also presented for high-throughput decoder implementations on Graphical Processing Unit (GPU) processors. The contributions of this paper are presented in Chapter 4.

5. P. Giard, G. Sarkis, C. Thibeault, and W. J. Gross, "237 Gbit/s Unrolled Hardware Polar Decoder," IET Electron. Lett., issue 10, vol. 51, pp. 762-763, May 2015. [20]

This journal letter presented a fully-unrolled deeply-pipelined architecture based on the FastSSC decoding algorithm to achieve a throughput greater than 200 Gbps on FPGA. That was two orders of magnitude faster than the state of the art. The architecture presented in this paper is included in Chapter 5.

6. P. Giard, G. Sarkis, C. Thibeault, and W. J. Gross, "Multi-Mode Unrolled Hardware Architectures for Polar Decoders," IEEE Trans. Circuits \& Syst. I, vol. 63, no. 9, pp. 1443-1453, Sep. 2016. [21] 
This journal publication started by expending on the previous one by generalizing the unrolled architecture into a family of architectures offering a flexible trade-off between throughput, area and energy efficiency. More details on the unrolled architecture were given and more results were provided. The example used in the journal letter was significantly improved on all metrics. ASIC results were provided as well as power estimations. These contributions are included in Chapter 5.

This paper also presented a new method to enable the use of multiple code lengths and rates in a fully-unrolled polar decoder architecture. This novel method lead to a length- and rateflexible decoder while retaining the very high speed typical to unrolled decoders. Results were presented for two versions of a multi-mode decoder supporting eight and ten different polar codes, respectively. These contributions are included in Chapter 6.

\subsection{Thesis Organization}

Chapter 2 reviews polar codes, their construction, representations, and encoding and decoding algorithms. It also briefly goes over results for the state-of-the-art decoder implementations from the literature.

In Chapter 3, improvements to the state-of-the-art low-complexity decoding algorithm are presented. A code construction alteration method with human-guided criteria is also proposed. Both aim at reducing the latency and increasing the throughput of decoding low-rate polar codes. The effect on various low-rate moderate-length codes and implementation results are discussed.

Algorithm optimization at various levels leading to low-latency high-throughput decoding of polar codes on modern processors are introduced in Chapter 4. Bottom-up optimization and efficient use of SIMD instructions available on both embedded-platform and desktop processors are proposed in order to parallelize the decoding of a frame, reduce latency and increase throughput. Strategies for efficient implementation of polar decoders on General Purpose GPU (GPGPU) are also presented. Implementation results for all three types of modern processors are discussed.

A family of hardware architectures utilizing unrolling is presented in Chapter 5 showing that polar decoders can achieve extremely high throughput values and retain moderate complexity. Implementations for various rates and code lengths are presented for FPGA and ASIC. The results are compared with the state of the art.

Expending from the previous chapter, Chapter 6 introduces a method to enable the use of 
multiple code lengths and rates in a fully-unrolled polar decoder architecture. This novel method leads to a length- and rate-flexible decoder while retaining the very high speed typical to those decoders. ASIC results are presented for two versions of a multi-mode decoder and compared against the state-of-the-art decoders.

Lastly, conclusions about this thesis are drawn in Chapter 7 and a list of suggested future research topics is presented. 


\section{Chapter 2}

\section{Polar Codes}

\subsection{Construction}

Polar codes exploit the channel polarization phenomenon to achieve the symmetric capacity of a memoryless channel as the code length increases $(N \rightarrow \infty)$. A polarizing construction where $N=2$ is shown in Fig. 2.1a. The probability of correctly estimating bit $u_{1}$ increases compared to when the bits are transmitted without any transformation over the channel $W$. Meanwhile, the probability of correctly estimating bit $u_{0}$ decreases. The polarizing transformation can be combined recursively to create longer codes, as shown in Fig. 2.1b for $N=4$. As the $N \rightarrow \infty$, the probability of successfully estimating each bit approaches either 1 (perfectly reliable) or 0.5 (completely unreliable), and the proportion of reliable bits approaches the symmetric capacity of $W$ [6].

To construct an $(N, k)$ polar code, the $N-k$ least reliable bits, called the frozen bits, are set to zero and the remaining $k$ bits are used to carry information. Fig. 2.2a illustrates non-systematic encoding of an $(8,4)$ polar code, where the frozen bits are indicated in gray and $a_{0}, \ldots, a_{3}$ are the $k=4$ information bits. Encoding is carried out by propagating $\boldsymbol{u}=u_{0}^{7}$ from left to right, through the graph of Fig. 2.2a.

The locations of the information and frozen bits are based on the type and conditions of $W$. Unless specified otherwise, in this thesis we use polar codes constructed according to [22]. The generator matrix, $G_{N}$, for a polar code of length $N$ can be specified recursively so that $G_{N}=F_{N}=$ 


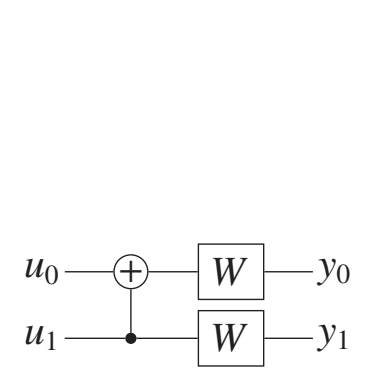

(a) $N=2$

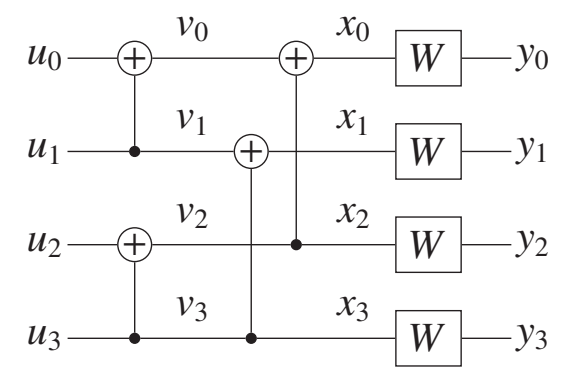

(b) $N=4$

Figure 2.1: Construction of polar codes of lengths 2 and 4 .

$F_{2}^{\otimes \log _{2} N}$, where $F_{2}=\left[\begin{array}{ll}1 & 0 \\ 1 & 1\end{array}\right]$ and ${ }^{\otimes}$ is the Kronecker power. For example, for $N=4, G_{N}$ is

$$
G_{4}=F_{2}^{\otimes 2}=\left[\begin{array}{c|c}
F_{2} & 0 \\
\hline F_{2} & F_{2}
\end{array}\right]=\left[\begin{array}{llll}
1 & 0 & 0 & 0 \\
1 & 1 & 0 & 0 \\
1 & 0 & 1 & 0 \\
1 & 1 & 1 & 1
\end{array}\right] .
$$

In matrix form, non-systematic encoding can be represented as $\boldsymbol{x}=\boldsymbol{u} G_{N}$, where $\boldsymbol{u}$ is a $N$-bit row vector containing the bits to be encoded in the information bit locations. When polar codes were initially proposed, bit-reversed indexing was used. While this changes the bit ordering for both encoding and decoding, the error-correction performance remains unaffected. This change translates into multiplying the generator matrix by the bit-reversal permutation matrix $B_{N}$ [6] (or $\left.\tilde{\Pi}_{N}[5]\right)$, so that $G_{N}=B_{N} F_{N}$. In this thesis, natural indexing is used unless stated otherwise.

\subsection{Tree Representation}

A polar code of length $N$ is the concatenation of two constituent polar codes of length $N / 2$ [6]. Therefore, binary trees are a natural representation of polar codes [7]. Fig. 2.2 illustrates the tree representation of an $(8,4)$ polar code. In Fig. 2.2a, the frozen bits are labeled in gray while the information bits are in black. The corresponding tree, shown in Fig. 2.2b, uses white and black leaf nodes to denote these bits, respectively. The gray nodes of Fig. $2.2 \mathrm{~b}$ correspond to concatenation operations shown in Fig. 2.2a. Moving up in the decoder tree corresponds to the 
concatenation of constituent codes. For example, the concatenation operation circled in blue in Fig. 2.2a corresponds to the node labeled $v$ in Fig. 2.2b.

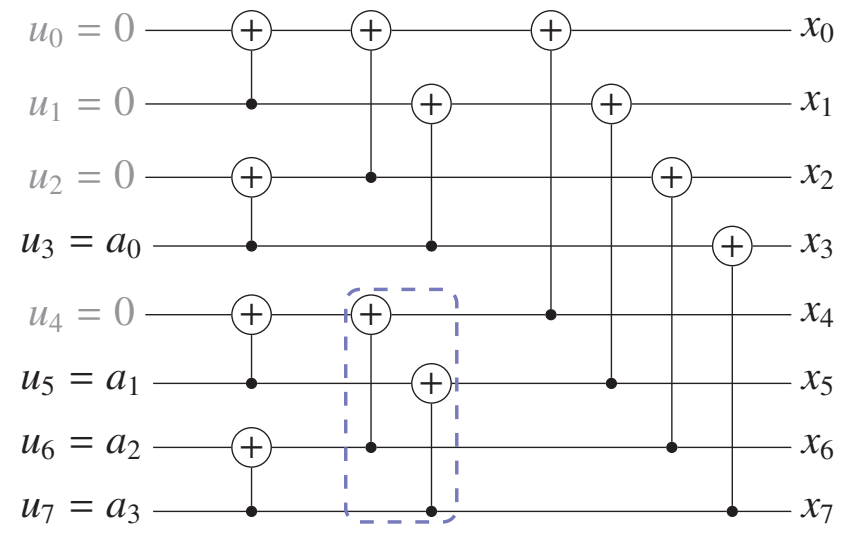

(a) Graph

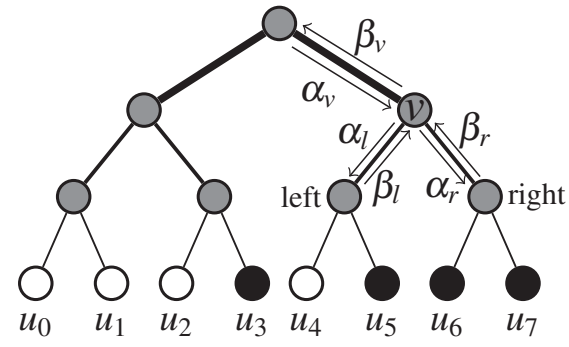

(b) Decoder tree

Figure 2.2: Non-systematic $(8,4)$ polar code represented as a (a) graph and as a (b) decoder tree.

\subsection{Systematic Coding}

Encoding schemes for polar codes can be either non-systematic, as shown in Figs. 2.1b and 2.2a, or systematic as discussed in [23]. Systematic polar codes offer better Bit-Error Rate (BER) than their non-systematic counterparts; while maintaining the same Frame-Error Rate (FER). Furthermore, they allow the use of low-complexity rate-adaptation techniques such as code shortening method proposed in [24]. Flexible low-complexity systematic encoding of polar codes is discussed at length in [25], [26].

Fig. 2.3 shows an example of the low-complexity systematic encoding scheme proposed in [25], [26]. It comprises two non-systematic encoding passes and a bit masking operation in between. For a $(8,4)$ polar code, a $N$-bit vector $\boldsymbol{u}=\left[0,0,0, a_{0}, 0, a_{1}, a_{2}, a_{3}\right]$, where $a_{0}, \ldots, a_{3}$ are the $k=4$ information bits, enters the first non-systematic encoder from the left. Then, using bit masking, the locations corresponding to frozen bits are reset to 0 before propagating the updated vector through the second non-systematic encoder. The end result is a $N$-bit vector $\boldsymbol{x}=\left[p_{0}, p_{1}, p_{2}, a_{0}, p_{3}, a_{1}, a_{2}, a_{3}\right]$, where $p_{0}, \ldots, p_{3}$ are the $N-k=4$ parity bits and $a_{0}, \ldots, a_{3}$ are the $k$ information bits. 


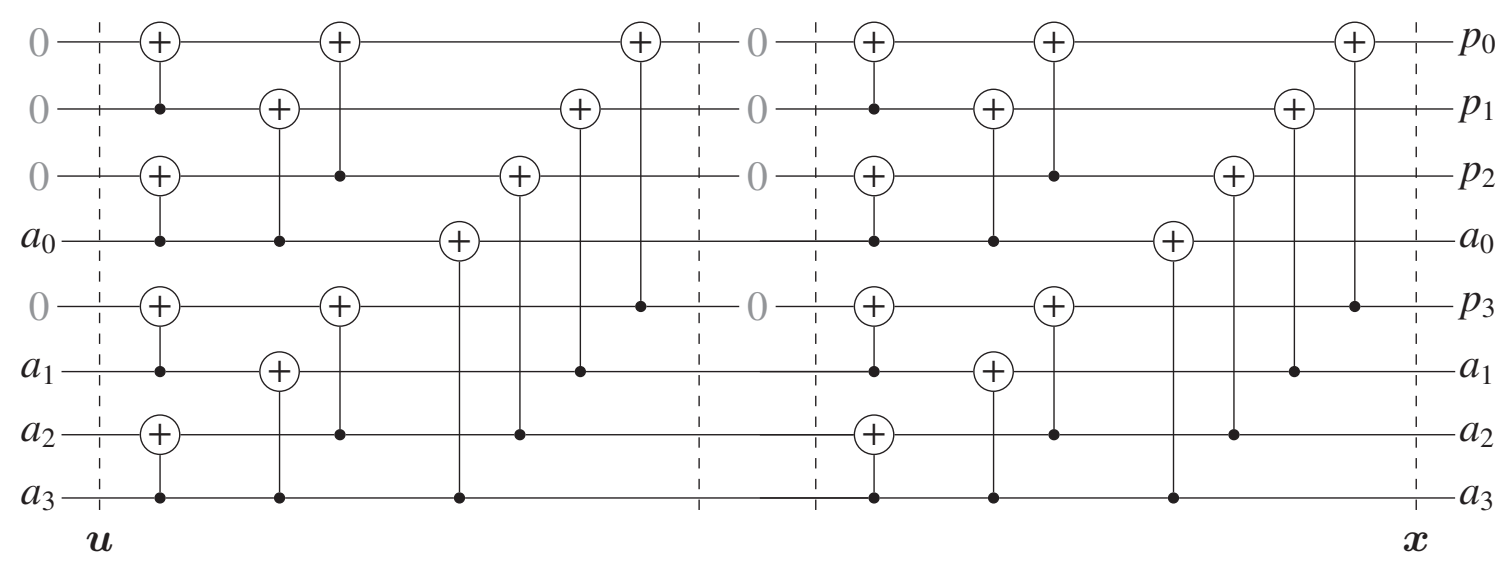

Figure 2.3: Low-complexity systematic encoding of a $(8,4)$ polar code.

This encoding scheme was proven to be correct under certain conditions, conditions that are always met when a construction method leading to polar codes with a good error-correction performance is used e.g. [22]. In this thesis, systematic polar codes are used.

\subsection{Successive-Cancellation Decoding}

In SC decoding, the decoder tree is traversed depth first, selecting left edges before backtracking to right ones, until the size- 1 frozen and information leaf nodes. The messages passed to child nodes are Log-Likelihood Ratios (LLRs); while those passed to parents are bit estimates. These messages are denoted $\alpha$ and $\beta$, respectively. Messages to a left child $l$ are calculated by the $f$ operation using the min-sum algorithm:

$$
\begin{aligned}
\alpha_{l}[i] & =f\left(\alpha_{v}[i], \alpha_{v}\left[i+N_{v} / 2\right]\right) \\
& =\operatorname{sign}\left(\alpha_{v}[i]\right) \operatorname{sign}\left(\alpha_{v}\left[i+N_{v} / 2\right]\right) \min \left(\left|\alpha_{v}[i]\right|,\left|\alpha_{v}\left[i+N_{v} / 2\right]\right|\right),
\end{aligned}
$$

where $N_{v}$ is the size of the corresponding constituent code and $\alpha_{v}$ the LLR input to the node.

Messages to a right child are calculated using the $g$ operation

$$
\begin{aligned}
\alpha_{r}[i] & =g\left(\alpha_{v}[i], \alpha_{v}\left[i+N_{v} / 2\right], \beta_{l}[i]\right) \\
& = \begin{cases}\alpha_{v}\left[i+N_{v} / 2\right]+\alpha_{v}[i], & \text { when } \beta_{l}[i]=0 ; \\
\alpha_{v}\left[i+N_{v} / 2\right]-\alpha_{v}[i], & \text { otherwise, }\end{cases}
\end{aligned}
$$


where $\beta_{l}$ is the bit estimate from the left child.

Bit estimates at the leaf nodes are set to zero for frozen bits and are calculated by performing threshold detection for information ones. After a node has the bit estimates from both its children, they are combined to generate the node's estimate that is passed to its parent

$$
\beta_{v}[i]= \begin{cases}\beta_{l}[i] \oplus \beta_{r}[i], & \text { when } i<N_{v} / 2 \\ \beta_{r}\left[i-N_{v} / 2\right], & \text { otherwise, }\end{cases}
$$

where $\oplus$ is modulo- 2 addition (XOR).

\subsection{Simplified Successive-Cancellation Decoding}

As mentioned above, a polar code is the concatenation of smaller constituent codes. Instead of using the successive-cancellation algorithm on all constituent codes, the location of the frozen bits can be taken into account to use more efficient, lower complexity, algorithms on some of these constituent codes. In [7], decoder tree nodes are split into three categories: Rate-0, Rate-1, and Rate- $R$ nodes.

\subsubsection{Rate-0 Nodes}

Rate- 0 nodes are subtrees whose leaf nodes all correspond to frozen bits. We do not need to use the SC algorithm to decode such a subtree as the exact decision, by definition, is always the all-zero vector.

\subsubsection{Rate-1 Nodes}

These are subtrees where all leaf nodes carry information bits, none are frozen. The maximumlikelihood decoding rule for these nodes is to take a hard decision on the input LLRs:

$$
\beta_{v}[i]= \begin{cases}0, & \text { when } \alpha_{v}[i] \geq 0 \\ 1, & \text { otherwise }\end{cases}
$$

With a fixed-point representation, this operation amounts to copying the most significant bit of the input LLRs. 


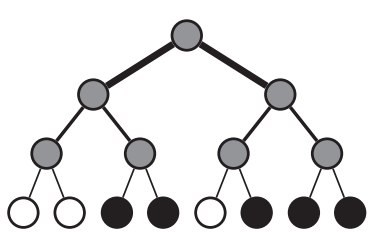

(a) $\mathrm{SC}$

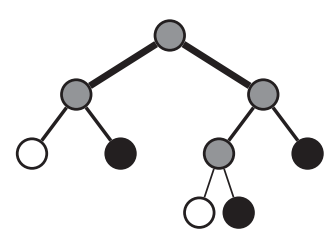

(b) SSC

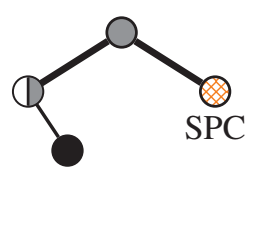

(c) Fast-SSC

Figure 2.4: Decoder trees corresponding to the SC, SSC and Fast-SSC decoding algorithms.

\subsubsection{Rate- $R$ Nodes}

Lastly, Rate- $R$ nodes, where $0<R<1$, are subtrees such that leaf nodes are a mix of information and frozen bits. These nodes are decoded using the conventional SC algorithm until a Rate-0 or Rate-1 node is encountered.

As a result of this categorization, the SSC algorithm trims the SC decoder tree for a $(8,5)$ polar code shown in Fig. 2.4a into the one illustrated in Fig. 2.4b. Rate-1 and Rate-0 nodes are shown in black and white, respectively. Gray nodes represent Rate- $R$ nodes. Trimming the decoder tree leads to a lower decoding latency and an increased decoder throughput.

\subsection{Fast-SSC Decoding}

The Fast-SSC decoding algorithm extends both SC and SSC and further prunes the decoder tree by applying low-complexity decoding rules when encountering certain types of constituent codes.

Three functions- $F, G$ and Combine-are inherited from the original SC algorithm. They correspond to (2.1), (2.2) and (2.3), respectively. Fast-SSC also integrates the decoding algorithms for the Rate- 1 and Rate- 0 nodes of the SSC algorithm.

However, for some Rate- $R$ nodes corresponding to constituent codes with specific frozen-bit locations, a decoding algorithms with lower latency than SC decoding is used. These special cases are:

\subsubsection{Repetition codes}

Repetition codes are constituent codes where only the last bit is an information bit. These codes are efficiently decoded by calculating the sum of the input LLRs and using threshold detection to 
determine the result that is then replicated to form the estimated bits :

$$
\beta_{v}[i]= \begin{cases}0, & \text { when }\left(\sum_{i=0}^{N_{v}-1} \boldsymbol{\alpha}_{v}[i]\right) \geq 0 \\ 1, & \text { otherwise }\end{cases}
$$

where $N_{v}$ is the number of leaf nodes.

\subsubsection{SPC codes}

Single Parity Check (SPC) codes are constituent codes where only the first bit is frozen. The corresponding node is indicated by the cross-hatched orange pattern in Fig. 2.4c. The first step in decoding these codes is to calculate the hard decision of each LLR

$$
\boldsymbol{\beta}_{v}[i]= \begin{cases}0, & \text { when } \boldsymbol{\alpha}_{v}[i] \geq 0 \\ 1, & \text { otherwise }\end{cases}
$$

and then calculating the parity of these decisions

$$
\text { parity }=\bigoplus_{i=0}^{N_{v}-1} \boldsymbol{\beta}_{v}[i]
$$

If the parity constraint is unsatisfied, the estimate of the bit with the smallest LLR magnitude is flipped:

$$
\boldsymbol{\beta}_{v}[i]=\boldsymbol{\beta}_{v}[i] \oplus \text { parity, where } i=\underset{j}{\arg \min }\left(\left|\boldsymbol{\alpha}_{v}[j]\right|\right) \text {. }
$$

\subsubsection{Repetition-SPC codes}

Repetition-SPC codes, or RepSPC codes, are codes whose left constituent code is a repetition code and the right an SPC one. They can be speculatively decoded in hardware by simultaneously decoding the repetition code and two instances of the SPC code: one assuming the output of the repetition code is all 0's and the other all 1's. The correct result is selected once the output of the repetition code is available. This speculative decoding also provides speed gains in software. 


\subsubsection{Other Operations}

The Fast-SSC algorithm introduces other types of operations with the aim of reducing the number of memory accesses, and thus of reducing the latency.

Notably the $G 0 R$ and $C O R$ (or Combine_OR) operations are special cases of the $G$ and Combine operations, respectively (2.2) and (2.3), where the left child is a frozen node i.e. $\beta_{l}$ is known a priori to be the all-zero vector of length $N_{v}$.

Fig. 2.4c shows the tree corresponding to a Fast-SSC decoder.

\subsection{Other SC-based Decoding Algorithms}

Other SC-based algorithms were published where multiple bits are estimated at a time. The next two sections present a brief overview of the most notable ones.

\subsubsection{ML-SSC Decoding}

ML-SSC [27] expands on SSC by using an exhaustive-search maximum-likelihood (ML) decoder to decode rate- $R$ codes once their length and dimension fall below a resource-constrained threshold. The general rule for ML decoding with LLR inputs is given by

$$
\boldsymbol{\beta}_{\boldsymbol{v}}=\underset{\boldsymbol{x} \in C}{\arg \max } \sum_{i}\left(1-2 \boldsymbol{x}_{\boldsymbol{i}}\right) \boldsymbol{\alpha}_{v_{i}}
$$

where $\boldsymbol{\alpha}_{v}$ is the LLR input and $C$ is the list of codewords of the constituent code.

\subsubsection{Hybrid ML-SC Decoding}

The hybrid ML-SC decoding algorithm [28] partitions the polar code graph into $M$ partitions, where each is decoded using an SC decoder until stage $\log _{2} M$ is reached. At that point different rules are used based on the location and count of frozen bits. Instead of conducting an exhaustive search, the ML decoder is simplified by taking advantage of the special structure of polar codes. Nonetheless, no approximations are made and these rules are thus equivalent to the ML decoding rule (2.8).

In the hybrid ML-SC algorithm, SC decoders first produce $M$ LLR values that are used by the following ML decoder section to estimate $M$ bits. These estimated bits are then used to calculate 
the next $M$ LLR values according to (2.2), and so on. Since the progression of the decoding process and the operations applied in hybrid ML-SC are the same as those of ML-SSC, the former can be seen as a special case of the latter.

\subsection{Other Decoding Algorithms}

Besides SC-based algorithms, other algorithms can be used to decode polar codes. On one hand, there are prohibitively complex algorithms, like sphere [29] or linear-programming [30] decoding, practically restricted to short polar codes because of their complexity with regard to code length. On the other hand, there are algorithms that may turn out to be interesting but that did not get much attention yet, in particular the BP and the List-based algorithms. The former is interesting because of its intrinsic high level of parallelism and the latter has great potential because it can significantly improve the error-correction performance of short- to moderate-length polar codes.

\subsubsection{Belief-Propagation Decoding}

The BP algorithm is a well-known algorithm that has been very successfully applied to decode LDPC codes. It was shown in [31] that it can be adapted to decode polar codes as well. BP decoding of a polar code can be seen as applying a flooding decoding schedule to the graph representation of a polar code as opposed to a serial schedule such as the one used in SC-based decoding.

LLRs are iteratively propagated in the graph until a stopping criterion is met. This criterion can either be an early-stopping criterion [32] or simply a fixed maximum number of iterations. Threshold detection is then applied to the resulting LLRs to generate the codeword estimate.

It was shown that BP decoding may require a very large number of iterations to achieve the same error-correction performance as SC. Fig. 2.5 shows an example where BP decoding of a (2048, 1723) polar code requires at least 100 iterations of a flooding schedule to match the performance of SC decoding. At equal error-correction performance, even a fully-parallel BP decoder has a greater latency than an SC decoder.

\subsubsection{List-based Decoding}

In list-based decoding algorithms, several decoding paths are explored using an SC-based algorithm and a constrained list of the $L$-best candidate codewords is built. These $L$-best candidates are 

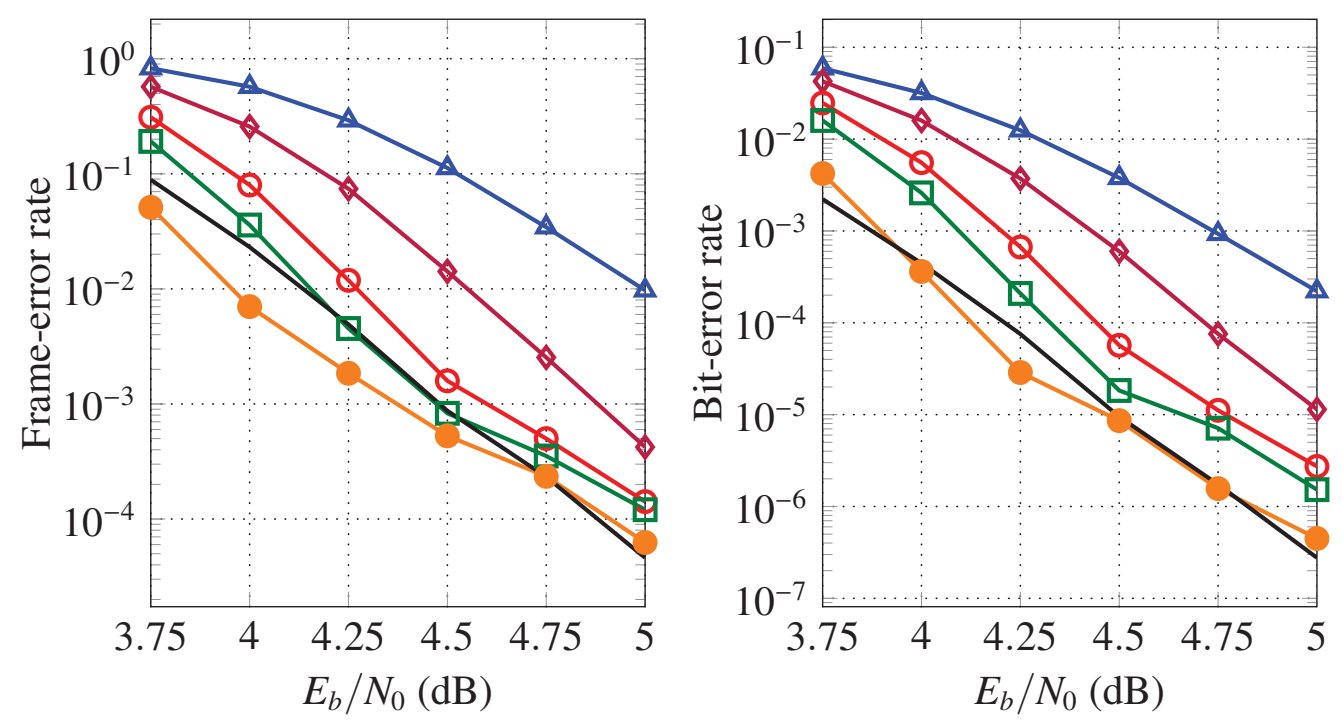

BP: $\neg I=10 \multimap I=20 \multimap I=50 \multimap I=100 \multimap I=1000$

$\mathrm{SC}:-$

Figure 2.5: Error-correction performance of BP and SC decoding for a $(2048,1723)$ polar code, where $I$ is the maximum number of iterations. Data from [26] and used with author's permission.

determined by calculating reliability metric for each of the explored paths. It was shown in [33] that list decoding a polar code concatenated with a Cyclic Redundancy Check (CRC)—List-CRC decoding-greatly improves the error-correction performance over list decoding of a polar code alone. This improvement is significant enough to have polar codes exceed the performance of LDPC codes of similar length and rate.

Fig. 2.6 shows the error-correction performance of List-based decoding of a $(2048,1753)$ polar code. The performance of SC decoding as well as that of the $(1944,1620)$ LDPC code from the 802.11n WIFI standard are included for comparison. A maximum of 10, 20 or 30 iterations of offset min-sum BP decoding with a flooding schedule were used for the LDPC code. All List-CRC decoding curves are for a 16-bit CRC.

In a list-based decoder, the $L$ paths can either be processed in parallel using up to $L$ SC-based decoders or serially by time-multiplexing the use of $M<L$ SC-based decoders. The former results in increased hardware complexity, and the latter in higher latency and lower throughput decoders. Efficient hardware implementations of list-based decoders for polar codes capable of achieving a throughput greater than 5 Gbps was an open problem when we started this thesis and so it remains to this day. 

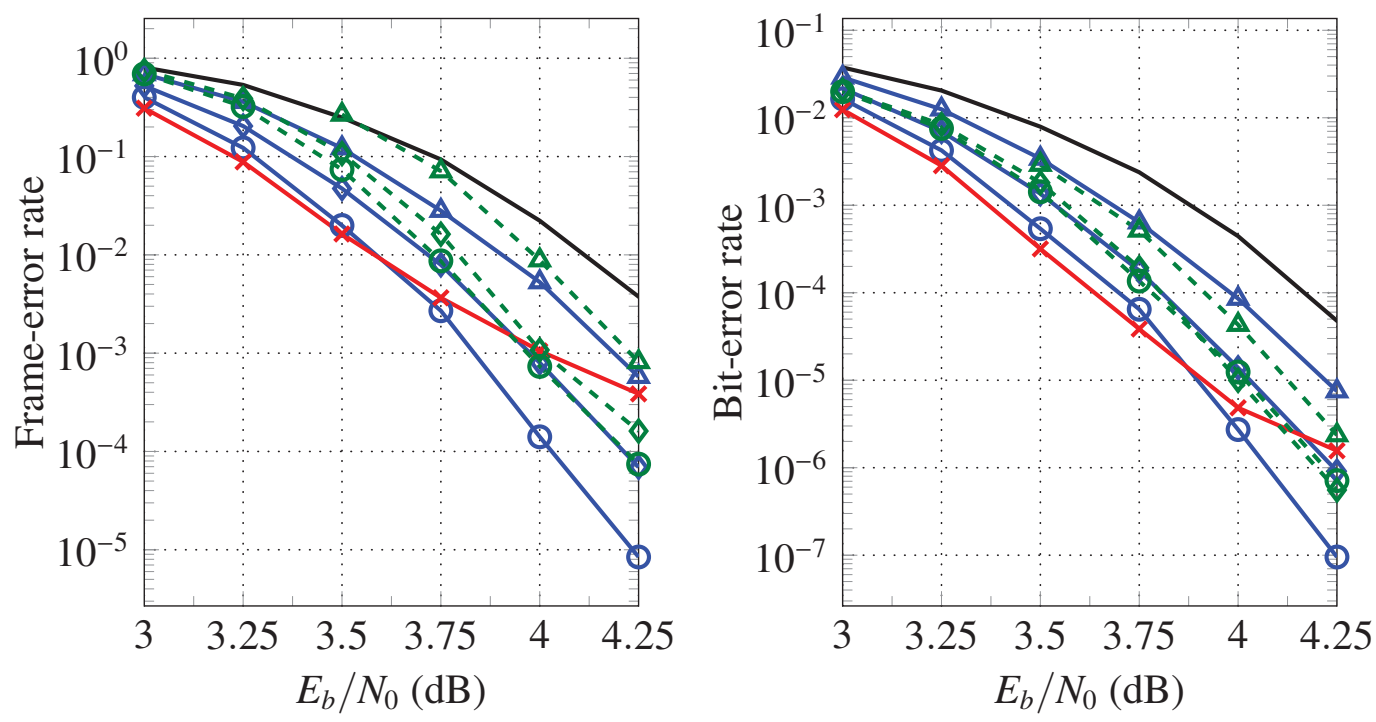

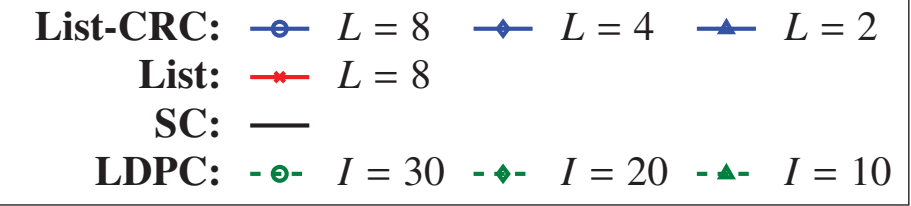

Figure 2.6: Error-correction performance of List, List-CRC and SC decoding of a $(2048,1723)$ polar code versus that of the $(1944,1620) 802.11 n$ LDPC code. $L$ is the maximum number of candidate codewords and $I$ is the maximum number of iterations.

\subsection{SC-based Decoder Hardware Implementations}

Since this thesis proposes SC-based hardware decoders, this section briefly reviews the notable and state-of-the-art SC-based hardware decoders from the literature. But first, a module central to most SC-based decoders is briefly discussed: the Processing Element (PE).

\subsubsection{Processing Element for SC Decoding}

In SC decoding, the soft-value messages are calculated using (2.1) and (2.2). Very early on, a block integrating both calculations was proposed and designated as a PE [34]. Later, it was proposed to use a special PE for the last calculation stage in order to estimate 2 bits simultaneously [35]-[37]. As will be shown below, a different approach was taken in the Fast-SSC implementation. 


\subsubsection{Semi-Parallel Decoder}

The Semi-Parallel Successive-Cancellation (SP-SC) decoder, first proposed in [38] to be later improved in [36], puts a constraint on the number of implemented PEs. It was observed that, in the line SC decoder [39] — which represented the state of the art at the time - , the $N / 2$ PEs were only used twice per decoded frame.

It was shown in [38] that by implementing $P=64$ PEs instead of $N / 2$, a SP-SC decoder would reach $97 \%$ of the speed of a line decoder when decoding a polar code of length $N=2^{11}$, while reducing the hardware complexity by an order of magnitude.

\subsubsection{Two-Phase Decoder}

The Two-Phase Successive-Cancellation (TP-SC) decoder [40] mainly aimed at reducing the memory requirements in a SC-based decoder. As its name suggests, the decoding process is broken down into two parts. Phase 1 and phase 2 are for constituent codes of lengths $N_{v}>\sqrt{N}$ and $N_{v} \leq \sqrt{N}$, respectively.

A pipelined-tree architecture is used to increase the clock frequency and memory is saved by not saving all intermediate LLRs to Random-Access Memory (RAM), instead they are recalculated when then are needed again.

\subsubsection{Processor-like Decoder or the Original Fast-SSC Decoder}

The Fast-SSC implementation of [8] proposed a decoder architecture that contained, at its core, what resembles a processor. As illustrated in Fig. 2.7, the processor featured all the modules required to implement the nodes and operations described in sections 2.4, 2.5 and 2.6.

The decoder could be configured at run time to load a set of instructions corresponding to given polar code. At the time however, the code length was fixed at synthesis time. It was later improved to support any polar code of length $N \leq N_{\max }$ [25].

\subsubsection{Implementation Results}

In this section, results for implementations of the decoders discussed above are presented. All results are for the Altera Stratix IV EP4SGX530KH40C2 FPGA. Table 2.1 shows the resource usage and execution frequency while table 2.2 presents latency and throughput results for a few 


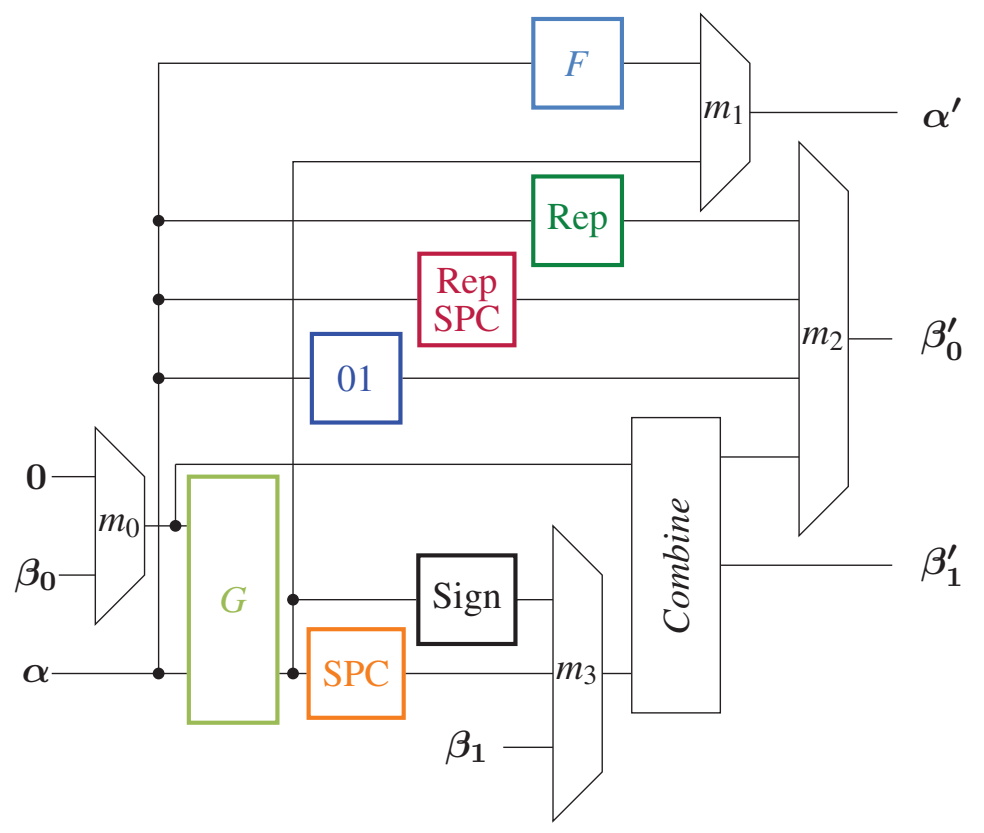

Figure 2.7: Architecture of the data processing unit proposed in [8].

polar codes of various lengths and rates. It should be noted that the TP-SC decoder implementation of [40] lacks the buffers required to sustain its throughput. 
Table 2.1: Post-fitting results for SC-based decoder implementations.

\begin{tabular}{lcrrrc}
\hline Implementation & $N$ & LUTs & Regs. & $\begin{array}{r}\text { RAM } \\
\text { (kbits) }\end{array}$ & $\begin{array}{c}f \\
(\mathrm{MHz})\end{array}$ \\
\hline SP-SC [38] & 1024 & 2,888 & 1,388 & 11.9 & 196 \\
SP-SC [41] & & 2,618 & 1,292 & 13.8 & 169 \\
TP-SC [40] & & 1,940 & 748 & 7.1 & 239 \\
SP-SC [38] & 16,384 & 29,897 & 17,063 & 184.1 & 113 \\
SP-SC [41] & & 2,769 & 1,230 & 206.6 & 168 \\
TP-SC [40] & & 7,815 & 3,006 & 114.6 & 230 \\
Fast-SSC [8] & 25,219 & 6,529 & 285.3 & 106 \\
SP-SC [38] & 32,768 & 58,480 & 33,451 & 364.3 & 66 \\
SP-SC [36], [41] & & 3,263 & 1,304 & 411.6 & 167 \\
Fast-SSC [8] & & 25,866 & 7,209 & 536.1 & 108 \\
\hline
\end{tabular}

Table 2.2: Latency and information throughput for SC-based decoder implementations.

\begin{tabular}{|c|c|c|c|c|}
\hline \multirow[b]{2}{*}{ Implementation } & \multirow{2}{*}{$\begin{array}{l}\text { Code } \\
(N, k)\end{array}$} & \multicolumn{2}{|c|}{ Latency } & \multirow{2}{*}{$\begin{array}{c}\text { Info. T/P } \\
\text { (Mbps) }\end{array}$} \\
\hline & & (CCs) & $(\mu \mathrm{s})$ & \\
\hline SP-SC [38] & $(1024,512)$ & 2,304 & 12 & 44 \\
\hline SP-SC [41] & & 2,604 & 15 & 33 \\
\hline TP-SC [40] & & 2,656 & 11 & 56 \\
\hline SP-SC [38] & $(16384,14746)$ & 34,304 & 304 & 48 \\
\hline SP-SC [41] & & 43,772 & 261 & 57 \\
\hline TP-SC [40] & & 41,600 & 181 & 106 \\
\hline Fast-SSC [8] & & 1,433 & 14 & 1,091 \\
\hline SP-SC [38] & $(32768,29492)$ & 69,120 & 1,047 & 28 \\
\hline SP-SC [36], [41] & & 88,572 & 530 & 56 \\
\hline Fast-SSC [8] & & 2,847 & 26 & 1,081 \\
\hline
\end{tabular}




\section{Chapter 3}

\section{Fast Low-Complexity Hardware Decoders for Low-Rate Polar Codes}

In this chapter, we show how the state-of-the-art low-complexity decoding algorithm can be improved to better accommodate low-rate codes. More constituent codes are recognized in the updated algorithm and dedicated hardware is added to efficiently decode these new constituent codes. We also alter the polar code construction to further decrease the latency and increase the throughput with little to no noticeable effect on error-correction performance. Rate-flexible decoders for polar codes of length 1024 and 2048 are implemented on FPGA and ASIC. Over the previous FPGA work, they are shown to have from $22 \%$ to $28 \%$ lower latency and $26 \%$ to $34 \%$ greater throughput when decoding low-rate codes. On $65 \mathrm{~nm}$ ASIC CMOS technology, the proposed decoder for a $(1024,512)$ polar code is shown to compare favorably against the state-of-the-art ASIC decoders. With a clock frequency of $400 \mathrm{MHz}$ and a supply voltage of $0.8 \mathrm{~V}$, it has a latency of $0.41 \mu$ s and an area efficiency of $1.8 \mathrm{Gbps} / \mathrm{mm}^{2}$ for an energy efficiency of $77 \mathrm{pJ} /$ info. bit. At $600 \mathrm{MHz}$ with a supply of $1 \mathrm{~V}$, the latency is reduced to $0.27 \mu \mathrm{s}$ and the area efficiency increased to $2.7 \mathrm{Gbps} / \mathrm{mm}^{2}$ at $115 \mathrm{pJ} /$ info. bit

\subsection{Introduction}

While the Fast-SSC [8] algorithm represents a significant improvement over the previous decoding algorithms, the work in [8] and the optimization presented therein targeted high-rate codes. In this chapter, we propose modifications of the Fast-SSC algorithm and a code construction alteration 
process targeting low-rate codes. We present results using the proposed methods, algorithms and implementation. These results show a $22 \%$ to $28 \%$ latency reduction and a $22 \%$ to $28 \%$ throughput improvement with little to negligible coding loss for low-rate moderate-length polar codes.

The rest of this chapter is organized as follows. Section 3.2 discusses polar code construction alteration along with our proposed method leading to improved latency and throughput of a hardware decoder. In Section 3.3, modifications to the original Fast-SSC algorithms are proposed in order to further reduce the latency and increase the decoding throughput. Sections 3.4 and 3.5 present the implementation details along with the detailed results on FPGA. Section 3.5 also provides ASIC results for our proposed decoder decoding a $(1024,512)$ polar code for a comparison against state-of-the-art ASIC decoders from the literature. Finally, Section 3.6 concludes this chapter.

\subsection{Altering the Code Construction}

\subsubsection{Original Construction}

As mentioned in Chapter 2, a good polar code is constructed by selecting which bits to freeze, according to the type of channel and its conditions [6], [22], [42], [43]. Fig. 3.1 shows the decoder tree corresponding to the $(1024,512)$ polar code constructed using the technique of [22] where only the node types defined in Table 3.1 are used with the same constraints of [8]. The polar code was optimized for an $E_{b} / N_{0}$ of $2.5 \mathrm{~dB}$. The $F, G, G \_0 R$, Combine and Combine_0R blocks are constrained to a maximum of $P=512$ inputs meaning that, for nodes with a length $N_{v}>P,\left\lceil N_{v} / P\right\rceil$ cycles are required. The Rep, RepSPC and 01 blocks are all executed in one clock cycle. Finally, the SPC-based nodes-OSPC and RSPC - use pipelining and require $\left\lceil N_{v} / P\right\rceil+4$ clock cycles. Thus the decoding latency to decode the tree of Fig. 3.1 using the algorithm and implementation of [8] is 220 Clock Cycles (CCs) and the information throughput is 2.33 bits/CC.

Altering a polar code to further trim the decoder tree can result in a significant latency reduction, without affecting the code rate. By making these modifications however, the error-correction performance is degraded. Although, as will be shown in the next section, the impact can be small, especially if the number of changes is limited. 
Table 3.1: Decoder tree node types supported by the original Fast-SSC polar decoder [8].

\begin{tabular}{ccl}
\hline Name & Color & Description \\
\hline OR & White and gray & Left-half side is frozen. \\
R1 & Gray and black & Right-half side is all information. \\
RSPC & Gray and yellow & Right-half side is an SPC code. \\
OSPC & White and yellow & Left-half side is frozen, right-half side is an SPC code. \\
Rep & Green & Repetition code, maximum length $N_{v}$ of 16. \\
RepSPC & Green and yellow & Concatenation of a repetition code on the left and an SPC code on \\
& & the right, $N_{v}=8$. \\
01 & Black and white & $\begin{array}{l}\text { Fixed-length pattern } N_{v}=4 \text { where the left-half side is frozen and the } \\
\text { right-half side is all information. } \\
\text { rate- } R\end{array}$ \\
& Gray & Mixed rate node. \\
\hline
\end{tabular}

\subsubsection{Altered Polar Code Construction}

In all SC-based decoders, the size of the decoder tree depends on the distribution of frozen and information bit locations in the code. Arikan's original polar code construction only focuses on maximizing the reliability of the information bits. Several altered polar-like code constructions have been proposed in the literature [44]-[46] and their objective is to trade off error-correction performance for decoding complexity reduction by slightly changing the set of information bits, while keeping the code rate fixed. The main idea behind all the altered code constructions is to exchange the locations of a few frozen bits and information bits in order to get more bit patterns that are favorable in terms of decoding latency. In all cases, care must be taken in order to avoid using bit locations that are highly unreliable to transmit information bits.

The method in [44] first defines a small set of bit locations which contains the $n_{s}-h$ least reliable information bit locations along with the $h$ most reliable frozen bit locations. Then, in order to keep the rate fixed, it performs an exhaustive search over all $\left(\begin{array}{c}n_{s} \\ h\end{array}\right)$ possible combinations of the $n_{s}$ elements containing exactly $h$ frozen bit locations and selects the combination that leads to the smallest decoding latency. In [45], the altered construction problem is formalized as a binary integer linear program. Consequently, it is shown that finding the polar code with the lowest decoding complexity under an error-correction performance constraint is an NP-hard problem. For this reason, a greedy approximation algorithm is presented which provides reasonable results at low complexity even for large code lengths. A similar greedy algorithm is presented in [46] for polar codes with more general code lengths of the form $N=l^{n}, l \geq 2$. 


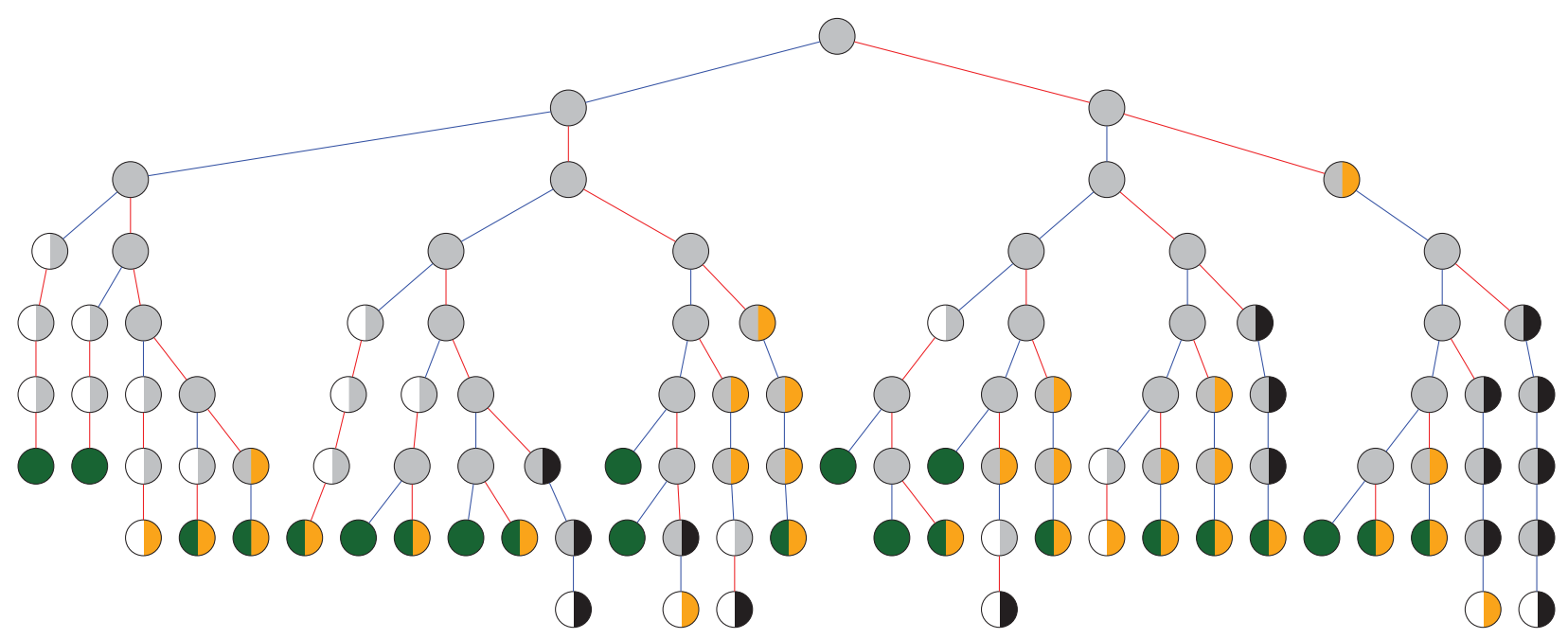

Figure 3.1: Decoder tree for the $(1024,512)$ polar code built using [22] and decoded with the nodes and operations of Table 3.1.

\subsubsection{Proposed Altered Construction}

The methods of [45], [46] only considered rate- 0 and rate- 1 nodes. As such, the results can not be directly applied to Fast-SSC decoding, where several additional types of special nodes exist. For this reason, in this work we follow the more general exhaustive search method of [44], augmented with a human-guided approach.

More specifically, bit-state alterations that would lead to smaller latency are identified by visual inspection of the decoder tree for the unaltered polar code. This list of bit locations is then passed to a program to be added to the bit locations considered by the technique described in [44]. Hence, two lists are composed: one that contains frozen bit locations proposed by the user as well as locations that were almost reliable enough to be used to carry information bits, and one that contains the information bit locations proposed by the user and the locations that barely made it into information bit locations.

The code alteration algorithm then proceeds by gradually calculating the decoding latency for all possible bit swap combinations. A constrained-size and ordered list of the combinations with the lowest decoding latency is kept. Once that list needs to be trimmed, only one entry per latency value is kept by simulating the error-correction performance of the altered code at an $E_{b} / N_{0}$ value of interest. The entry with the best frame-error rate is kept and the others with the same latency are removed from the list. That list containing the best candidates is further trimmed by removing 
all candidates that feature both a greater latency and worse error-correction performance compared to those of their predecessor. Similarly to the technique of [44], our proposed technique does not alter the code rate as the total number of information and frozen bits remains the same.

\section{Human-guided Criteria}

The suggested bits to swap are selected to improve the latency and throughput. Thus, these bit swaps must eliminate constituent codes for which we do not have an efficient decoding algorithm and create ones for which we do. We classify the selection criteria under two categories: the bit swaps that transform frozen bit locations into information bit locations and bit swaps that do the opposite. The former increase the coding rate while the latter reduce it.

In addition to the node type definitions of Table 3.1, the below descriptions of criteria use the following types of subtrees or nodes:

- R1-01: subtree rooted in a R1 node with a 01 leaf node, may contain a chain of R1 nodes

- Rep1: subtree rooted in a R1 node with a leaf Rep node; in Section 3.3, that subtree is made into a node where the left-half side is a repetition code and the right-half side is all information

- R1-RepSPC: subtree rooted in a R1 node with a RepSPC leaf node, may contain a chain of R1 nodes

- Rep-Rep1: subtree where the rate- $R$ node has a left-hand-side and right-hand-side nodes are Rep and Rep1 nodes, respectively

- 0-RepSPC: subtree rooted in a 0R node with a leaf RepSPC node; in Section 3.3, that subtree is made into a node where the left-half side is frozen and the the right-half side is a RepSPC node

Dedicated hardware to efficiently decode Rep1 and 0RepSPC nodes are presented in Section 3.3.

\section{From frozen to information locations:}

1. Unfreezing the second bit of a 01 node that is part of a R1-01 subtree creates an RSPC node. 
2. Changing an RepSPC into an RSPC node by adding the second, third and fifth bit locations.

3. Changing a RSPC node into a R1 node by changing the SPC code into a rate-1 code.

Criterion 1 is especially beneficial where the R1-01 subtree contains a chain of R1 nodes, e.g., Pattern 5 in Fig. 3.2. Similarly, Criterion 2 has a significant impact on R1-RepSPC subtrees containing a chain of R1 nodes, e.g., Pattern 3 in Fig. 3.2.

\section{From information to frozen locations:}

4. Changing a 0R-01 subtree into a Repetition node.

5. Freezing the only information bit location of a Rep node to change it into a rate- 0 code.

6. A specialization of the above, changing a Rep-RepSPC subtree into a 0-RepSPC subtree by changing the left-hand-side Rep node into a rate-0 node.

7. Transforming a Rep-Rep1 subtree into a 0-RepSPC subtree by changing the left-hand-side repetition code into a rate- 0 code and by freezing the fifth bit location of the Rep 1 subtree to change the rate- 1 code into an SPC code.

Consider the decoder tree for a $(512,376)$ polar code as illustrated in Fig. 3.2a, where some frozen bit patterns are circled in blue and numbered for reference. Its implementation results in a decoding latency of 106 clock cycles. That latency can be significantly reduced by freezing information bit locations or by transforming previously frozen locations into information bits.

Notably, five of the bit-swapping criteria-leading to latency reduction-described above are illustrated in Fig. 3.2a. The patterns numbered 1 and 2 are repetition nodes meeting the fourth criterion. Changing both into rate- 0 nodes introduces two new 0R nodes. The patterns 3 to 6 are illustrations of the fourth, second, sixth and first criteria, respectively.

Fig. $3.2 \mathrm{~b}$ shows the resulting decoder tree after the alterations were made. The latency has been reduced from 106 to 82 clock cycles.

\section{Example Results}

Applying our proposed altered construction method, we were able to decrease the decoding latency of the $(1024,512)$ polar code illustrated in Fig. 3.1 from 220 to 189 clock cycles, a 14\% improve- 


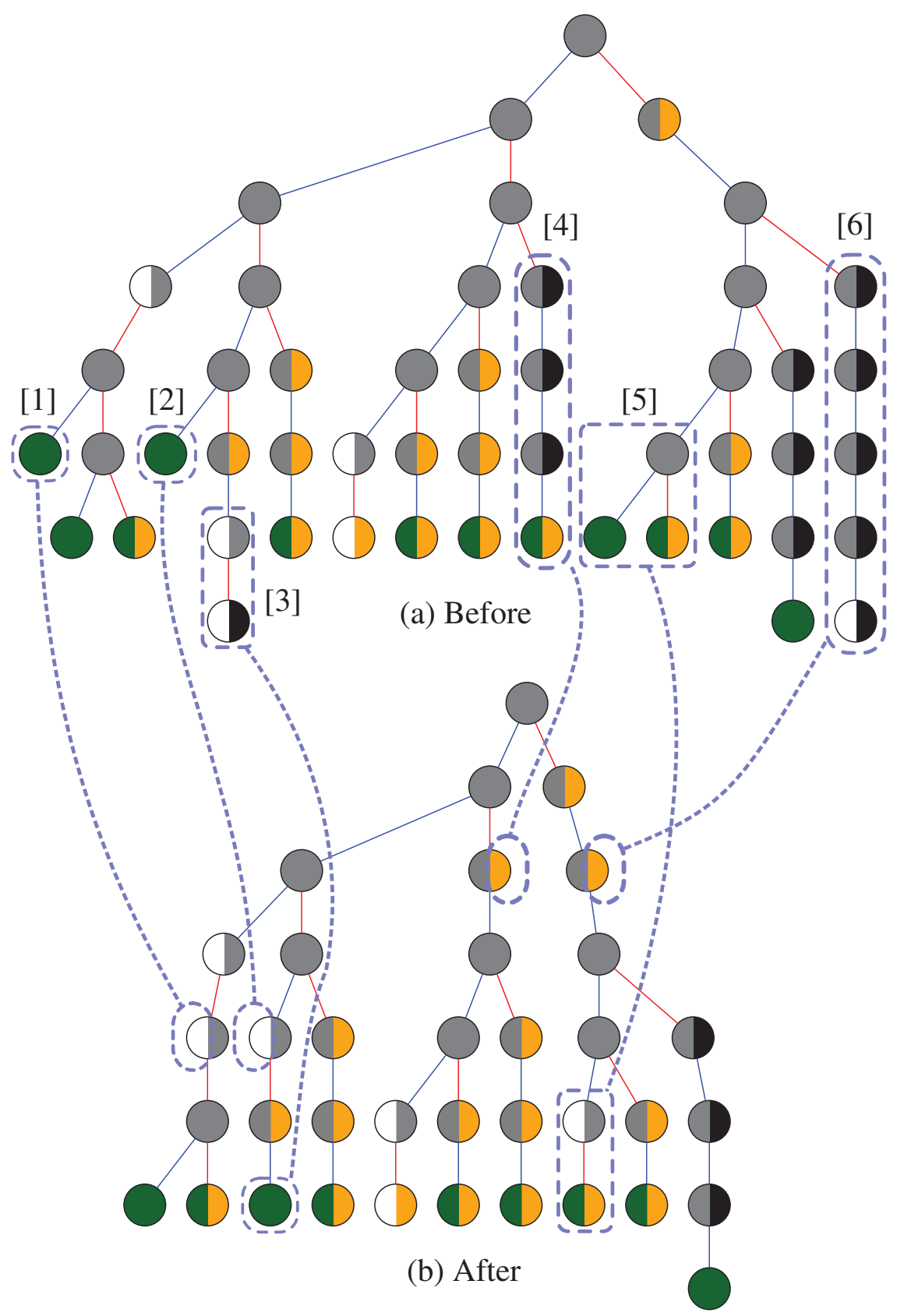

Figure 3.2: Decoder trees for two different $(512,376)$ polar codes, where (a) and (b) are before and after construction alteration, respectively. 


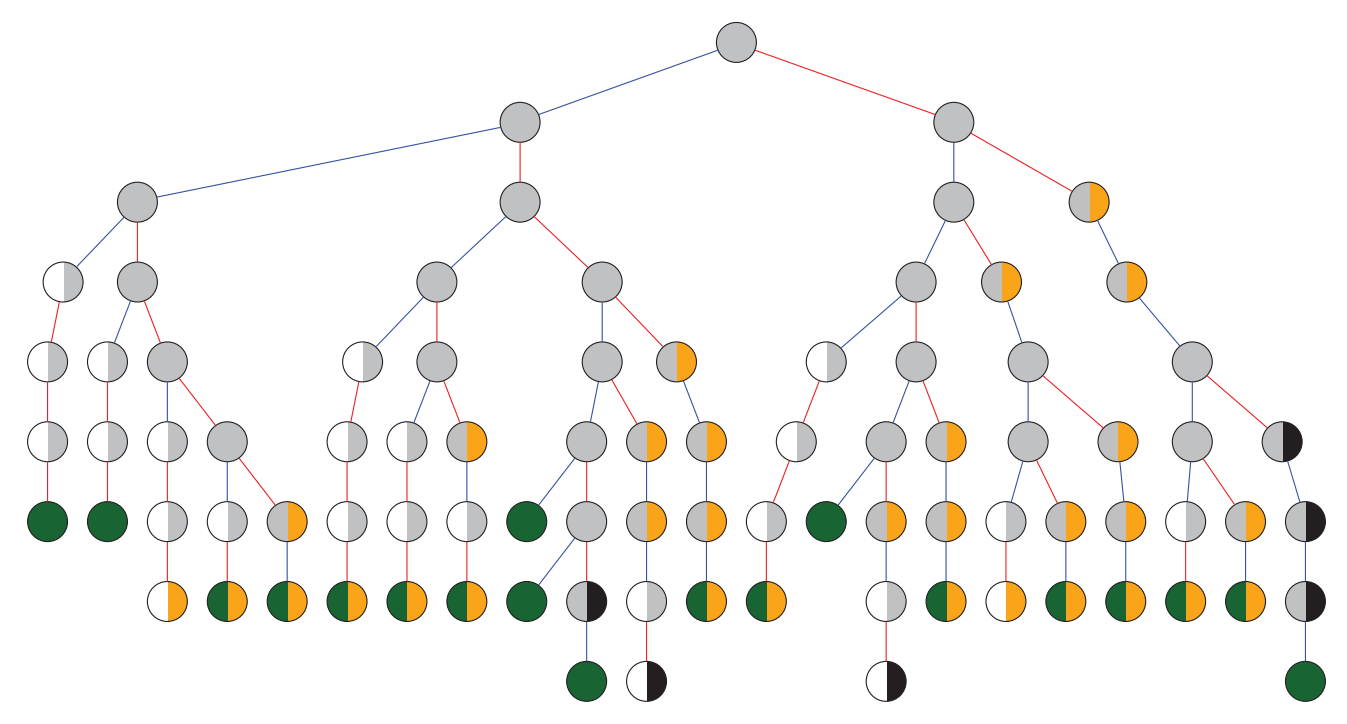

Figure 3.3: Decoder tree for the altered $(1024,512)$ polar code.

ment, with 5 bit swaps. That increases the information throughput to 2.71 bits/CC, up from 2.33 bits/CC. The corresponding decoder tree is shown in Fig. 3.3.

The error-correction performance of the $(1024,512)$ altered code is degraded as illustrated by the markerless black curves in Fig. 3.4. The loss amounts to less than $0.25 \mathrm{~dB}$ at a FER of $10^{-4}$. For wireless applications, which are usually the target for codes of such lengths and rates, this represents the FER range of interest.

Fig. 3.4 also shows the error-correction performance of three other polar codes altered using our proposed method. In the case of these other codes, the alterations have a negligible effect on error-correction performance

\subsection{New Constituent Decoders}

Looking at the decoder tree of Fig. 3.3, it can be seen that some frozen bit patterns occur often. Adding support for more constituent codes to the Fast-SSC algorithm will result in a reduced latency and increased throughput under the constraint that the corresponding computation nodes do not significantly lengthens the critical path of a hardware implementation. As a result of an investigation, the constituent codes of Table 3.2 were added. Furthermore, post-place and route timing analysis showed that the maximum length $N_{v}$ of a Repetition node could be increased from 16 to 32 without affecting the critical path. 

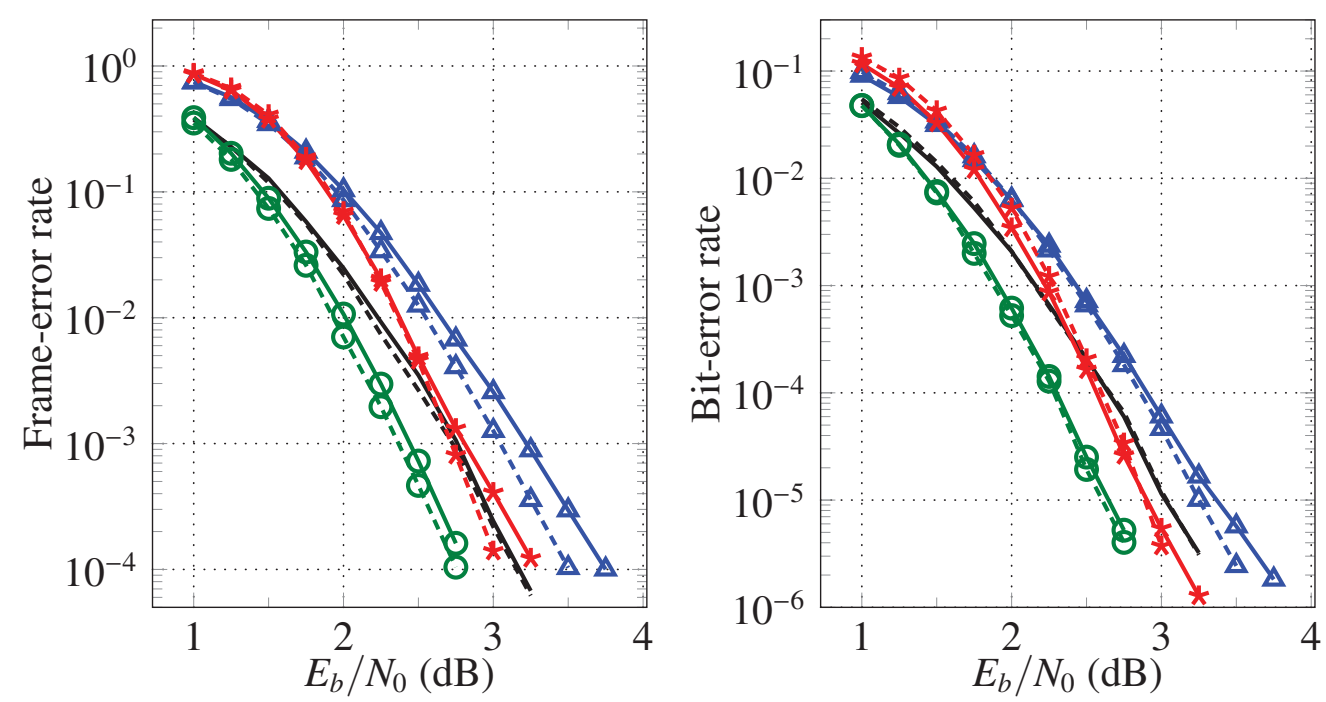

\begin{tabular}{|c|c|}
\hline & \\
\hline 42) & - \\
\hline$-\Delta-(1$ & 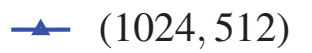 \\
\hline$-a-(2$ & 7 \\
\hline$(2048,1024)$ & $\rightarrow \quad(2048,1024)$ \\
\hline
\end{tabular}

Figure 3.4: Error-correction performance using BPSK over an AWGN channel of the altered codes compared to that of the original codes constructed using the Tal and Vardy method [22].

Table 3.2: New functions performed by the proposed decoder.

\begin{tabular}{|c|c|c|}
\hline Name & Color & Description \\
\hline Rep1 & Green and black & $\begin{array}{l}\text { Repetition code on the left, rate- } 1 \text { code on the right, maxi- } \\
\text { mum length } N_{v} \text { of } 8 \text {. }\end{array}$ \\
\hline $\begin{array}{l}\text { 0RepSPC } \\
001\end{array}$ & $\begin{array}{l}\text { White and lilac } \\
\frac{3}{4} \text { white and } \frac{1}{4} \text { black }\end{array}$ & $\begin{array}{l}\text { Rate- } 0 \text { code on the left, RepSPC code on the right, } N_{v}=16 . \\
\text { Rate- } 0 \text { code on the left, } 01 \text { code on the right, } N_{v}=8 .\end{array}$ \\
\hline
\end{tabular}




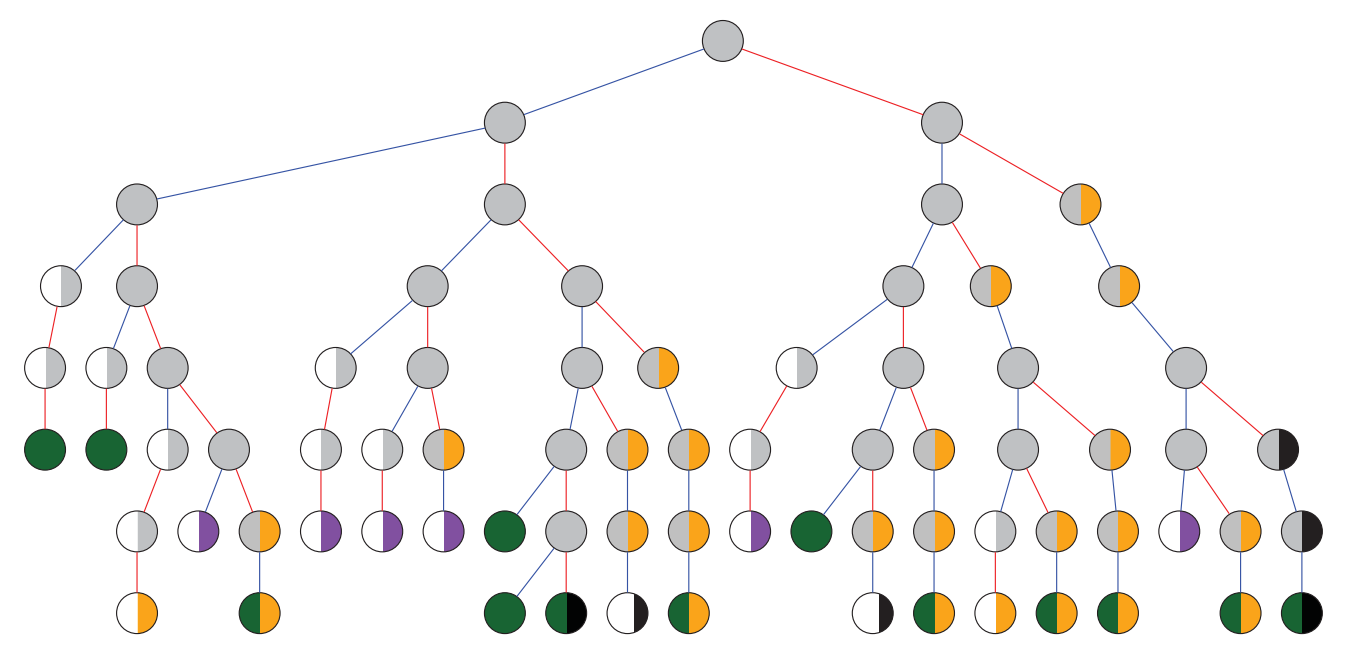

Figure 3.5: Decoder tree for the altered polar code with the added nodes.

The new decoder tree shown in Fig. 3.5 has a decoding latency of 165 clock cycles, a 13\% reduction over the decoder tree of Fig. 3.3 decoded with the original Fast-SSC algorithm. Thus, the information throughput of that polar code has been improved to 3.103 bits/CC.

To summarize, Table 3.3 lists the frozen bit patterns that can be decoded by leaf nodes. It can be seen that the smallest possible leaf node has length $N_{v}=4$ while our proposed decoder tree shown in Fig. 3.5 has a minimum length $N_{v}=8$. In other words, Fig. 3.5 is representative of the patterns listed in Table 3.3 but not comprehensive.

Table 3.3: Frozen bit patterns decoded by leaf nodes.

\begin{tabular}{cl}
\hline Name & Pattern \\
\hline Rep & 0001 \\
& 00000001 \\
& 0000000000000001 \\
& 00000000000000000000000000000001 \\
Rep1 & 00011111 \\
OSPC & 00000111 \\
RepSPC & 00010111 \\
0RepSPC & 0000000000010111 \\
01 & 0011 \\
001 & 00000011 \\
\hline
\end{tabular}




\subsection{Implementation}

\subsubsection{Quantization}

Let $Q_{i}$ be the total number of bits used to represent LLRs internally, $Q_{c}$ be the total number of bits to represent channel LLRs, and $Q_{f}$ be the number of bits among $Q_{i}$ or $Q_{c}$ used to represent the fractional part of any LLR. It was found through simulations that using $Q_{i} \cdot Q_{c} \cdot Q_{f}=6.5 .1$ quantization led to an error-correction performance very close to that of the floating-point number representation as can be seen in Fig. 3.6.
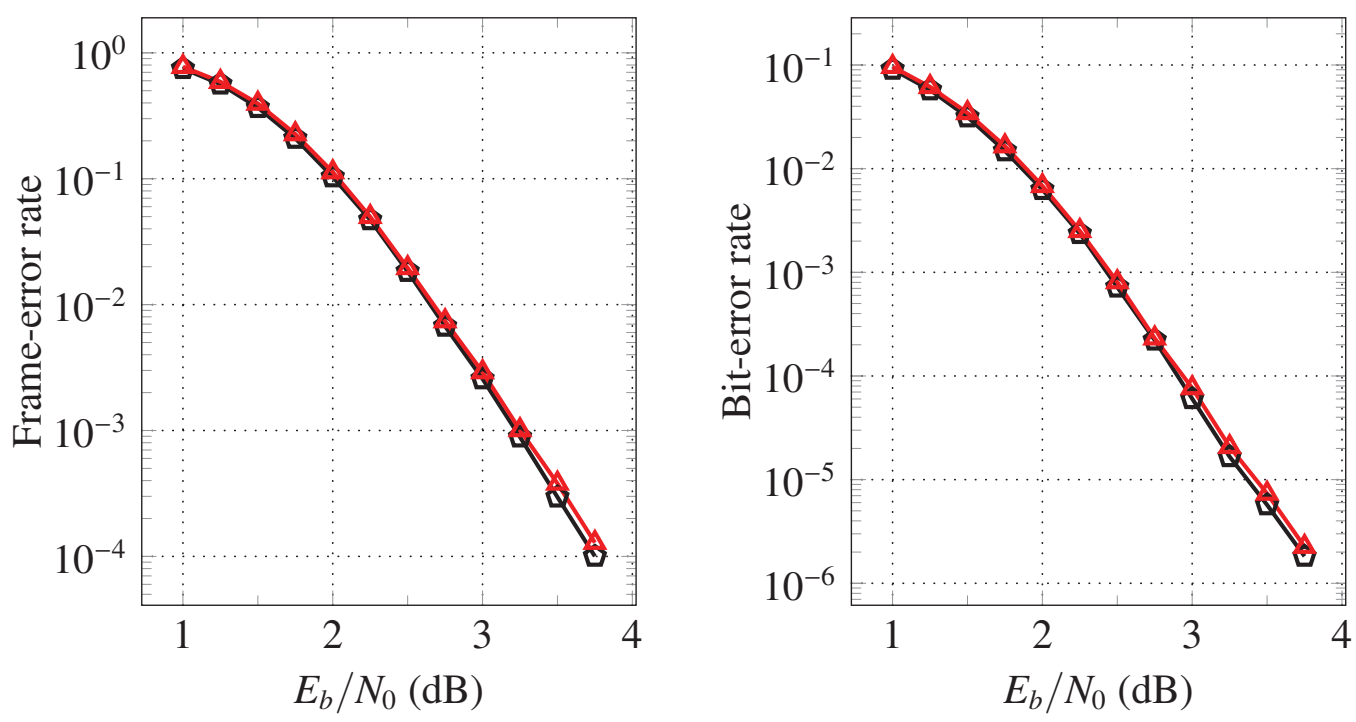

$\rightarrow$ Floating-point $\rightarrow$ Fixed-point 6.5.1

Figure 3.6: Impact of quantization on the error-correction performance of the proposed $(1024,512)$ polar code.

\subsubsection{Rep1 Node}

The Rep1 node decodes Rep1 codes - the concatenation of a repetition code and a rate- 1 code — of length $N_{v}=8$. Its bit-estimate vector $\beta_{0}^{7}$ is calculated using operations described in the previous sections. However, instead of performing the required operations sequentially, the dedicated hardware preemptively calculates intermediate soft values.

Fig. 3.7 shows the architecture of the Rep1 node. It can be seen that there are two $G$ blocks. One preemptively calculates soft values assuming that the Rep block will output $\beta=0$ and the 
other for $\beta=1$. The Rep block provides a single bit estimate corresponding to the information bit the repetition code of length $N_{v}=4$ it is decoding. The outputs of the $G$ blocks go through a Sign block to generate hard decisions. The correct hard decision vector is then selected using the output of the Rep block. Finally, the bit estimate vector $\beta_{0}^{7}$ is built. The highest part, $\beta_{4}^{7}$, is always comprised of the multiplexer output. The lowest part, $\beta_{0}^{3}$, is either a copy of same output or its binary negation. The negated version is selected when the output of the Rep block is 1 .

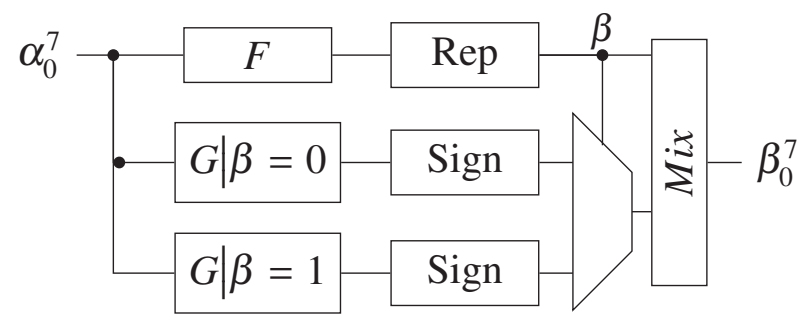

Figure 3.7: Architecture of the Rep1 Node.

Calculations are carried out in one clock cycle. The output of the $F, G$ and Rep blocks are not stored in memory. Only the final result, the bit-estimate vector $\beta_{0}^{7}$, is stored in memory.

\subsubsection{High-Level Architecture}

The high-level architecture of the decoder is presented in Fig. 3.8. Instructions representing the polar decoding operations to be performed are loaded before decoding starts. When the decoder is started, the controller signals the channel loader to start storing channel LLRs, 32 LLRs (160 bits) per clock cycle, into the channel RAM. The controller then starts to execute functions on the processing unit. The processing unit reads LLRs from the Channel or $\alpha$-RAM and writes LLRs to the $\alpha$-RAM. It reads or writes hard decisions to the $\beta$-RAM. The last Combine operation writes the estimated codeword into the Codeword RAM, a memory accessible from outside the decoder.

The decoder is complete with all input and output buffers to accommodate loading a new frame and reading an estimated codeword while a frame is being decoded. The required memory could be made smaller if the nominal throughput required is lower. The loading or outputting of a full frame takes fewer clock cycles than the actual decoding, we have a pipelined operation; under normal operation, the decoder should not be slowed down by the Input/Output (I/O) operations. 


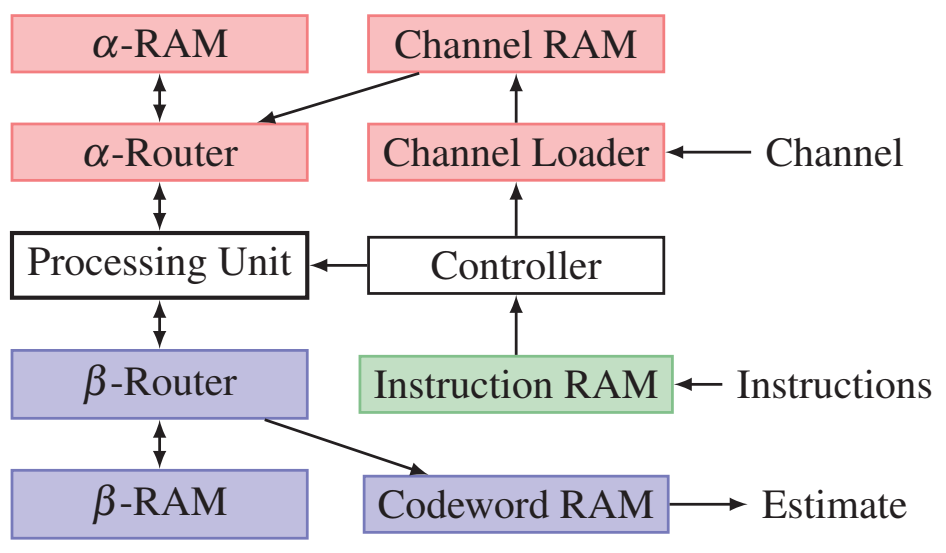

Figure 3.8: High-level architecture of the decoder.

\subsubsection{Processing Unit or Processor}

The core of the decoder is the processing unit illustrated in Fig. 3.9 and based on the Fast-SSC implementation of [8]. Thus, the processing unit features all the modules required to implement the nodes and operations listed in Table 3.1 and described in Section 3.3. Notably, the 01 and RepSPC blocks connected to the $G$ block implement the 001 and 0RepSPC nodes, respectively, where the all-zero vector input is selected at the multiplexer $m_{0}$. The critical path of the decoder corresponds to the ORepSPC node i.e. goes through $G$, RepSPC, the multiplexer $m_{3}$, Combine and the multiplexer $m_{2}$. It is slightly longer than that of [8].

\subsection{Results}

\subsubsection{Verification Methodology}

A software model was used to generate random codewords for transmission using Binary PhaseShift Keying (BPSK) over an Additive White Gaussian-Noise (AWGN) channel. The functionality of the designs was verified both at the Register-Transfer Level (RTL) and at the post-place and route level through simulations. Finally, the same frames were also decoded on an FPGA using an FPGA-in-the-loop setup. For all $E_{b} / N_{0}$ values, a minimum of 100 frames in errors were simulated. 


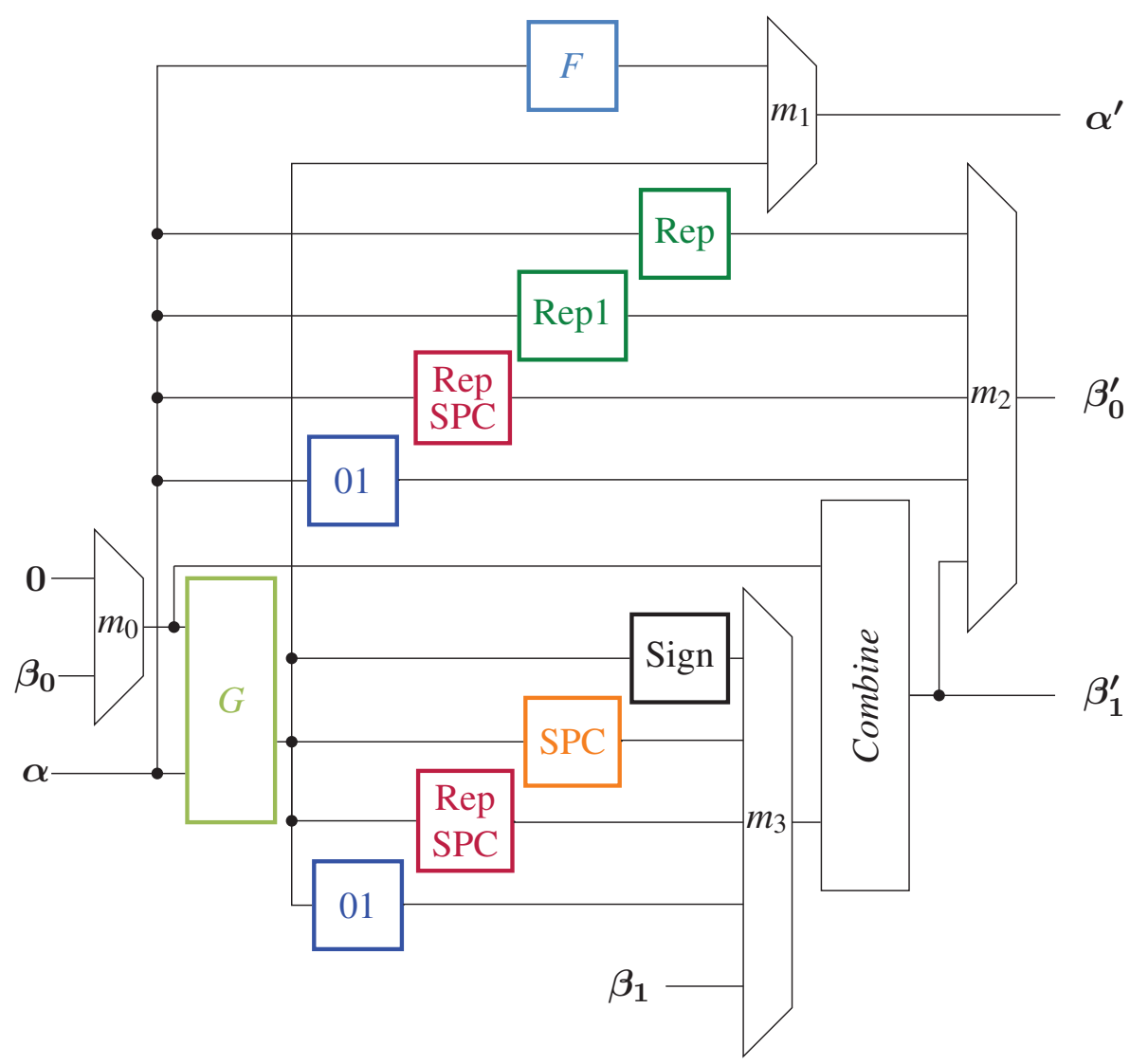

Figure 3.9: Architecture of the processing unit.

\subsubsection{Comparison with State-of-the-art Decoders}

In this section, post-fitting results are presented for the Altera Stratix IV EP4SGX530KH40C2 FPGA. All results are worst-case using the slow $900 \mathrm{mV} 85^{\circ} \mathrm{C}$ timing model. Table 3.4 shows the results for two rate-flexible implementations for polar codes of length 1024 and 2048, respectively. The decoder of [40] is also included for comparison.

Looking at the results for our proposed decoders, it can be observed that the number of LookUp Tables (LUTs) and registers required are very similar for both code lengths. However, the RAM usage differs significantly where decoding a longer code requires more memory as expected. Timing reports show that the critical path corresponds to the ORepSPC node.

Table 3.4 also compares the proposed decoders against the decoder of [40] as well as the original Fast-SSC implementation [8]. The latter was resynthesized so that the decoder only has to accommodate polar codes of length $N=1024$ or $N=2048$ and is marked with an asterisk (*) in 
Table 3.4: Post-fitting results for rate-flexible decoders for moderate-length polar codes.

\begin{tabular}{lrrrrc}
\hline Implementation & $N$ & LUTs & Regs. & $\begin{array}{r}\text { RAM } \\
\text { (kbits) }\end{array}$ & $\begin{array}{c}f \\
(\mathrm{MHz})\end{array}$ \\
\hline$[40]$ & 1024 & 1,940 & 748 & 7.1 & 239 \\
{$[8]^{*}$} & 1024 & 23,020 & 1,024 & 42.8 & 103 \\
& 2048 & 23,319 & 5,923 & 60.9 & 103 \\
this work & 1024 & 23,353 & 5,814 & 43.8 & 103 \\
& 2048 & 23,331 & 5,923 & 61.2 & 103 \\
\hline
\end{tabular}

Table 3.4.

Our work requires at most a $1.4 \%$ increase in used LUTs compared to [8]. The difference in registers can be mostly attributed to register duplication, a measure taken by the fitter to shorten the critical path to meet the requested clock frequency. The Static Random-Access Memory (SRAM) usage was also increased by $2.3 \%$.

Table 3.5 shows the latency and information throughput of the decoders of Table 3.4 when decoding low-rate moderate-length polar codes. It also shows the effect of using a polar codes with altered constructions-as described in Section 3.2-with all Fast-SSC-based decoders. For both [8]* and our work, the results listed as 'altered codes' have the same resource usage and clock frequency as listed in Table 3.4 since these decoders can decode any polar code of length $N=1024$ or $N=2048$ by changing the code description in memory.

Applying the proposed altered construction alone, Table 3.5 shows that decoding these altered codes with the original decoders of [8] results in a $14 \%$ to $21 \%$ latency reduction and a $16 \%$ to $27 \%$ throughput improvement. From the same table, it can be seen that decoding the unaltered codes with the updated hardware decoder integrating the proposed new constituent decoders, the latency is reduced by $4 \%$ to $10 \%$ and the throughput is improved by $4 \%$ to $10 \%$.

Combining the contribution of both the altered construction method and the new dedicated constituent decoders, the proposed work achieves the best latency among all compared decoders. For the polar codes of length $N=1024$, the throughput is 5.7 to 6.1 times greater than that of the two-phase decoder of [40]. Finally, the latency is reduced by $22 \%$ to $28 \%$ and the throughput is increased by $26 \%$ to $34 \%$ over the Fast-SSC decoders of [8].

Table 3.6 presents a comparison of this work against the state-of-the-art ASIC implementations. Our ASIC results are for the $65 \mathrm{~nm}$ CMOS GP technology from TSMC and are obtained with 
Table 3.5: Latency and information throughput comparison for low-rate moderate-length polar codes.

\begin{tabular}{|c|c|c|c|c|}
\hline \multirow{2}{*}{ Implementation } & \multirow{2}{*}{$\begin{array}{l}\text { Code } \\
(N, k)\end{array}$} & \multicolumn{2}{|c|}{ Latency } & \multirow{2}{*}{$\begin{array}{c}\text { Info. T/P } \\
\text { (Mbps) }\end{array}$} \\
\hline & & $(\mathrm{CCs})$ & $\overline{(\mu \mathrm{s})}$ & \\
\hline \multirow[t]{2}{*}{ [40] } & $(1024,342)$ & 2185 & 9.14 & 37 \\
\hline & $(1024,512)$ & 2185 & 9.14 & 56 \\
\hline \multirow[t]{4}{*}[8]{$^{*}$} & $(1024,342)$ & 201 & 1.95 & 175 \\
\hline & $(1024,512)$ & 220 & 2.14 & 240 \\
\hline & $(2048,683)$ & 366 & 3.55 & 192 \\
\hline & $(2048,1024)$ & 389 & 3.78 & 271 \\
\hline \multirow[t]{4}{*}{ altered codes } & $(1024,342)$ & 173 & 1.68 & 204 \\
\hline & $(1024,512)$ & 186 & 1.81 & 284 \\
\hline & $(2048,683)$ & 289 & 2.81 & 243 \\
\hline & $(2048,1024)$ & 336 & 3.26 & 314 \\
\hline \multirow[t]{4}{*}{ this work } & $(1024,342)$ & 193 & 1.87 & 183 \\
\hline & $(1024,512)$ & 204 & 1.98 & 259 \\
\hline & $(2048,683)$ & 334 & 3.24 & 211 \\
\hline & $(2048,1024)$ & 367 & 3.56 & 287 \\
\hline \multirow[t]{4}{*}{ altered codes } & $(1024,342)$ & 157 & 1.52 & 224 \\
\hline & $(1024,512)$ & 165 & 1.60 & 320 \\
\hline & $(2048,683)$ & 274 & 2.66 & 257 \\
\hline & $(2048,1024)$ & 308 & 2.99 & 342 \\
\hline
\end{tabular}

Cadence RTL Compiler. Only registers were used for memory due to the lack of access to an SRAM compiler. Normalized results for the decoders from the literature are also provided. For consistency, only results for a $(1024,512)$ polar code are compared to match what was done in the other works. It should be noted that [15] provides measurement results.

From Table 3.6, it can be seen that both implementations of our proposed decoder-at different supply voltages-are $46 \%$ and $42 \%$ the size of the BP decoder [15] and the combinational decoder [47], respectively, when all areas are normalized to $65 \mathrm{~nm}$ technology. Our work has two orders of magnitude lower latency than the BP decoder of [15], and two to five times lower latency than [37]. The latency of the proposed design is 1.05 times and 0.7 times that of [47], when operating at 400 and $600 \mathrm{MHz}$, respectively. The BP decoder [15] employs early termination and its throughput at $E_{b} / N_{0}=4 \mathrm{~dB}$ is the fastest followed by our proposed design. Since the area reported in [15] excludes 
Table 3.6: Comparison of state-of-the-art ASIC decoders decoding a $(1024,512)$ polar code.

\begin{tabular}{lccccc}
\hline & This work & {$[15]^{\diamond}$} & {$[47]$} & {$[37]$} \\
\hline Algorithm & Fast-SSC & BP & SC & 2-bit SC \\
Technology & $65 \mathrm{~nm}$ & $65 \mathrm{~nm}$ & $90 \mathrm{~nm}$ & $45 \mathrm{~nm}$ \\
Supply $(\mathrm{V})$ & 0.8 & 1.0 & 1.0 & 1.3 & N/A \\
Oper. temp. $\left({ }^{\circ} \mathrm{C}\right)$ & 25 & 25 & $\approx 25$ & N/A & N/A \\
Area $\left(\mathrm{mm}^{2}\right)$ & 0.69 & 0.69 & 1.48 & 3.21 & N/A \\
Area @ 65nm $\left(\mathrm{mm}^{2}\right)$ & 0.69 & 0.69 & 1.48 & 1.68 & 0.4 \\
Frequency $(\mathrm{MHz})$ & 400 & 600 & 300 & 2.5 & 750 \\
Latency $(\mu \mathrm{s})$ & 0.41 & 0.27 & 50 & 0.39 & 1.02 \\
Info. T/P $(\mathrm{Gbps})$ & 1.24 & 1.86 & $2.4 @ 4 \mathrm{~dB}$ & 1.28 & 0.5 \\
Sust. Info. T/P $(\mathrm{Gbps})$ & 1.24 & 1.86 & 1.0 & 1.28 & 0.5 \\
Area Eff. $\left(\mathrm{Gbps} / \mathrm{mm}^{2}\right)$ & 1.8 & 2.7 & $1.6 @ 4 \mathrm{~dB}$ & 0.4 & N/A \\
Power $(\mathrm{mW})$ & 96 & 215 & 478 & 191 & N/A \\
Energy $(\mathrm{pJ} / \mathrm{bit})$ & 77 & 115 & $203 @ 4 \mathrm{~dB}$ & 149 & N/A \\
\hline
\end{tabular}

$\diamond$ Measurement results.

the memory necessary to buffer additional received vectors to sustain the variable decoding latency due to early termination, we also report the sustained throughput for that decoder. The sustained throughput is $1.0 \mathrm{Gbps}$ as a maximum of 15 iterations is required for the $\mathrm{BP}$ decoder to match the error-correction performance of the SC-based decoders. Comparing the information throughput of all decoders- - using the best-case values for BP,- it can be seen that the area efficiency of our decoder is the greatest. Lastly, the power consumption estimations indicate that our decoders are more energy efficient than the BP decoder of [15]. Our proposed decoders are also more energy efficient than that of [47]. However, due to the difference in implementation technology, the results of this latter comparison could change if [47] were to be implemented in $65 \mathrm{~nm}$.

\subsection{Conclusion}

In this chapter, we showed how the original Fast-SSC algorithm implementation could be improved by adding dedicated decoders for three new types of constituent codes frequently appearing in lowrate codes. We also used polar code construction alterations to significantly reduce the latency and increase the throughput of a Fast-SSC decoder at the cost of a small error-correction performance 
loss. Rate-flexible polar decoders for polar codes of lengths 1024 and 2048 were implemented on an FPGA. Four low-rate polar codes with competitive error-correction performance were proposed. Their resulting latency and throughput represent a $22 \%$ to $28 \%$ reduction and a $26 \%$ to $34 \%$ improvement over the previous work, respectively. The information throughput was shown to be $224,320,257$, and $342 \mathrm{Mbps}$ at approximately $100 \mathrm{MHz}$ on the Altera Stratix IV FPGAs for the $(1024,342),(1024,512),(2048,683)$ and $(2048,1024)$ polar codes, respectively. On $65 \mathrm{~nm}$ ASIC CMOS technology, the proposed decoder for a $(1024,512)$ polar code was shown to compare favorably against the state-of-the-art ASIC decoders. With a clock frequency of $400 \mathrm{MHz}$ and a supply voltage of $0.8 \mathrm{~V}$, it has a latency of $0.41 \mu \mathrm{s}$ and an area efficiency of $1.8 \mathrm{Gbps} / \mathrm{mm}^{2}$ for an energy efficiency of $77 \mathrm{pJ} /$ info. bit. At $600 \mathrm{MHz}$ with a supply of $1 \mathrm{~V}$, the latency is reduced to $0.27 \mu \mathrm{s}$ and the area efficiency increased to $2.7 \mathrm{Gbps} / \mathrm{mm}^{2}$ at $115 \mathrm{pJ} /$ info. bit. 


\section{Chapter 4}

\section{Low-Latency Software Polar Decoders}

The low-complexity encoding and decoding algorithms render polar codes attractive for use in SDR applications where computational resources are limited. In this chapter, we present lowlatency software polar decoders that exploit modern processor capabilities. We show how adapting the algorithm at various levels can lead to significant improvements in latency and throughput, yielding polar decoders that are suitable for high-performance SDR applications on modern desktop processors and embedded-platform processors. These proposed decoders have an order of magnitude lower latency and memory footprint compared to state-of-the-art decoders, while maintaining comparable throughput. In addition, we present strategies and results for implementing polar decoders on graphical processing units. Finally, we show that the energy efficiency of the proposed decoders is comparable to state-of-the-art software polar decoders.

\subsection{Introduction}

In SDR applications, researchers and engineers have yet to fully harness the error-correction capability of modern codes due to their high computational complexity. Many are still using classical codes [9], [10] as implementing low-latency high-throughput-exceeding $10 \mathrm{Mbps}$ of information throughput-software decoders for turbo or LDPC codes is very challenging. The irregular data access patterns featured in decoders of modern error-correction codes make efficient use of SIMD extensions present on today's Central Processing Units (CPUs) difficult. To overcome this difficulty and still achieve a good throughput, software decoders resorting to inter-frame parallelism (decoding multiple independent frames at the same time) are often proposed [11]-[13]. Inter-frame 
parallelism comes at the cost of higher latency, as many frames have to be buffered before decoding can be started. Even with a split layer approach to LDPC decoding where intra-frame parallelism can be applied, the latency remains high at multiple milliseconds on a recent desktop processor [14]. This work presents software polar decoders that enable SDR systems to utilize powerful and fast error-correction.

Polar codes provably achieve the symmetric capacity of memoryless channels [6]. Moreover they are well suited for software implementation, due to regular memory access patterns, on both x86 and embedded processors [18], [48], [49]. To achieve higher throughput and lower latency on processors, software polar decoders can also exploit SIMD vector extensions present on today's CPUs. Vectorization can be performed intra-frame [18] or inter-frame [48], [49], with the former having lower decoding latency as it does not require multiple frames to start decoding.

In this work, we explore intra-frame vectorized polar decoders. We propose architectures and optimization strategies that lead to the implementation of high-performance software polar decoders tailored to different processor architectures with decoding latency of $26 \mu$ s for a (32768, 29492) polar code, an order of magnitude performance improvement compared to that of our earlier work [18]. We start Section 4.2 by presenting two different software decoder architectures with varying degrees of specialization. Implementation and results on an embedded processor are discussed in Section 4.3. We also adapt the decoder to suit GPUs, an interesting target for applications where many hundreds of frames have to be decoded simultaneously, and present the results in Section 4.4. Finally, Section 4.5 compares the energy consumption of the different decoders and Section 4.7 concludes this chapter.

This chapter builds upon the work we published in [18] and co-authored in [50]. It provides additional details on the approach as well as more experimental results for modern desktop processors. Both floating- and fixed-point implementations for the final desktop CPU version-the unrolled decoder-were further optimized leading to an information throughput of up to $1.4 \mathrm{Gbps}$. It also adds results for the adaptation of our strategies to an embedded processor leading to a throughput and latency of up to 2.25 and 36 times better, respectively, compared to that of the state-of-the-art software implementation. Compared to the state of the art, both the desktop and embedded processor implementations are shown to have one to two orders of magnitude smaller memory footprint. Lastly, strategies and results for implementing polar decoders on a GPU are presented for the first time. 


\subsection{Implementation on x86 Processors}

In this section we present two different versions of the decoder in terms of increasing design specialization for software; whereas the first version - the instruction-based decoder - takes advantage of the processor architecture it remains configurable at run time and the second one- the unrolled decoder-presents a fully unrolled, branchless decoder fully exploiting SIMD vectorization. In the second version of the decoder, compile-time optimization plays a significant role in the performance improvements. Performance is evaluated for both the instruction-based and unrolled decoders.

It should be noted that, contrary to what is common in hardware implementations e.g. [8], [38], natural indexing is used for all software decoder implementations. While bit-reversed indexing is well-suited for hardware decoders, SIMD instructions operate on independent vectors, not adjacent values within a vector. Using bit-reverse indexing would have mandated data shuffling operations before any vectorized operation is performed.

Both versions, instruction-based decoders and unrolled decoders, use the following functions from the Fast-SSC algorithm [8]: $F, G, G 0 R$, Combine, C0R, Repetition, OSPC, RSPC, RepSPC and P01. An Info function implementing eq. (2.5) is also added.

\section{Methodology for the Experimental Results}

We discuss throughput in information bits per second as well as latency. Our software was compiled using the $\mathrm{C}++$ compiler from GCC 4.9 using the flags "-march=native -funroll-loops -Ofast". Additionally, auto-vectorization is always kept enabled. The decoders are inserted in a digital communication chain to measure their speed and to ensure that optimizations, including those introduced by -0fast, do not affect error-correction performance. In the simulations, we use BPSK over an AWGN channel with random codewords.

The throughput is calculated using the time required to decode a frame averaged over 10 runs of 50,000 and 10,000 frames each for the $N=2048$ and the $N>2048$ codes, respectively. The time required to decode a frame, or latency, also includes the time required to copy a frame to decoder memory and copy back the estimated codeword. Time is measured using the high precision clock provided by the Boost Chrono library.

In this work we focus on decoders running on one processor core only since the targeted application is SDR. Typically, an SDR system cannot afford to dedicate more than a single core to 
error-correction as it has to perform other functions simultaneously. For example, in SDR implementations of long term evolution (LTE) receivers, the orthogonal frequency-division multiplexing (OFDM) demodulation alone is approximately an order of magnitude more computationally demanding than the error-correction decoder [9], [10], [51].

\subsubsection{Instruction-based Decoder}

The Fast-SSC decoder implemented on a FPGA in [8] closely resembles a CPU with wide SIMD vector units and wide data buses. Therefore, it was natural to use a similar design for a software decoder-Fast-SSC instructions are parsed from a text file-, leveraging SIMD instructions. This section describes how the algorithm was adapted for a software implementation. As fixed-point arithmetic can be used, the effect of quantization is shown.

\section{Using Fixed-Point Numbers}

On processors, fixed-point numbers are represented with at least 8 bits. As illustrated in Fig. 4.1, using 8 bits of quantization for LLRs results in a negligible degradation of error-correction performance over a floating-point representation. At a FER of $10^{-8}$ the performance loss compared to a floating-point implementation is less than $0.025 \mathrm{~dB}$ for the $(32768,27568)$ polar code. With custom hardware, it was shown in [8] that 6 bits are sufficient for that polar code. It should be noted that in Fast-SSC decoding, only the G function adds to the amplitude of LLRs and it is carried out with saturating adders.

With instructions that can work on registers of packed 8-bit integers, the SIMD extensions available on most general-purpose x 86 and ARM processors are a good fit to implement a polar decoder.

\section{Vectorizing the Decoding of Constituent Codes}

On x86-64 processors, the vector instructions added with SSE support logic and arithmetic operations on vectors containing either 4 single-precision floating-point numbers or 16 8-bit integers. Additionally, x86-64 processors with AVX instructions can operate on data sets of twice that size. Below are the operations benefiting the most from explicit vectorization.

$F$ : the $f$ operation (2.1) is often executed on large vectors of LLRs to prepare values for other processing nodes. The min() operation and the sign calculation and assignment are all vectorized. 

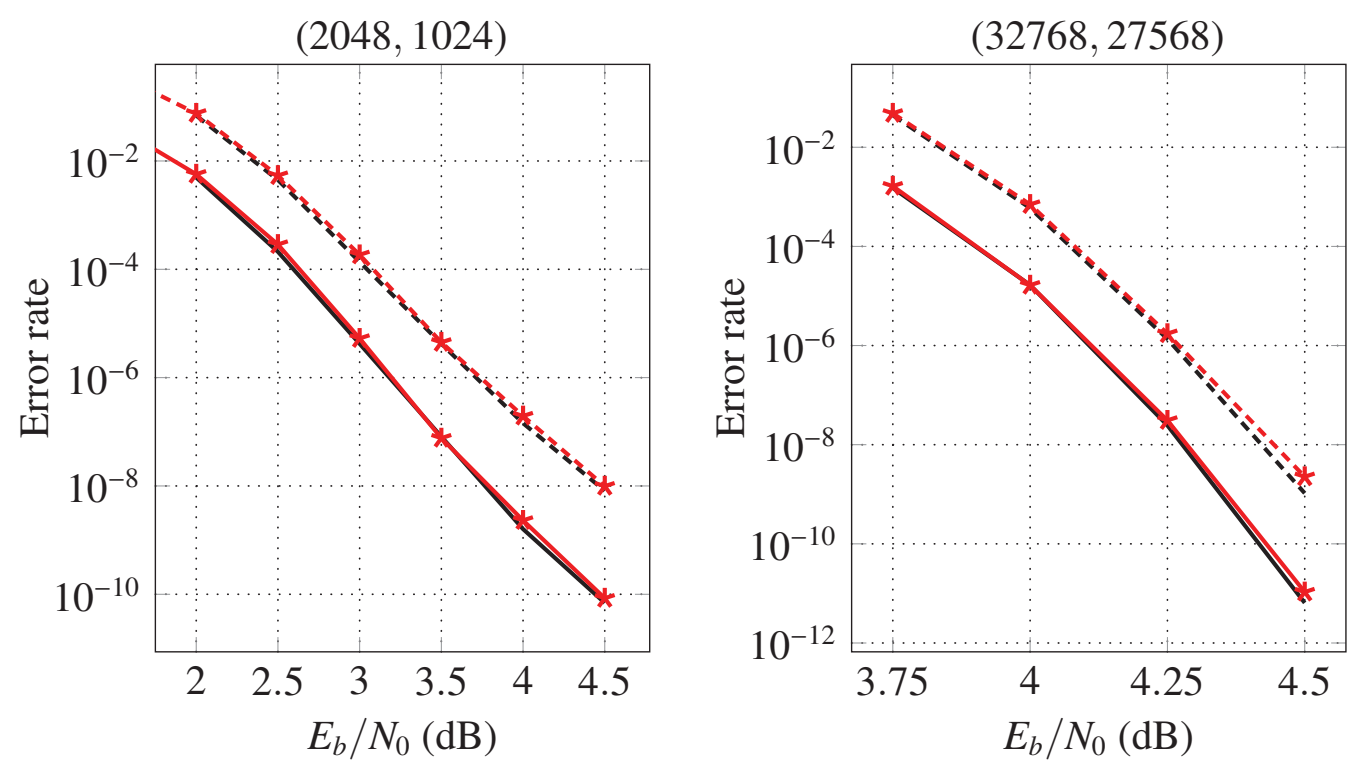

BER: — Floating-point $\rightarrow$ 8-bit fixed-point

FER: --.- Floating-point - *- 8-bit fixed-point

Figure 4.1: Effect of quantization on error-correction performance.

$G$ and $G 0 R$ : the $g$ operation is also frequently executed on large vectors. Both possibilities, the sum and the difference, of (2.2) are calculated and are blended together with a mask to build the result. The $G 0 R$ operation replaces the G operation when the left hand side of the tree is the all-zero vector.

Combine and COR: the Combine operation combines two estimated bit-vectors using an XOR operation in a vectorized manner. The $C O R$ operation is to Combine what $G 0 R$ is to G.

SPC decoding: locating the LLR with the minimum magnitude is accelerated using SIMD instructions.

\section{Data Representation}

For the decoders using floating-point numbers, the representation of $\beta$ is changed to accelerate the execution of the $g$ operation on large vectors. Thus, when floating-point LLRs are used, $\beta_{l}[i] \epsilon$ $\{+1,-1\}$ instead of $\{0,1\}$. As a result, (2.2) can be rewritten as

$$
g\left(\alpha_{v}[i], \alpha_{v}\left[i+N_{v} / 2\right], \beta_{l}[i]\right)=\alpha_{v}[i] * \beta_{l}[i]+\alpha_{v}\left[i+N_{v} / 2\right] .
$$


This removes the conditional assignment and turns $g()$ into a multiply-accumulate operation, which can be performed efficiently in a vectorized manner on modern CPUs. For integer LLRs, multiplications cannot be carried out on 8-bit integers. Thus, both possibilities of (2.2) are calculated and are blended together with a mask to build the result. The Combine operation is modified accordingly for the floating-point decoder and is computed using a multiplication with $\beta_{l}[i] \in\{+1,-1\}$.

\section{Architecture-specific Optimizations}

The decoders take advantage of the SSSE 3, SSE 4.1 and AVX instructions when available. Notably, the sign and abs instructions from SSSE 3 and the blendv instruction from SSE 4.1 are used. AVX, with instructions operating on vectors of 256 bits instead of the 128 bits, is only used for the floating-point implementation since it does not support integer operations. Data was aligned to the 128 (SSE) or 256-bit (AVX) boundaries for faster accesses.

\section{Implementation Comparison}

Here we compare the performance of three implementations. First, a non-explicitly vectorized version $^{1}$ using floating-point numbers. Second an explicitly vectorized version using floatingpoint numbers. Third, the explicitly vectorized version using a fixed-point number representation. In Table 4.1, they are denoted as Float, SIMD-Float and SIMD-int8 respectively.

Results for decoders using the floating-point number representation are included as the efficient implementation makes the resulting throughput high enough for some applications. The decoders ran on a single core of an Intel Core i7-4770S clocked at $3.1 \mathrm{GHz}$ with Turbo disabled.

Comparing the throughput and latency of the Float and SIMD-Float implementations in Table 4.1 confirms the benefits of explicit vectorization in this decoder. The performance of the SIMD-Float implementation is only $21 \%$ to $38 \%$ slower than the SIMD-int8 implementation. This is not a surprising result considering that the SIMD-Float implementation uses the AVX instructions operating on vectors of 256 bits while the SIMD-int8 version is limited to vectors of 128 bits. Table 4.1 also shows that vectorized implementations have 3.6 to 5.8 times lower latency than the floating-point decoder.

\footnotetext{
${ }^{1}$ As stated above, compiler auto-vectorization is always kept enabled.
} 
Table 4.1: Decoding polar codes with the instruction-based decoder.

\begin{tabular}{cccc}
\hline $\begin{array}{c}\text { Code } \\
(N, k)\end{array}$ & Implementation & $\begin{array}{c}\text { Info T/P } \\
(\mathrm{Mbps})\end{array}$ & $\begin{array}{c}\text { Latency } \\
(\mu \mathrm{s})\end{array}$ \\
\hline$(2048,1024)$ & Float & 20.8 & 49 \\
& SIMD-Float & 75.6 & 14 \\
& SIMD-int8 & 121.7 & 8 \\
$(2048,1707)$ & Float & 41.5 & 41 \\
& SIMD-Float & 173.9 & 10 \\
& SIMD-int8 & 209.9 & 8 \\
$(32768,27568)$ & Float & 32.4 & 825 \\
& SIMD-Float & 124.3 & 222 \\
& SIMD-int8 & 175.1 & 157 \\
$(32768,29492)$ & Float & 40.8 & 723 \\
& SIMD-Float & 160.1 & 184 \\
& SIMD-int8 & 198.6 & 149 \\
\hline
\end{tabular}

\subsubsection{Unrolled Decoder}

The goal of this design is to increase vectorization and inlining and reduce branches in the resulting decoder by maximizing the information specified at compile-time. It also gets rid of the indirections that were required to get good performance out of the instruction-based decoder.

\section{Generating an Unrolled Decoder}

The polar codes decoded by the instruction-based decoders presented in Section 4.2.1 can be specified at run-time. This flexibility comes at the cost of increased branches in the code due to conditionals, indirections and loops. Creating a decoder dedicated to only one polar code enables the generation of a branchless fully-unrolled decoder. In other words, knowing in advance the dimensions of the polar code and the frozen bit locations removes the need for most of the control logic and eliminates branches there.

A tool was built to generate a list of function calls corresponding to the decoder tree traversal. It was first described in [50] and has been significantly improved since its initial publication notably to add support for other node types as well as to add support for GPU code generation. Listing 1 shows an example decoder that corresponds to the $(8,5)$ polar code whose dataflow graph is shown 


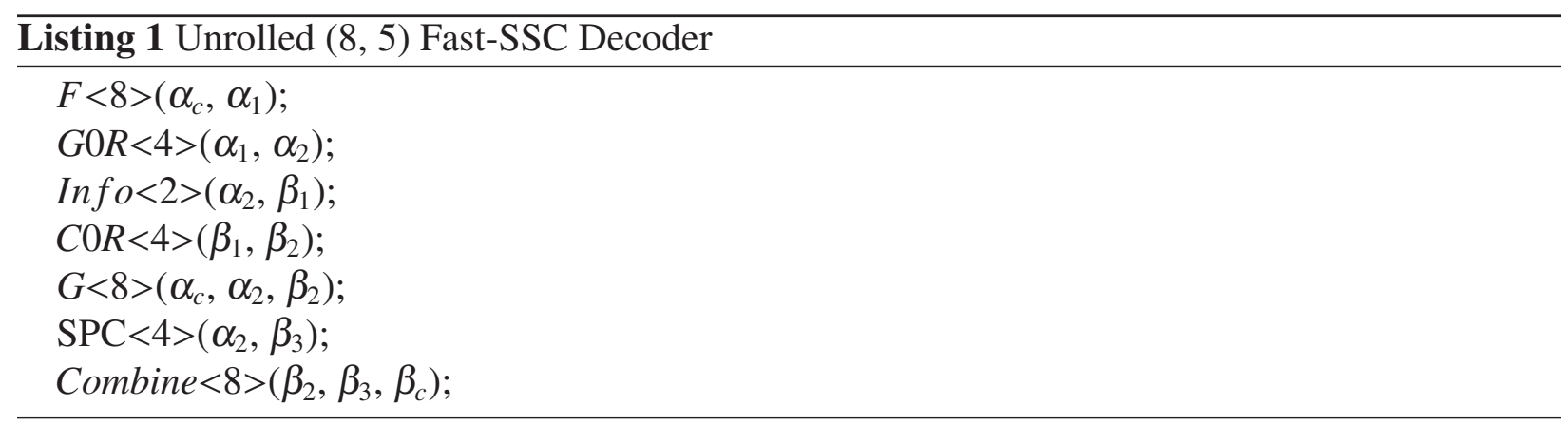

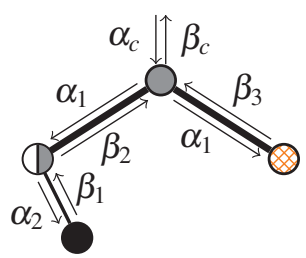

(a) Messages

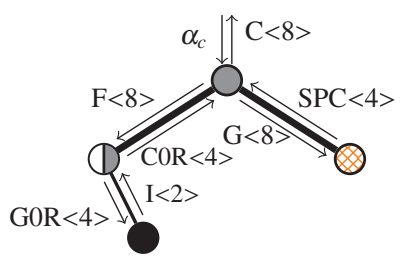

(b) Operations

Figure 4.2: Dataflow graph of a $(8,5)$ polar decoder.

in Fig. 4.2. For brevity and clarity, in Fig. 4.2b, I corresponds to the Info function.

\section{Eliminating Superfluous Operations on $\beta$-Values}

Every non-leaf node in the decoder performs the combine operation (2.3), rendering it the most common operation. In (2.3), half the $\beta$ values are copied unchanged to $\beta_{v}$. One method to significantly reduce decoding latency is to eliminate those superfluous copy operations by choosing an appropriate layout for $\beta$ values in memory: Only $N \beta$ values are stored in a contiguous array aligned to the SIMD vector size. When a combine operation is performed, only those values corresponding to $\beta_{l}$ will be updated. Since the stage sizes are all powers of two, stages of sizes equal to or larger than the SIMD vector size will be implicitly aligned so that operations on them are vectorized.

\section{Improved Layout of the $\alpha$-memory}

Unlike in the case of $\beta$ values, the operations producing $\alpha$ values, $f$ and $g$ operations, do not copy data unchanged. Therefore, it is important to maximize the number of vectorized operations to increase decoding speed. To this end, contiguous memory is allocated for the $\log _{2} N$ stages of the 
decoder. The overall memory and each stage is aligned to 16 or 32-byte boundaries when SSE or AVX instructions are used, respectively. As such, it becomes possible to also vectorize stages smaller than the SIMD vector size. The memory overhead due to not tightly packing the stages of $\alpha$ memory is negligible. As an example, for an $N=32,768$ floating-point polar decoder using AVX instructions, the size of the $\alpha$ memory required by the proposed scheme is 262,208 bytes, including a 60-byte overhead.

\section{Compile-time Specialization}

Since the sizes of the constituent codes are known at compile time, they are provided as template parameters to the functions as illustrated in Listing 1. Each function has two or three implementations. One is for stages smaller than the SIMD vector width where vectorization is not possible or straightforward. A second one is for stages that are equal or wider than the largest vectorization instruction set available. Finally, a third one provides SSE vectorization in an AVX or AVX2 decoder for stages that can be vectorized by the former, but are too small to be vectorized using AVX or AVX2. The last specialization was noted to improve decoding speed in spite of the switch between the two SIMD extension types.

Furthermore, since the bounds of loops are compile-time constants, the compiler is able to unroll loops where it sees fit, eliminating the remaining branches in the decoder unless they help in increasing speed by resulting in a smaller executable.

\section{Architecture-specific Optimizations}

First, the decoder was updated to take advantage of AVX2 instructions when available. These new instructions benefit the fixed-point implementation as they allow simultaneous operations on 32 8-bit integers.

Second, the implementation of some nodes were hand-optimized to better take advantage of the processor architecture. For example, the SPC node was mostly rewritten. Listing 2 shows a small but critical subsection of the SPC node calculations where the index within a SIMD vector corresponding to the specified value is returned. The reduction operation required by the Repetition node has also been optimized manually.

Third, for the floating-point implementation, $\beta$ was changed to be in $\{+0,-0\}$ instead of $\{+1,-1\}$. In the floating-point representation [52], the most significant bit only carries the in- 

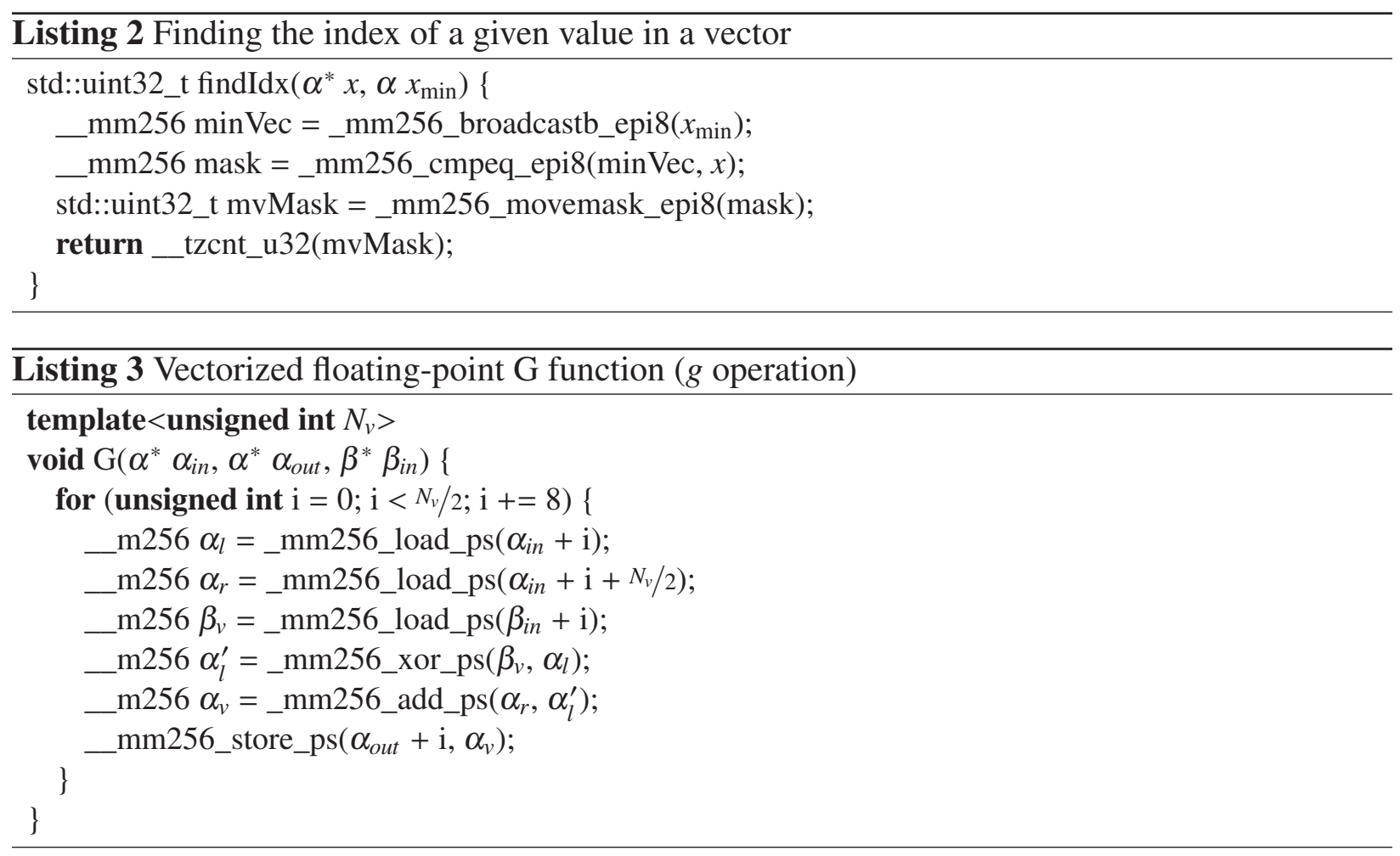

formation about the sign. Flipping this bit effectively changes the sign of the number. By changing the mapping for $\beta$, multiplications are replaced by faster bitwise XOR operations. Similarly, for the 8-bit fixed-point implementation, $\beta$ was changed to be in $\{0,-128\}$ to reduce the complexity of the Info and $\mathrm{G}$ functions.

Listings 3 and 4 show the resulting $\mathrm{G}$ functions for both the floating-point and fixed-point implementations as examples illustrating bottom-up optimizations used in our decoders.

\section{Memory Footprint}

The memory footprint is considered an important constraint for software applications. Our proposed implementations use 2 contiguous memory blocks that correspond to the $\alpha$ and $\beta$ values, respectively. The size of the $\beta$-memory is

$$
M_{\beta}=N W_{\beta}
$$

where $N$ is the frame length, $W_{\beta}$ is the number of bits used to store a $\beta$ value and $M_{\beta}$ is in bits. 


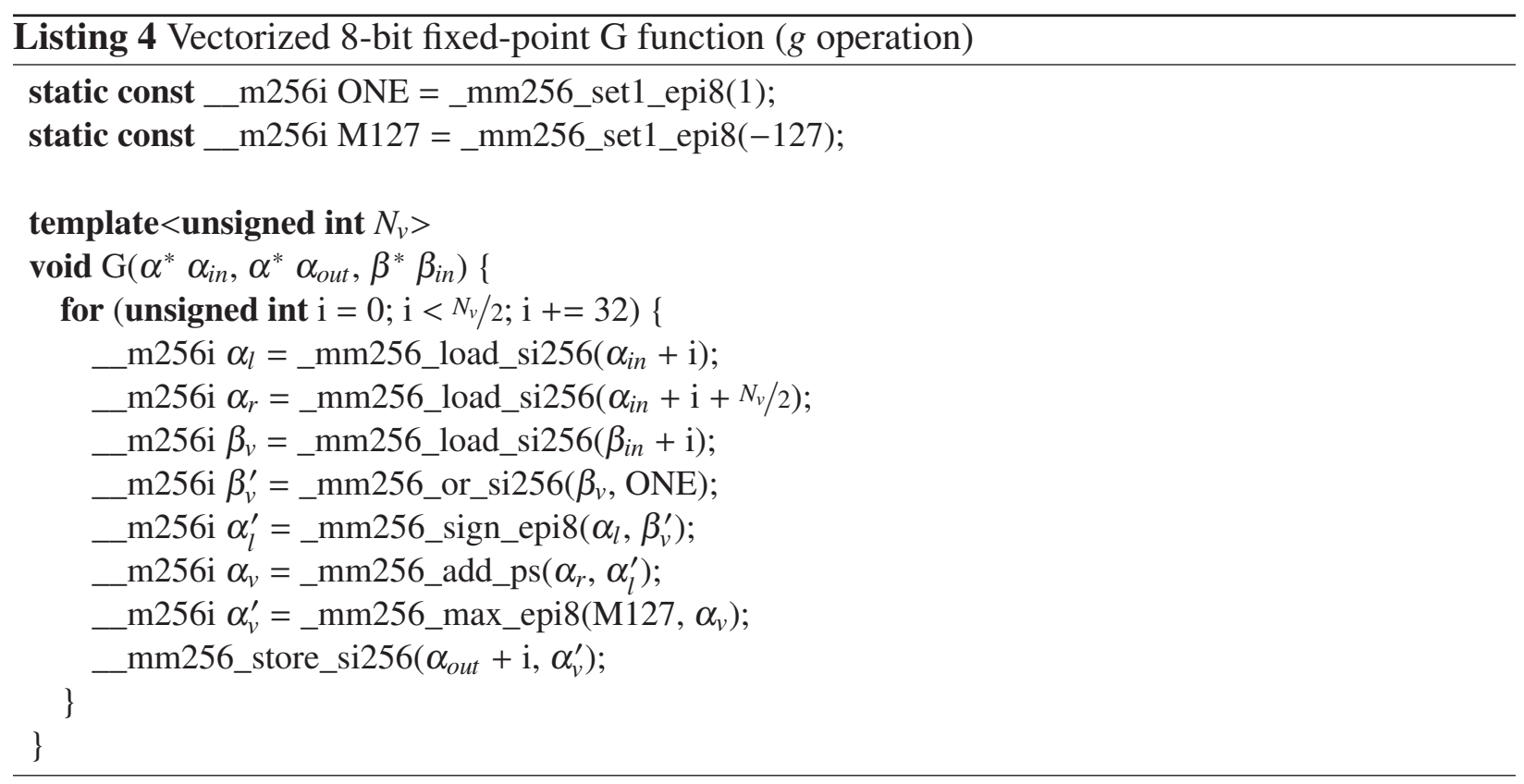

The size of the $\alpha$-memory can be expressed as

$$
M_{\alpha}=\left[(2 N-1)+A \log _{2} A-\left(\sum_{i=0}^{\log _{2}(A)-1} 2^{i}\right)\right] W_{\alpha}
$$

where $N$ is the frame length, $W_{\alpha}$ is the number of bits used to store an $\alpha$ value, $A$ is the number of $\alpha$ values per SIMD vector and $M_{\alpha}$ is in bits. Note that the expression of $M_{\alpha}$ contains the expression for the overhead $M_{\alpha \mathrm{OH}}$ due to tightly packing the $\alpha$ values as described in Section 4.2.2:

$$
M_{\alpha \mathrm{OH}}=\left[A \log _{2} A-\left(\sum_{i=0}^{\log _{2}(A)-1} 2^{i}\right)\right] W_{\alpha} .
$$

The memory footprint can thus be expressed as

$$
\begin{aligned}
M_{\text {total }} & =M_{\beta}+M_{\alpha} \\
& =N W_{\beta}+\left[(2 N-1)+A \log _{2} A-\left(\sum_{i=0}^{\log _{2}(A)-1} 2^{i}\right)\right] W_{\alpha} .
\end{aligned}
$$


Table 4.2: Decoding polar codes with floating-point precision using SIMD, comparing the instruction-based decoder (ID) with the unrolled decoder (UD).

\begin{tabular}{cccccc}
\hline \multirow{2}{*}{$\begin{array}{c}\text { Code } \\
(N, k)\end{array}$} & \multicolumn{2}{c}{ Info T/P $(\mathrm{Mbps})$} & & \multicolumn{2}{c}{ Latency $(\mu \mathrm{s})$} \\
\cline { 5 - 6 } & ID & UD & & ID & UD \\
\hline$(2048,1024)$ & 75.6 & 229.8 & & 14 & 4 \\
$(2048,1707)$ & 173.9 & 492.2 & & 10 & 3 \\
$(32768,27568)$ & 124.3 & 271.3 & & 222 & 102 \\
$(32768,29492)$ & 160.1 & 315.1 & & 184 & 94 \\
\hline
\end{tabular}

The memory footprint in kilobytes can be approximated with

$$
M_{\text {total (kbytes) }} \approx \frac{N\left(W_{\beta}+2 W_{\alpha}\right)}{8000} .
$$

\section{Implementation Comparison}

We first compare the SIMD-float results for this implementation-the unrolled decoder-with those from Section 4.2.1-the instruction-based decoder. Then we show SIMD-int8 results and compare them with that of the software decoder of Le Gal et. al [49]. As in the previous sections, the results are for an Intel Core i7-4770S running at $3.1 \mathrm{GHz}$ when Turbo is disabled and at up to 3.9 GHz otherwise. The decoders were limited to a single CPU core.

Table 4.2 shows the impact of the optimizations introduced in the unrolled version on the SIMD-float implementations. It resulted in the unrolled decoders being 2 to 3 times faster than the flexible, instruction-based, ones. Comparing Tables 4.1 and 4.2 shows an improvement factor from 3.3 to 5.7 for the SIMD-int8 implementations. It should be noted that some of the improvements introduced in the unrolled decoders could be backported to the instruction-based decoders, and is considered for future work.

Compared to the software polar decoders of [49], Table 4.3 shows that our throughput is lower for short frames but can be comparable for long frames. However, latency is an order of magnitude lower for all code lengths. This is to be expected as the decoders of [49] do inter-frame parallelism i.e. parallelize the decoding of independent frames while we parallelize the decoding of a frame. The memory footprint of our decoder is shown to be approximately 24 times lower than that of [49]. The results in [49] were presented with Turbo frequency boost enabled; therefore we present two sets of results for our proposed decoder: one with Turbo enabled, indicated by the asterisk (*) 
Table 4.3: Comparison of the proposed software decoder with that of [49].

\begin{tabular}{|c|c|c|c|c|c|c|c|}
\hline Decoder & Target & $\begin{array}{c}\text { L3 } \\
\text { Cache }\end{array}$ & $\begin{array}{c}f \\
(\mathrm{GHz})\end{array}$ & $\begin{array}{l}\text { Code } \\
(N, k)\end{array}$ & $\begin{array}{l}\text { Mem. footprint } \\
\quad \text { (kbytes) }\end{array}$ & $\begin{array}{c}\text { Info T/P } \\
\text { (Mbps) }\end{array}$ & $\begin{array}{l}\text { Latency } \\
\qquad(\mu \mathrm{s})\end{array}$ \\
\hline \multirow[t]{4}{*}[49]{$^{*}$} & Intel Core i7-4960HQ & $6 \mathrm{MB}$ & $3.6+$ & $(2048,1024)$ & 144 & 1,320 & 25 \\
\hline & & & & $(2048,1707)$ & 144 & 2,172 & 26 \\
\hline & & & & $(32768,27568)$ & 2304 & 1,232 & 714 \\
\hline & & & & $(32768,29492)$ & 2304 & 1,557 & 605 \\
\hline \multirow[t]{4}{*}{ this work } & Intel Core i7-4770S & $8 \mathrm{MB}$ & 3.1 & $(2048,1024)$ & 6 & 398 & 3 \\
\hline & & & & $(2048,1707)$ & 6 & 1,041 & 2 \\
\hline & & & & $(32768,27568)$ & 98 & 886 & 31 \\
\hline & & & & $(32768,29492)$ & 98 & 1,131 & 26 \\
\hline \multirow[t]{4}{*}{ this work* } & Intel Core i7-4770S & $8 \mathrm{MB}$ & $3.1+$ & $(2048,1024)$ & 6 & 502 & 2 \\
\hline & & & & $(2048,1707)$ & 6 & 1,293 & 1 \\
\hline & & & & $(32768,27568)$ & 98 & 1,104 & 25 \\
\hline & & & & $(32768,29492)$ & 98 & 1,412 & 21 \\
\hline
\end{tabular}

*Results with Turbo enabled.

and the 3.1+ GHz frequency in the table, and one with Turbo disabled. The results with Turbo disabled are more indicative of a full SDR system as all CPU cores will be fully utilized, not leaving any thermal headroom to increase the frequency. The maximum Turbo frequencies are 3.8 $\mathrm{GHz}$ and 3.9 GHz for the i7-4960HQ and i7-4770S CPUs, respectively.

Looking at the first two, or last two rows of Table 4.2, it can be seen that for a fixed code length, the decoding latency is smaller for higher code rates. The tendency of decoding latency to decrease with increasing code rate and length was first discussed in [27]. It was noted that higher rate codes resulted in SSC decoder trees with fewer nodes and, therefore, lower latency. Increasing the code length was observed to have a similar, but lesser, effect. However, once the code becomes sufficiently long, the limited memory bandwidth and number of processing resources form bottlenecks that negate the speed gains.

The effects of unrolling and using the Fast-SSC algorithm instead of SC are illustrated in Table 4.4. It can be observed that unrolling the Fast-SSC decoder results in a 5 time decrease in latency. Using the Fast-SSC instead of SC decoding algorithm decreased the latency of the unrolled decoder by 3 times. 
Table 4.4: Effect of unrolling and algorithm choice on decoding speed of the $(2048,1707)$ code on the Intel Core i7-4770S

\begin{tabular}{ccc}
\hline Decoder & Info T/P $(\mathrm{Mbps})$ & Latency $(\mu \mathrm{s})$ \\
\hline ID Fast-SSC & 210 & 8.1 \\
UD SC & 363 & 4.7 \\
UD Fast-SSC & 1041 & 1.6 \\
\hline
\end{tabular}

\subsection{Implementation on Embedded Processors}

Many of the current embedded processors used in SDR applications also offer SIMD extensions, e.g. NEON for ARM processors. All the strategies used to develop an efficient x86 implementation can be applied to the ARM architecture with changes to accommodate differences in extensions. For example, on ARM, there is no equivalent to the movemask SSE/AVX x86 instruction.

The equations for the memory footprint provided in Section 4.2.2 also apply to our decoder implementation for embedded processors.

\section{Comparison with Similar Works}

Results were obtained using the ODROID-U3 board, which features a Samsung Exynos 4412 System on Chip (SoC) implementing an ARM Cortex A9 clocked at $1.7 \mathrm{GHz}$. Like in the previous sections, the decoders were only allowed to use one core. Table 4.5 shows the results for the proposed unrolled decoders and provides a comparison with [48]. As with their desktop CPU implementation of [49], inter-frame parallelism is used in the latter.

It can be seen that the proposed implementations provide better latency and greater throughput at native frequencies. Since the ARM CPU in the Samsung Exynos 4412 is clocked at $1.7 \mathrm{GHz}$ while that in the NVIDIA Tegra 3 used in [48] is clocked at $1.4 \mathrm{GHz}$, we also provide linearly scaled throughput and latency numbers for the latter work, indicated by an asterisk (*) in the table. Compared to the scaled results of [48], the proposed decoder has 1.4-2.25 times the throughput and its latency is $25-36$ times lower. The memory footprint of our proposed decoder is approximately 12 times lower than that of [48]. Both implementations are using 8-bit fixed-point values. 
Table 4.5: Decoding polar codes with 8-bit fixed-point numbers on an ARM Cortex A9 using NEON.

\begin{tabular}{|c|c|c|c|c|c|}
\hline \multirow{2}{*}{$\begin{array}{l}\text { Code } \\
(N, k)\end{array}$} & \multirow{2}{*}{ Decoder } & \multirow{2}{*}{$\begin{array}{l}\text { Mem. } \\
\text { Footprint } \\
\text { (kBytes) }\end{array}$} & \multicolumn{2}{|c|}{$\mathrm{T} / \mathrm{P}(\mathrm{Mbps})$} & \multirow{2}{*}{$\begin{array}{l}\text { Latency } \\
\quad(\mu \mathrm{s})\end{array}$} \\
\hline & & & Coded & $\overline{\text { Info }}$ & \\
\hline \multirow[t]{3}{*}{$(1024,512)$} & [48] & 38 & 70.5 & 35.3 & 232 \\
\hline & {$[48]^{*}$} & 38 & 80.6 & 42.9 & 191 \\
\hline & this work & 3 & 113.1 & 56.6 & 9 \\
\hline \multirow[t]{3}{*}{$(32768,29492)$} & [48] & 1,216 & 33.1 & 29.8 & 15,844 \\
\hline & {$[48]^{*}$} & 1,216 & 40.2 & 36.2 & 13,048 \\
\hline & this work & 98 & 90.8 & 81.7 & 361 \\
\hline
\end{tabular}

*Results linearly scaled for the clock frequency difference.

\subsection{Implementation on Graphical Processing Units}

Most recent GPUs have the capability to do calculations that are not related to graphics. These GPUs are often called GPGPU. In this section, we describe our approach to implement software polar decoders in CUDA C [53] and present results for these decoders running on a NVIDIA Tesla K20c.

Most of the optimization strategies cited above could be applied or adapted to the GPU. However, there are noteworthy differences. Note that, when latency is mentioned below we refer to the decoding latency including the delay required to copy the data in and out of the GPU.

\subsubsection{Overview of the GPU Architecture and Terminology}

A NVIDIA GPU has multiple microprocessors with 32 cores each. Cores within the same microprocessor may communicate and share a local memory. However, synchronized communication between cores located in different microprocessors often has to go through the CPU and is thus costly and discouraged [54].

GPUs expose a different parallel programming model than general purpose processors. Instead of SIMD, the GPU model is single-instruction-multiple-threads (SIMT). Each core is capable of running a thread. A computational kernel performing a specific task is instantiated as a block. Each block is mapped to a microprocessor and is assigned one thread or more.

As it will be shown in Sect. 4.4.3, the latency induced by transferring data in and out of a GPU 


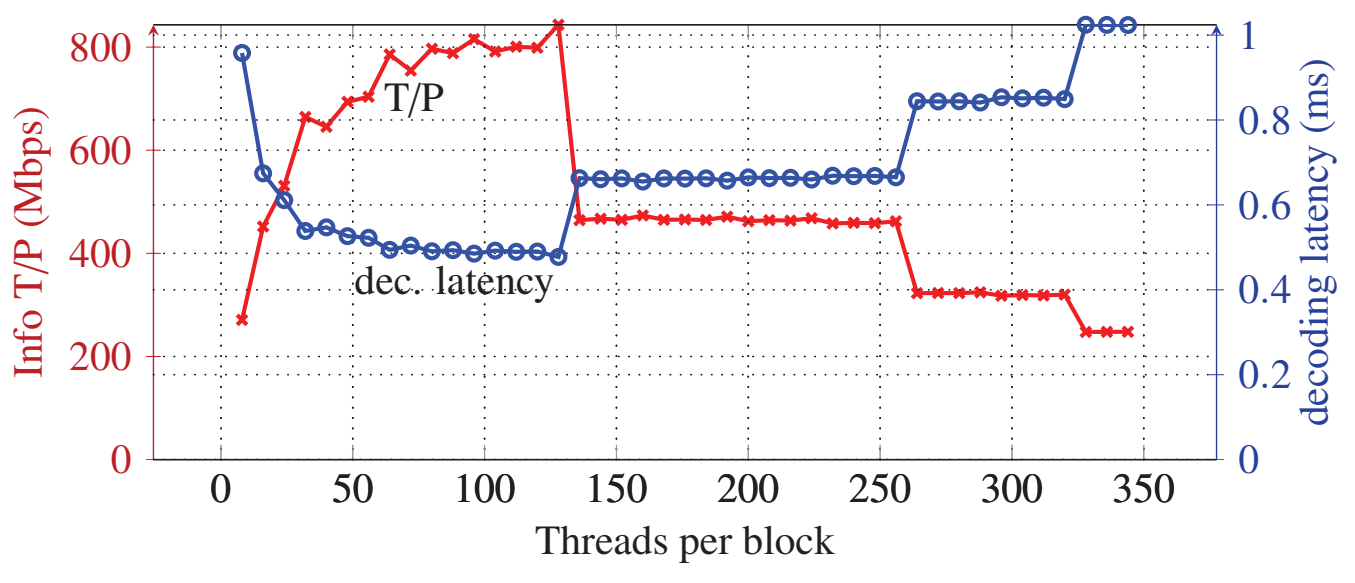

Figure 4.3: Effect of the number of threads per block on the information throughput and decoding latency for a $(1024,922)$ polar code where the number of blocks per kernel is 208.

is high. To minimize decoding latency and maximize throughput, a combination of intra- and interframe parallelism is used for the GPU contrary to the CPUs where only the former was applied. We implemented a kernel that decodes a single frame. Thus, a block corresponds to a frame and attributing e.g. 10 blocks to a kernel translates into the decoding of 10 frames in parallel.

\subsubsection{Choosing an Appropriate Number of Threads per Block}

As stated above, a block can only be executed on one microprocessor but can be assigned many threads. However, when more than 32 threads are assigned to a block, the threads starting at 33 are queued for execution. Queued threads are executed as soon as a core is free.

Fig. 4.3 shows that increasing the number of threads assigned to a block is beneficial only until a certain point is reached. For the particular case of a $(1024,922)$ code, associating more than 128 threads to a block negatively affects performance. This is not surprising as the average node width for that code is low at 52 .

\subsubsection{Choosing an Appropriate Number of Blocks per Kernel}

Memory transfers from the host to the GPU device are of high throughput but initiating them induces a great latency. The same is also true for transfers in the other direction, from the device to the host. Thus, the number of distinct transfers have to be minimized. The easiest way to do so is to run a kernel on multiple blocks. For our application, it translates to decoding multiple frames 


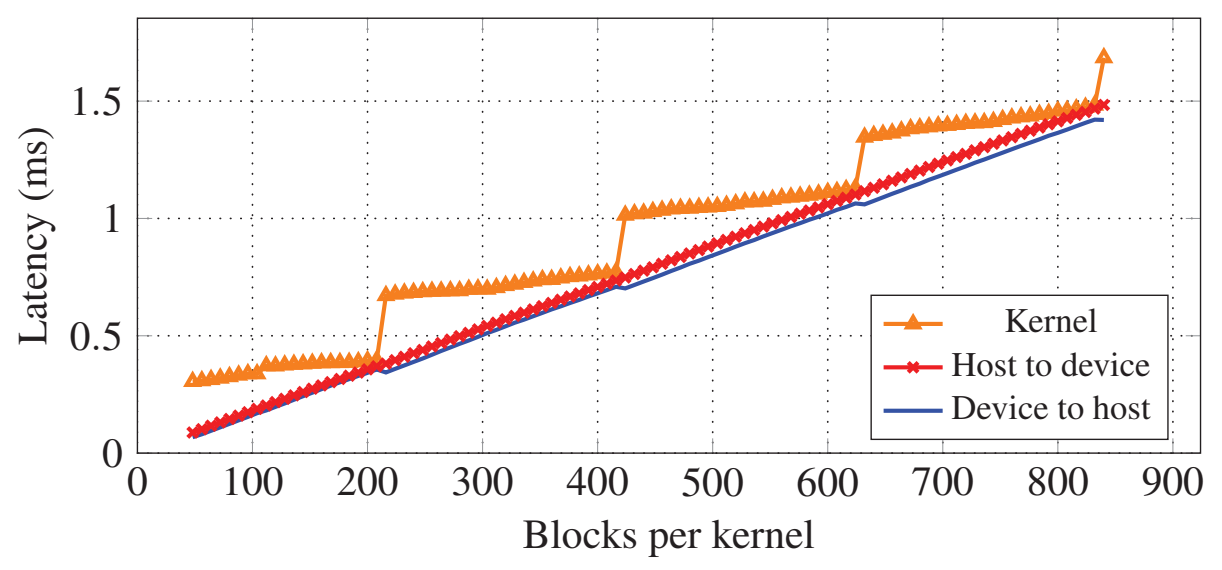

Figure 4.4: Effect of the number of blocks per kernel on the data transfer and kernel execution latencies for a $(2048,1707)$ polar code where the number of threads per block is 128 .

in parallel as a kernel decodes one frame.

Yet, there is a limit to the number of resources that can be used to execute a kernel i.e. decode a frame. At some point, there will not be enough computing resources to do the work in one pass and many passes will be required. The NVIDIA Tesla K20c card features the Kepler GK110 GPU that has 13 microprocessors with 32 cores and 16 load and store units each [55]. In total, 416 arithmetic or logic operations and 208 load or store operations can occur simultaneously.

Fig. 4.4 shows the latency to execute a kernel, to transfer memory from the host to the GPU and vice versa for a given number of blocks per kernel. The number of threads assigned per block is fixed to 128 and the decoder is built for a $(2048,1707)$ polar code. It can be seen that the latency of memory transfers grows linearly with the number of blocks per kernel. The kernel latency however has local minimums at multiples of 208. We conclude that the minimal decoding latency, the sum of all three latencies illustrated in Fig. 4.4, is bounded by the number of load and store units.

\subsubsection{On the Constituent Codes Implemented}

Not all the constituent codes supported by the general purpose processors are beneficial to a GPU implementation. In a SIMT model, reduction operations are costly. Moreover, if a conditional execution leads to unbalanced threads, performance suffers. Consequently, all nodes based on the SPC codes, that features both characteristics, are not used in the GPU implementation.

Experiments have shown that implementing the SPC node results in a throughput reduction by a factor of 2 or more. 


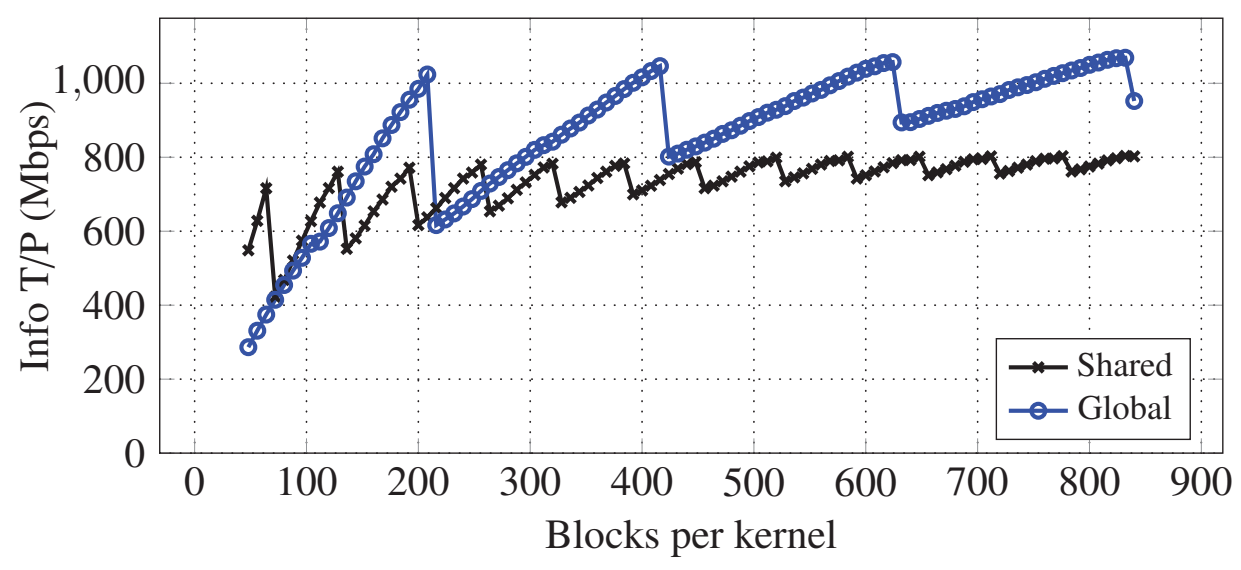

Figure 4.5: Information throughput comparison for a $(1024,922)$ polar code where intermediate results are stored in shared or global memory. The number of threads per block is 128 .

\subsubsection{Shared Memory and Memory Coalescing}

Each microprocessor contains shared memory that can be used by all threads in the same block. The NVIDIA Tesla K20c has $48 \mathrm{kB}$ of shared memory per block. Individual reads and writes to the shared memory are much faster than accessing the global memory. Thus, intuitively, when conducting the calculations within a kernel, it seems preferable to use the shared memory as much as possible in place of the global memory.

However, as shown by Fig. 4.5, it is not always the case. When the number of blocks per kernel is small, using the shared memory provides a significant speedup. In fact, with 64 blocks per kernel, using shared memory results in a decoder that has more than twice the throughput compared to a kernel that only uses the global memory. Past a certain value of blocks per kernel though, solely using the global memory is clearly advantageous for our application.

These results suggest that the GPU is able to efficiently schedule memory transfers when the number of blocks per kernel is sufficiently high.

\subsubsection{Asynchronous Memory Transfers and Multiple Streams}

Transferring memory from the host to the device and vice versa induces a latency that can be equal to the execution of a kernel. Fortunately, that latency can be first reduced by allocating pinned or page-locked host memory. As page-locked memory can be mapped into the address space of the device, the need for a staging memory is eliminated [53]. 
More significantly, NVIDIA GPUs with compute capability of 2.0 or above are able to transfer memory in and out of the device asynchronously. By creating three streams-sequences of operations that get executed in issue-order on the GPU-memory transfers and execution of the kernel can be overlapped, effectively multiplying throughput by a factor of 3 .

This also increases the memory footprint by a factor of three. On the GPU, the memory footprint is

$$
M_{\text {total (kbytes) }}=\frac{N\left(W_{\beta}+W_{\alpha}\right) B S}{8000},
$$

where $B$ is the number of blocks per kernel-i.e. the number of frames being decoded simultaneously-, $S$ is the number of streams, and where $W_{\beta}$ and $W_{\alpha}$ are the number of bits required to store a $\beta$ and an $\alpha$ value, respectively. For best performance, as detailed in the next section, both $\beta$ and $\alpha$ values are represented with floating-point values and thus $W_{\beta}=W_{\alpha}=32$.

\subsubsection{On the Use of Fixed-Point Numbers on a GPU}

It is tempting to move calculations to 8-bit fixed-point numbers in order to speedup performance, just like we did with the other processors. However, GPUs are not optimized for calculations with integers. Current GPUs only support 32-bit integers. Even so, the maximum number of operations per clock cycle per multiprocessor as documented by NVIDIA [53] clearly shows that integers are third class citizens behind single- and double-precision floating-point numbers. As an example, Table 2 of [53] shows that GPUs with compute capability 3.5-like the Tesla K20c-can execute twice as many double-precision floating-point multiplications in a given time than it can with 32bit integers. The same GPU can carry on 6 times more floating-point precision multiplications than its 32-bit integer counterpart.

\subsubsection{Results}

Table 4.6 shows the estimated information throughput and measured latency obtained by decoding various polar codes on a GPU. The throughput is estimated by assuming that the total memory transfer latencies are twice the latency of the decoding. This has been verified to be a reasonable assumption, using NVIDIA's profiler tool, when the number of blocks maximizes throughput.

Performing linear regression on the results of Table 4.6 indicates that the latency scales linearly with the number of blocks, leading to standard error values of $0.04,0.04$ and 0.14 for the $(1024,922),(2048,1707)$ and $(4096,3686)$ polar codes, respectively. In our decoder, a block cor- 
Table 4.6: Decoding polar codes on an NVIDIA Tesla K20c.

\begin{tabular}{cccc}
\hline$(N, k)$ & Nbr of blocks & Info T/P (Mbps) & Latency $(\mathrm{ms})$ \\
\hline$(1024,922)$ & 208 & 1,022 & 0.6 \\
& 416 & 1,046 & 1.1 \\
& 624 & 1,060 & 1.6 \\
$(2048,1707)$ & 832 & 1,070 & 2.2 \\
& 208 & 915 & 1.1 \\
& 416 & 936 & 2.2 \\
$(4096,3686)$ & 624 & 953 & 3.3 \\
& 832 & 964 & 4.5 \\
& 416 & 959 & 2.6 \\
& 624 & 1,002 & 4.9 \\
& 832 & 1,026 & 6.9 \\
\hline
\end{tabular}

responds to the decoding a single frame. The frames are independent of each other, and so are blocks. Thus, our decoder scales well with the number of available cores.

Furthermore, looking at Table 4.6 it can be seen that the information throughput is in the vicinity of a gigabit per second. Experiments have shown that the execution of two kernels can slightly overlap, making our throughput results of Table 4.6 worst-case estimations. For example, while the information throughput to decode 832 frames of a $(4096,3686)$ polar code is estimated at 1,043 Mbps in Table 4.6, the measured average value in NVIDIA's profiler tool was 1,228 Mbps, a $18 \%$ improvement over the estimated throughput.

Our experiments have also shown that our decoders are bound by the data transfer speed that this test system is capable of. The PCIe 2.0 standard [56] specifies a peak data throughput of 64 Gbps when 16 lanes are used and once 8b10b encoding is accounted for. Decoding 832 frames of a polar code of length $N=4096$ requires the transfer of 3,407,872 LLRs expressed as 32-bit floating-point numbers for a total of approximately 109 Mbits. Without doing any computation on the GPU, our benchmarks measured an average PCIe throughput of 45 Gbps to transfer blocks of data of that size from the host to the device and back. Running multiple streams and performing calculations on the GPU caused the PCIe throughput to drop to $40 \mathrm{Gbps}$. This corresponds to 1.25 Gbps when 32-bit floats are used to represent LLR inputs and estimated-bit outputs of the decoder. In light of these results, we conjecture that the coded throughput will remain approximately the 
Table 4.7: Comparison of the power consumption and energy per information bit for the (2048, 1707) polar code.

\begin{tabular}{ccccccc}
\hline Decoder & Target & $\begin{array}{c}\text { Mem. Footprint } \\
\text { (kbytes) }\end{array}$ & $\begin{array}{c}\text { Info. T/P } \\
(\text { Gbps })\end{array}$ & $\begin{array}{c}\text { Latency } \\
(\mu \mathrm{s})\end{array}$ & $\begin{array}{c}\text { Power } \\
(\mathrm{W})\end{array}$ & $\begin{array}{c}\text { Energy } \\
(\mathrm{nJ} / \text { info. bit) }\end{array}$ \\
\hline \multirow{2}{*49]}{} & Intel Core i7-4960HQ* & 144 & 2.2 & 26 & 13 & 6 \\
this work & Intel Core i7-4770S & 6 & 1.0 & 2 & 3 & 3 \\
& Intel Core i7-4770S* & 6 & 1.3 & 1 & 5 & 4 \\
& ARM Cortex A9 & 6 & 0.1 & 14 & 0.8 & 7 \\
& NVIDIA Tesla K20c & $3,408^{\dagger}$ & 0.9 & 1100 & 108 & 118 \\
\hline
\end{tabular}

*Results with Turbo enabled.

${ }^{\dagger}$ Amount required per stream. Three streams are required to sustain this throughput.

same for any polar code as the PCIe link is saturated and data transfer is the bottleneck.

\subsection{Energy Consumption Comparison}

In this section the energy consumption is compared for all three processor types: the desktop processor, the embedded processor and the GPU. Unfortunately the Samsung Exynos 4412 SoC does not feature sensors allowing for power usage measurements of the ARM processor cores. The energy consumption of the ARM processor was estimated from board-level measurements. An Agilent E3631A DC power supply was used to provide the 5V input to the ODROID-U3 board and the current as reported by the power supply was used to calculated the power usage when the processor was idle and under load.

On recent Intel processors, power usage can be calculated by accessing the Running Average Power Limit (RAPL) counters. The LIKWID tool suite [57] is used to measure the power usage of the processor. Numbers are for the whole processor including the Dynamic Random-Access Memory (DRAM) package. Recent NVIDIA GPUs also feature on-chip sensors enabling power usage measurement. Steady state values are read in real-time using the NVIDIA Management Libray (NVML) [58].

Table 4.7 compares the energy per information bit required to decode the $(2048,1707)$ polar code. The SIMD-int8 implementation of our unrolled decoder is compared with that of the implementation in [49]. The former uses an Intel Core i7-4770S clocked at $3.1 \mathrm{GHz}$. The latter uses an Intel Core i7-4960HQ clocked at $3.6 \mathrm{GHz}$ with Turbo enabled. The results for the ARM Cortex 
A9 embedded processor and NVIDIA Tesla K20c GPU are also included for comparison. Note that the GPU represents LLRs with floating-point numbers.

The energy per information bit is calculated with

$$
\mathrm{E}(J / \text { info. bit })=\frac{\mathrm{P}(W)}{\text { info. } \mathrm{T} / \mathrm{P}(\text { bits } / s)} .
$$

It can be seen that the proposed decoder is slightly more energy efficient on a desktop processor compared to that of [49]. For that polar code, the latter offers twice the throughput but at the cost of a latency that is at least 13 times greater. However, the latter is twice as fast for that polar code. Decoding on the embedded processor offers very similar energy efficiency compared to the Intel processor although the data throughput is an order of magnitude slower. However, decoding on a GPU is significantly less energy efficient than any of the decoders running on a desktop processor.

The power consumption on the embedded platform was measured to be fairly stable with only a $0.1 \mathrm{~W}$ difference between the decoding of polar codes of lengths 1024 or 32,768.

\subsection{Further Discussion}

\subsubsection{On the relevance of the instruction-based decoders}

Some applications require excellent error-correction performance that necessitates the use of polar codes much longer than $N=32,768$. For example, Quantum Key Distribution benefits from frames of $2^{21}$ to $2^{24}$ bits [59]. At such lengths, current compilers fail to compile an unrolled decoder. However, the instruction-based decoders are very suitable and are capable of throughput greater than 100 Mbps with a code of length 1 million.

\subsubsection{On the relevance of software decoders in comparison to hardware decoders}

The software decoders we have presented are good for systems that require moderate throughput without incurring the cost of dedicated hardware solutions. For example, in a SDR communication chain based on USRP radios and the GNU Radio software framework, a forward error-correction (FEC) solution using our proposed decoders only consumes 5\% of the total execution time on the receiver. Thus, freeing FPGA resources to implement functions other than FEC, e.g. synchronization and demodulation. 
By building such a setup to demonstrate one of our software polar encoder and decoder pair, we were awarded the first place of the microsystems experimental demonstration competition at the 2015 edition of the Innovation Day event jointly organized by the IEEE Circuits and Systems Society and the Regroupement Stratégique en Microélectronique du Québec.

\subsubsection{Comparison with LDPC codes}

LDPC codes are in widespread use in wireless communication systems. In this section, the errorcorrection performance of moderate-length polar codes is compared against that of standard LDPC codes [60]. Similarly, the performance of the state-of-the-art software LDPC decoders is compared against that of our proposed unrolled decoders for polar codes.

The fastest software LDPC decoders in literature are those of [14], which implements decoders for the 802.11 standard and present results for the Intel Core i7-2600 x86 processor. That wireless communication standard defines three code lengths: 1944, 1296, 648; and four code rates: 1/2, $2 / 3,3 / 4,5 / 6$. In [14], LDPC decoders are implemented for all four codes rates with a code length of 1944. A layered offset-min-sum decoding algorithm with five iterations is used and earlytermination is not supported.

Fig. 4.6 shows the FER of these codes using 10 iterations of a flooding-schedule offset minsum floating-point decoding algorithm which yields slightly better results than the five iteration layered algorithm used in [14]. The FER of polar codes with a slightly longer length of 2048 and matching code rates are also shown in Fig. 4.6.

Table 4.8 that provides the latency and information throughput for decoding 524,280 information bits using the state-of-the-art software LDPC decoders of [14] compared to our proposed polar decoders. To remain consistent with the result presented in [14], which used the Intel Core i7-2600 processor, the results in Table 4.8 use that processor as well.

While the polar code with rate $1 / 2$ offers a better coding gain than its LDPC counterpart, all other polar codes in Fig. 4.6 are shown to suffer a coding loss close to $0.25 \mathrm{~dB}$ at a FER of $10^{-3}$. However, as Table 4.8 shows, there is approximately an order of magnitude advantage for the proposed unrolled polar decoders in terms of both latency and throughput compared to the LDPC decoders of [14]. 


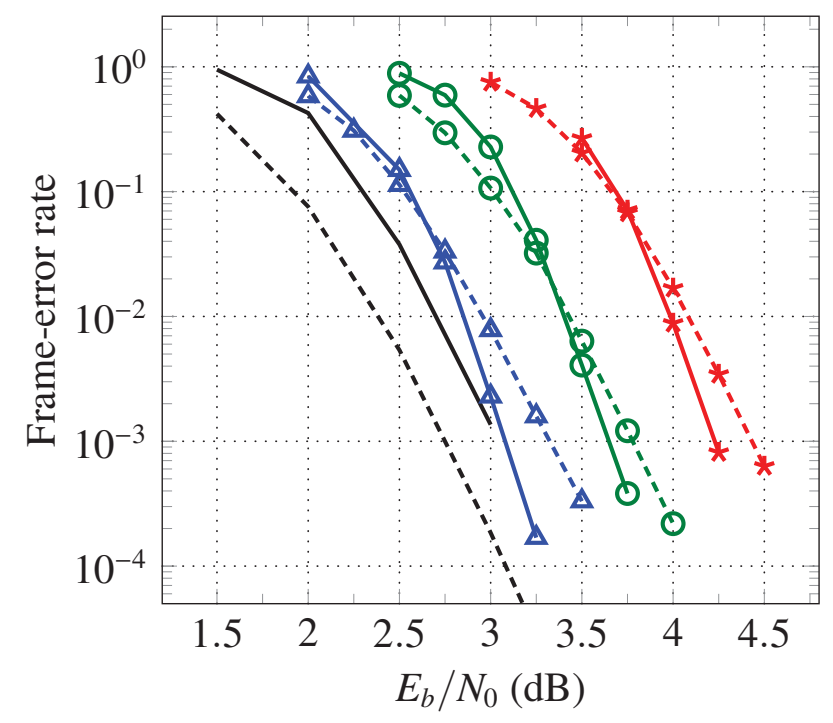

$\begin{array}{lllllll}\text { Polar: } \cdots & \mathrm{R}=1 / 2 & \cdots \mathrm{R}=2 / 3 & -\bullet & \mathrm{R}=3 / 4 & \cdots & \mathrm{R}=5 / 6 \\ \text { LDPC: } & -\mathrm{R}=1 / 2 & \rightarrow \mathrm{R}=2 / 3 & \rightarrow & \mathrm{R}=3 / 4 & \text { * } & \mathrm{R}=5 / 6\end{array}$

Figure 4.6: Error-correction performance of the polar codes of length 2048 compared with the LDPC codes of length 1944 from the 802.11n standard.

\subsection{Conclusion}

In this chapter, we presented low-latency software polar decoders adapted to different processor architectures. The decoding algorithm is adapted to exploit different SIMD instruction sets for the desktop and embedded processors (SSE, AVX and NEON) or to the SIMT model inherent to GPU processors. The optimization strategies go beyond parallelisation with SIMD or SIMT. Most notably, we proposed to generate a branchless fully unrolled decoder, to use compile-time specialization, and adopt a bottom-up approach by adapting the decoding algorithm and data representation to features offered by processor architectures. For desktop processors, we have shown that intra-frame parallelism can be exploited to get a very low-latency while achieving information throughputs greater than 1 Gbps using a single core. For embedded processors, the principle remains but the achievable information throughputs are more modest at $80 \mathrm{Mbps}$. On the GPU we showed that inter-frame parallelism could be successfully used in addition to intra-frame parallelism to reach better speed, and the impact of two critical parameters on the performance of the decoders was explored. We showed that given the right set of parameters, GPU decoders are able to sustain an information throughput around $1 \mathrm{Gbps}$ while simultaneously decoding hundreds 
Table 4.8: Information throughput and latency of the polar decoders compared with the LDPC decoders of [14] when estimating 524,280 information bits on a Intel Core i7-2600.

\begin{tabular}{cccccc}
\hline \multirow{2}{*}{ Decoder } & $N$ & Rate & \multicolumn{2}{c}{ Latency } & Info. T/P \\
\cline { 4 - 5 } & & & total $(\mathrm{ms})$ & per frame $(\mu \mathrm{s})$ & $(\mathrm{Mbps})$ \\
\hline \multirow{2}{*14]}{} & \multirow{2}{*}{1944} & $1 / 2$ & 17.4 & $\mathrm{~N} / \mathrm{A}$ & 30.1 \\
& & $2 / 3$ & 12.7 & $\mathrm{~N} / \mathrm{A}$ & 41.0 \\
& & $3 / 4$ & 11.2 & $\mathrm{~N} / \mathrm{A}$ & 46.6 \\
this work & \multirow{2}{*}{2048} & $5 / 6$ & 9.3 & $\mathrm{~N} / \mathrm{A}$ & 56.4 \\
& & $1 / 2$ & 2.0 & 3.83 & 267.4 \\
& & $2 / 3$ & 1.0 & 2.69 & 507.4 \\
& & $3 / 4$ & 0.8 & 2.48 & 619.4 \\
& $5 / 6$ & 0.6 & 2.03 & 840.9 \\
\hline
\end{tabular}

of frames. Finally, we showed that the memory footprint of our proposed decoder is at least an order of magnitude lower than that our the state-of-the-art polar decoder while being slightly more energy efficient. These results indicate that the proposed software decoders make polar codes interesting candidates for SDR applications. In fact, we won an award at a experimental demonstration competition by using our software solution in a over-the-air radio communication setup. 



\section{Chapter 5}

\section{Unrolled Hardware Architectures for Polar Decoders}

In this chapter, we demonstrate that polar decoders can achieve extremely high throughput values and retain moderate complexity. We present a family of architectures for hardware polar decoders using a reduced-complexity successive-cancellation decoding algorithm that employ unrolling. The resulting fully-unrolled architectures are capable of achieving a coded throughput in excess $400 \mathrm{Gbps}$ and 1 Tbps on an FPGA or an ASIC, respectively-two to three orders of magnitude greater than current state-of-the-art polar decoders-while maintaining a competitive energy efficiency of $6.9 \mathrm{pJ} / \mathrm{bit}$ on ASIC. Moreover, the proposed architectures are flexible in a way that makes it possible to explore the trade-off between area, throughput and energy efficiency. We present the associated results for a range of pipeline depths, and code lengths and rates. We also discuss how the throughput and complexity of decoders are effected when implemented for an I/O-bound system.

\subsection{Introduction}

Conventional polar decoders implement one or a few specialized computational units and reuse them multiple times during the decoding process [8], [35]-[38], [40]. It was shown in [50] that unrolling the decoding process can lead to significant speed improvements in software polar decoders.

The goal of this chapter is to show how unrolling and pipelining the decoder tree can lead to 
hardware architectures that can achieve throughput values greater than 1 Tbps on a $28 \mathrm{~nm}$ CMOS technology ASIC operating at $1 \mathrm{GHz}$ - three orders of magnitude faster than the state of the art. On FPGAs, the fastest architectures can reach hundreds of Gbps. Moreover, we present a family of architectures that offers a flexible trade-off between throughput, area and energy efficiency.

We start this chapter with Section 5.2 to provide a brief review of state-of-the-art polar decoder architectures. Section 5.3 presents the proposed family of architectures, and the operations and processing nodes used. It also specifies how code shortening can be used with the proposed architectures. Section 5.4 discusses the implementation and presents both FPGA and ASIC results for various code lengths and rates. Results are compared against state-of-the-art polar decoder implementations. The coded throughput of our decoders is shown to be in excess of $400 \mathrm{Gbps}$ for a $(2048,1024)$ polar code decoded on an FPGA and of 1 Tbps for a $(1024,512)$ polar code decoded on an ASIC, respectively two and three orders of magnitude over the current state of the art. Some power estimations are also provided for both FPGA and ASIC. Finally, Section 5.5 concludes this paper.

Preliminary results of this work were presented in a letter [20]. In this chapter, we generalize the architecture into a family of architectures offering a flexible trade-off between throughput, area and energy efficiency, give more details on the unrolled architecture and provide more results. We also significantly improve the $(1024,512)$ fully-unrolled deeply-pipelined polar decoder implementation results on all metrics. Finally, ASIC results for the $28 \mathrm{~nm}$ FD-SOI technology from STMicroelectronics are provided as well as power estimations for both FPGA and ASIC.

\subsection{State-of-the-Art Architectures with Implementations}

Most hardware polar decoder architectures presented in the literature, [8], [27], [35]-[38], [40], use the SC decoding algorithm or an SC-based algorithm. These decoders require little logic area (ASIC) or resource usage (FPGA). As an example, the fastest of these SC-based decoders, the Fast-SSC decoder of [8], utilizes a processor-like architecture where the different units are used one to many times over the decoding of a frame. With the algorithmic improvements reviewed in Section 2.6, the Fast-SSC decoder was shown to be capable of achieving a 1.2 Gbps coded throughput (1.1 Gbps information throughput) at $108 \mathrm{MHz}$ on an FPGA for a polar code with a length $N=2^{15}$.

Recently, two polar decoders capable of achieving a coded throughput greater than 1 Gbps with 
a short $(1024,512)$ polar code were proposed. An iterative BP fully-parallel decoder achieving a throughput of $4.7 \mathrm{Gbps}$ at $300 \mathrm{MHz}$ on a $65 \mathrm{~nm}$ CMOS ASIC was proposed in [15]. More recently, a fully-combinational, SC-based decoder with input and output registers was proposed in [47]. That decoder reaches a throughput of $2.9 \mathrm{Gbps}$ at $2.79 \mathrm{MHz}$ on a $90 \mathrm{~nm} \mathrm{CMOS} \mathrm{ASIC}$ and of 1.2 Gbps at $596 \mathrm{kHz}$ on a $40 \mathrm{~nm}$ CMOS Xilinx Virtex 6 FPGA.

While these results are a significant improvement, their throughput, less than 6 Gbps, is still under the projected minimal peak throughput for future 5G communication standards [61]-[63]. Therefore, in this paper we propose a family of architectures capable of achieving one to three orders of magnitude greater throughtput than the current state-of-the-art polar decoders.

\subsection{Architecture, Operations and Processing Nodes}

Similar to some decoders presented in the previous section, in order to significantly increase decoding throughput, our family of architectures does not focus on logic reuse but fully unrolls and pipelines the required calculations. A fully-unrolled decoder is a decoder where each and every operation or node required in estimating a codeword is instantiated with dedicated hardware. As an example, if a decoder for a specific polar code requires two executions of an $F$ operation with a length of 8 , a fully-unrolled decoder for that code will feature two $F$ modules with inputs of size 8 instead of reusing the same block twice.

The idea of fully unrolling a decoder has previously been applied to decoders for other families of error-correcting codes. Notably, in [64], [65], the authors propose a fully-unrolled deeplypipelined decoder for an LDPC code. Polar codes are more suitable to unrolling as they do not feature a complex interleaver like LDPC codes.

In this section, we provide details on the proposed family of architectures and describe the operations and processing nodes used by our architectures.

\subsubsection{Fully Unrolled (Basic Scheme)}

Building upon the work done on software polar decoders described in Chapter 4, we propose fully-unrolled hardware decoder architectures built for a specific polar code using a subset of the low-complexity Fast-SSC algorithm.

In the fully-unrolled architecture, all the nodes of a decoder tree exist simultaneously. Fig. 5.2 shows a fully-unrolled decoder for the $(8,4)$ polar code illustrated as a decoder tree in Fig. 5.1b. 


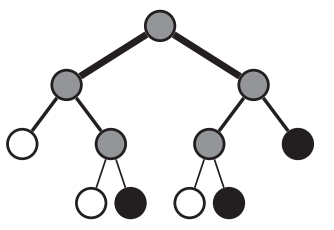

(a) SSC

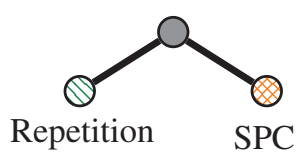

(b) Fast-SSC

Figure 5.1: Decoder trees for an $(8,4)$ polar code decoded with the (a) SSC and (b) Fast-SSC algorithms.

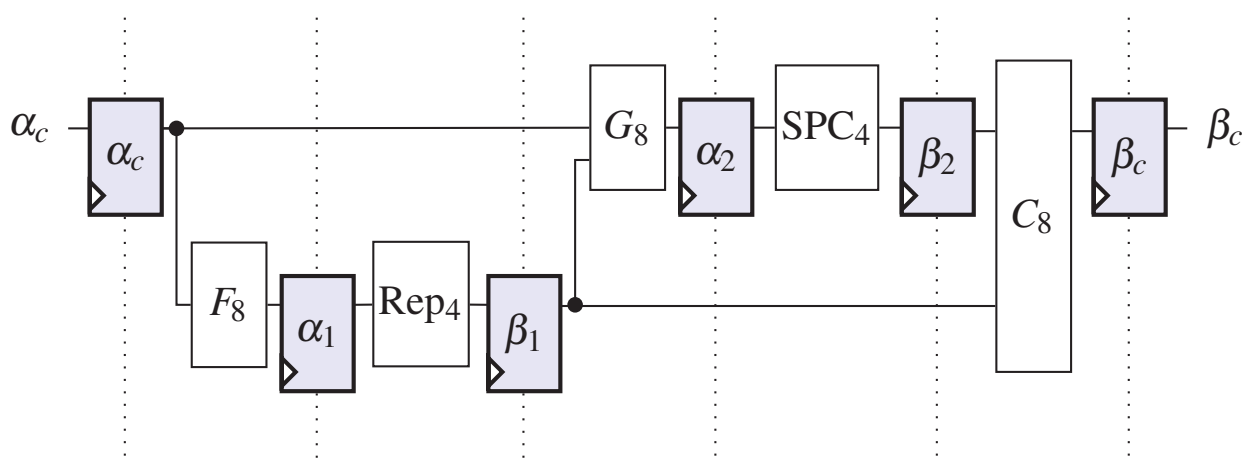

Figure 5.2: Fully-unrolled decoder for a $(8,4)$ polar code. Clock and enable signals omitted for clarity.

White blocks represent operations in the Fast-SSC algorithm and the subscripts of their labels correspond to their input length $N_{v}$. Rep denotes a Repetition node, and $C$ stands for the Combine operation. Grayed rectangles are registers. The clock and enable signals for those blocks are omitted for clarity. As it will be shown in Section 5.3.3, even with the multi-cycle paths, the enable signals for that decoder may always remain asserted without affecting the correctness as long as the input to the decoder remains stable for 3 clock cycles. This constitutes our basic scheme. On FPGA, it takes advantage of the fact that registers are available right after LUTs in logic blocks, meaning that adding a register after each operation does not require any additional logic block.

The code rate and frozen bit locations both affect the structure of the decoder tree and, in turn, the number of operations performed in a Fast-SSC decoder. However, as it will be shown in Section 5.4.4, the growth in logic usage or area for unrolled decoders remains $O(N \log N)$, where $N$ is the code length. 


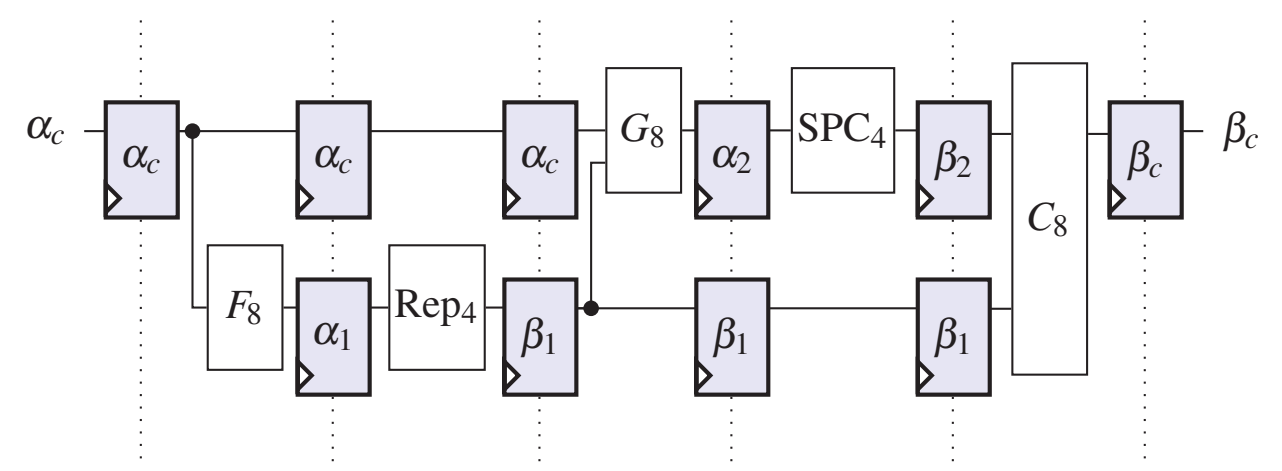

Figure 5.3: Fully-unrolled deeply-pipelined decoder for a $(8,4)$ polar code. Clock signals omitted for clarity.

\subsubsection{Deeply Pipelined}

In a deeply-pipelined architecture, a new frame is loaded into the decoder at every clock cycle. Therefore, a new estimated codeword is output at each clock cycle as each register is active at each rising edge of the clock (no enable signal required). In that architecture, at any point in time, there are as many frames being decoded as there are pipeline stages. This leads to a very high throughput at the cost of high memory requirements. Some pipeline stage paths do not contain any processing logic, only memory. They are added to ensure that the different messages remain synchronized. These added memories yield register chains, or SRAM blocks, as will be shown in Section 5.3.5.

The unrolled decoder of Fig. 5.2 can be transformed into a deeply-pipelined decoder by adding four registers. Two registers are needed to retain the channel LLRs, denoted $\alpha_{c}$ in the figure, during the $2^{\text {nd }}$ and $3^{\text {rd }}$ clock cycles. Similarly, two registers have to be added for the persistence of the hard-decision vector $\beta_{1}$ over the $4^{\text {th }}$ and $5^{\text {th }}$ clock cycles. Making these modifications results in the fully-unrolled deeply-pipelined decoder shown in Fig. 5.3. Fig. 5.4 shows another example of a fully-unrolled deeply-pipelined decoder, but for a $(16,14)$ polar code featuring more operations and node types compared to Fig. 5.3, where I denotes a Rate-1 node.

For this architecture, the amount of memory required is quadratic in code length and, similarly to resource usage, affected by rate and frozen bit locations. As will be shown in Section 5.4, this growth in memory usage limits the proposed deeply-pipelined architecture to codes of moderate lengths, under 4096 bits, at least for implementations using the target FPGA.

Information throughput is defined as $P f R$ bps, where $P$ is the width of the output bus in bits, $f$ is the execution frequency in $\mathrm{Hz}$ and $R$ is the code rate. In a deeply-pipelined architecture, $P$ 


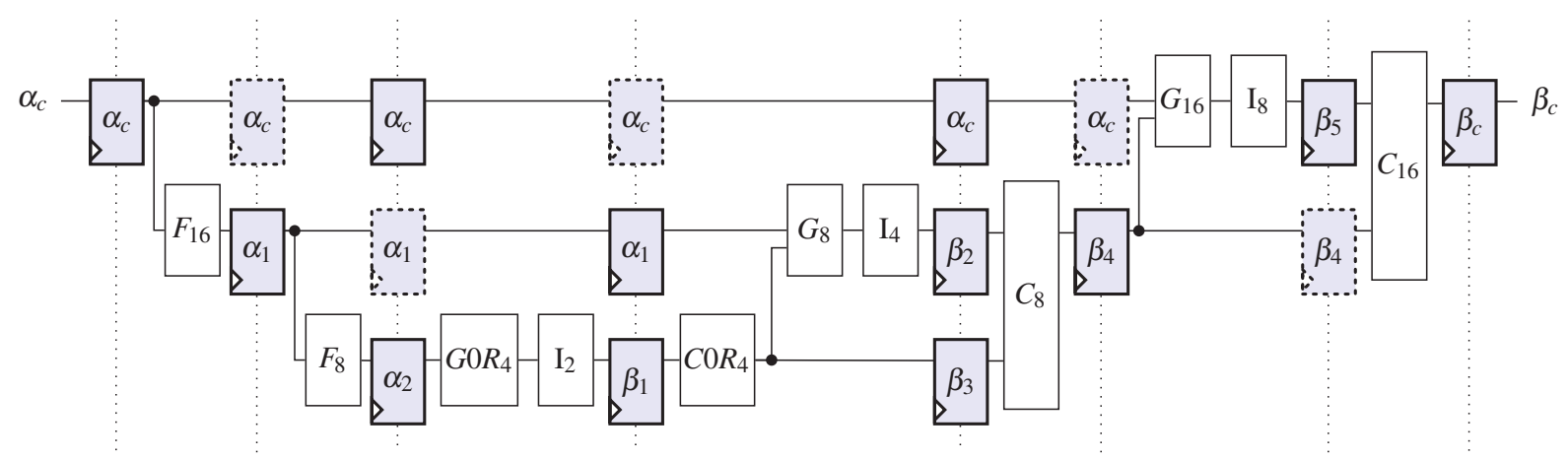

Figure 5.4: Fully-unrolled deeply-pipelined decoder for a $(16,14)$ polar code. Clock signals omitted for clarity.

is assumed to be equal to the code length $N$. The decoding latency depends on the frozen bit locations and the constrained maximum width for all processing nodes, but is less than $N \log _{2} N$. In our experiments, with the operations and optimizations described below, the decoding latency never exceeded $N / 2$ clock cycles.

\subsubsection{Partially Pipelined}

In a deeply-pipelined architecture, a significant amount of memory is required for data persistence. That memory quickly increases with the code length $N$. Instead of loading a new frame into the decoder and estimating a new codeword at every cycle, we propose a compromise where the unrolled decoder can be partially pipelined to reduce the required memory. Let $\mathcal{I}$ be the initiation interval, where a new estimated codeword is output every $I$ clock cycles. The case where $I=1$ translates to a deeply-pipelined architecture.

Setting $\mathcal{I}>1$ leads to a significant reduction in the memory requirements. An initiation interval of $\mathcal{I}$ translates to an effective required register chain length of $\lceil L / I\rceil$ instead of $L$, where $L$ is the length of the register chain. Using $I=2$ leads to a $\sim 50 \%$ reduction in the amount of memory required for that section of the circuit. This reduction applies to all register chains present in the decoder.

The unrolled decoder of Fig. 5.2 can be seen as a partially-pipelined decoder with an initiation interval $\mathcal{I}=3$. A partially-pipelined decoder with $\mathcal{I}=2$ can be obtained for a $(16,14)$ polar code by removing the dotted registers in Fig. 5.4, leading to the decoder shown in Fig. 5.5.

The initiation interval $\mathcal{I}$ can be increased further in order to reduce the memory requirements, 


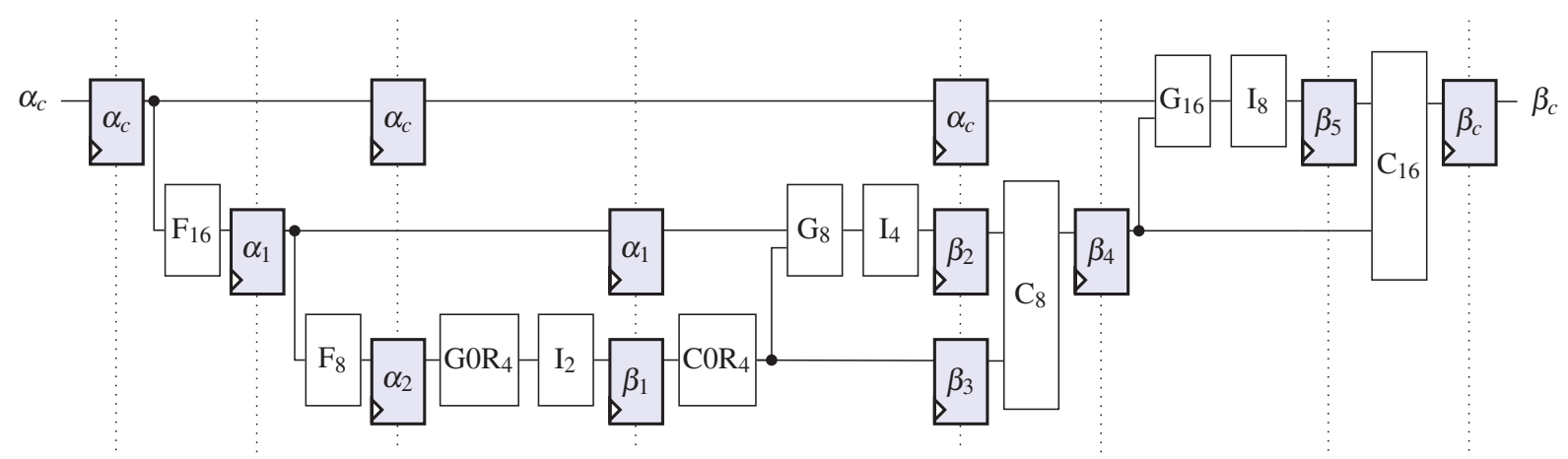

Figure 5.5: Fully-unrolled partially-pipelined decoder for a $(16,14)$ polar code with $\mathcal{I}=2$. Clock and enable signals omitted for clarity.

but only up to a certain limit (corresponding to the basic scheme). We call that limit the maximum initiation interval $\mathcal{I}_{\max }$, and its value depends on the decoder tree. By definition, the longest register chain in a fully-unrolled decoder is used to preserve the channel LLRs $\alpha_{c}$. Hence, the maximum initiation interval corresponds to the number of clock cycles required for the decoder to reach the last operation in the decoder tree that requires $\alpha_{c}, \mathrm{G}_{N}$, the operation calculated when going down the right edge linking the root node to its right-hand-side child. Once that $\mathrm{G}_{N}$ operation is completed, $\alpha_{c}$ is no longer needed and can be overwritten. As an example, consider the $(8,4)$ polar decoder illustrated in Fig. 5.2. As soon as the switch to the right-hand side of the decoder tree occurs, i.e. when $G_{8}$ is traversed, the register containing the channel LLRs $\alpha_{c}$ can be updated with the LLRs for the new frame without affecting the remaining operations for the current frame. Thus the maximum initiation interval, $I_{\max }$, for that decoder is 3 .

The resulting information throughput is $P f R / \mathcal{I}$ bps, where $\mathcal{I}$ is the initiation interval. Note that this new definition can also be used for the deeply-pipelined architecture. The decoding latency remains unchanged compared to the deeply-pipelined architecture.

The partially-pipelined architecture requires a more elaborate controller than the deeply-pipelined architecture. For both fully- and partially-pipelined architectures, the controller generates a done signal to indicate that a new estimated codeword is available at the output. For the partiallypipelined architecture, the controller also contains a counter with maximum value of $(I-1)$ which generates the $\mathcal{I}$ enable signals for the registers. An enable signal is asserted only when the counter reaches its value, in $[0, \mathcal{I}-1]$, otherwise it remains deasserted. Each register uses an enable signal corresponding to its location in the pipeline modulo $\mathcal{I}$. As an example, let us consider the decoder of Fig. 5.5, i.e. $\mathcal{I}$ is set to 2 . In that example, two enable signals are created and a simple 
counter alternates between 0 and 1 . The registers storing the channel LLRs $\alpha_{c}$ are enabled when the counter is equal to 0 because their input resides on the even ( 0,2 and 4$)$ stages of the pipeline. On the other hand, the two registers holding the $\alpha_{1}$ LLRs are enabled when the counter is equal to 1 because their inputs are on odd ( 1 and 3 ) stages. The other registers follow the same rule.

The required memory resources could be further reduced by performing the decoding operations in a combinational manner, i.e. by removing all the registers except the ones labeled $\alpha_{c}$ and $\beta_{c}$, as in [47]. However, the resulting reachable frequency is too low for the desired throughput level.

\subsubsection{Operations and Processing Nodes}

In order to keep the critical paths as short as possible, only a subset of the operations and processing nodes proposed in the original Fast-SSC algorithm are used. Furthermore, for some nodes, the maximum processing node length $N_{v}$ is constrained to smaller values than the ones used in [8].

Notably, the Repetition and SPC nodes are limited to $N_{v}=8$ and 4, respectively. The remainder of the operations-F, G, G0R, Combine, $C 0 R$ - are not constrained, as their lengths do not affect the critical paths. While it was not required in the original Fast-SSC algorithm, our architecture includes a Rate-1 processing node, implementing (2.4). That Rate-1 node is not constrained in length either.

In order to reduce latency and resource usage, improvements were made to some operations and nodes. They are detailed below.

COR Operations: A COR operation is a special case of a Combine operation (2.3) where the lefthand-side constituent code, $\beta_{l}$, is a Rate- 0 node. Thus, the operation is equivalent to copying the estimated hard values from the right-hand-side constituent code over to the left-hand side.

In other words, a $C O R$ does not require any logic, it only consists of wires. All occurrences of that operation were thus merged with the following modules, saving a clock cycle without negatively impacting the maximum clock frequency and reducing memory.

Rate-1 or Information Nodes: With a fixed-point number representation, a Rate-1 (or Information) node amounts to copying the most significant bit of the input LLRs. Similarly to the $C 0 R$ operation, the Information node does not require any logic and is equivalent to wires. 
Contrary to the $C 0 R$ operation though, we do not save a clock cycle by prepending the Information node to its consumer node. Instead, the register storing LLRs at the output of its producer is removed and the Information node is appended, along with its register used to store the hard decisions. Not only is the decoding latency reduced by a clock cycle, but a register storing LLR values is removed.

Repetition Nodes: The output of a Repetition node is a single bit estimate. The systematic polar decoder of [8] copies that estimated information bit $N_{v}$ times to form the estimated bit vector, before storing it. In our implementation, we store only one bit that is later expanded just before a consumer requires it. This reduces the width of register chains carrying bit estimates generated by Repetition nodes, thus decreasing resource usage.

\subsubsection{Replacing Register Chains with SRAM Blocks}

As the code length $N$ grows, long register chains start to appear in the decoder, especially with a smaller $\mathcal{I}$. In order to reduce the number of registers required, register chains can be converted into SRAM blocks.

Consider the register chain of length 6 used for the persistence of the channel LLRs $\alpha_{c}$ in the fully-unrolled deeply-pipelined $(16,14)$ decoder shown in top row of Fig. 5.4. That register chain can be replaced by an SRAM block with a depth of 6 along with a controller to generate the appropriate read and write addresses. Similar to a circular buffer, if the addresses are generated to increase every clock cycle, the write address is set to be one position ahead of the read address.

SRAM blocks can replace register chains in a partially-pipelined architecture as well. In both architectures, the SRAM block depth has to be equal or greater than the register chain length. The same constraint applies to the width.

In scenarios where narrow SRAM blocks are not desirable, register chains can be merged to obtain a wider SRAM block even if the register chains do not have the same length. If the lengths of 2 register chains to be merged differ, the first registers in the longest chain are preserved, and only the remaining registers are merged with the other chain. 


\subsection{Implementation and Results}

\subsubsection{Methodology}

In our experiments, decoders are built with sufficient memory to accommodate storing an extra frame at the input, and to preserve an estimated codeword at the output. As a result, the next frame can be loaded while a frame is being decoded. Similarly, an estimated codeword can be read while the next frame is being decoded. To facilitate comparison between the fully and partially-pipelined architectures, we define decoding latency to only include the time required for the decoder to decode a frame; loading channel LLRs and offloading estimated codewords are excluded from the calculations.

The quantization used was determined by running fixed-point simulations with bit-true models of the decoders. A smaller number of bits is used to store the channel LLRs compared to that of the other LLRs used in the decoder. All LLRs share the same number of fractional bits. We denote quantization as $Q_{i} \cdot Q_{c} \cdot Q_{f}$, where $Q_{c}$ is the total number of bits to store a channel LLR, $Q_{i}$ is total the number of bits used to store internal LLRs and $Q_{f}$ is the number of fractional bits in both. Fig. 5.6 shows the effect of quantization on the error-correction performance of a $(1024,512)$ polar code modulated with BPSK and transmitted over an AWGN channel. Note that more fixed-point combinations are illustrated compared to previous chapters. Looking at Fig. 5.6, it can be seen that using $Q_{i} \cdot Q_{c} \cdot Q_{f}$ equal to 5.4 .0 results in a $0.1 \mathrm{~dB}$ performance degradation at a BER of $10^{-6}$. Thus we used that quantization for the hardware results.

FPGA results are for an Altera Stratix IV EP4SGX530KH40C2 to facilitate comparison against most polar decoder implementations in the literature. That FPGA features 637,440 LUTs, 424,960 registers and 21,233,664 bits of SRAM. Better results are to be expected if more recent FPGAs were to be targeted. ASIC synthesis results for the $28 \mathrm{~nm}$ FD-SOI CMOS technology from STMicroelectronics are obtained by running Synopsys Design Compiler in topographical mode for a typical library at $1.0 \mathrm{~V}$ and $125^{\circ} \mathrm{C}$, and a timing constraint set to $1 \mathrm{~ns}$. Furthermore, on ASIC, only registers are used as we did not have access to an SRAM compiler.

\subsubsection{Effect of the Initiation Interval}

In this section, we explore the effect of the initiation interval on the implementation of the fullyunrolled architecture. The decoders are built for the same $(1024,512)$ polar code used in [20], although many improvements were made since the publication of that work (see Section 5.3.4). 

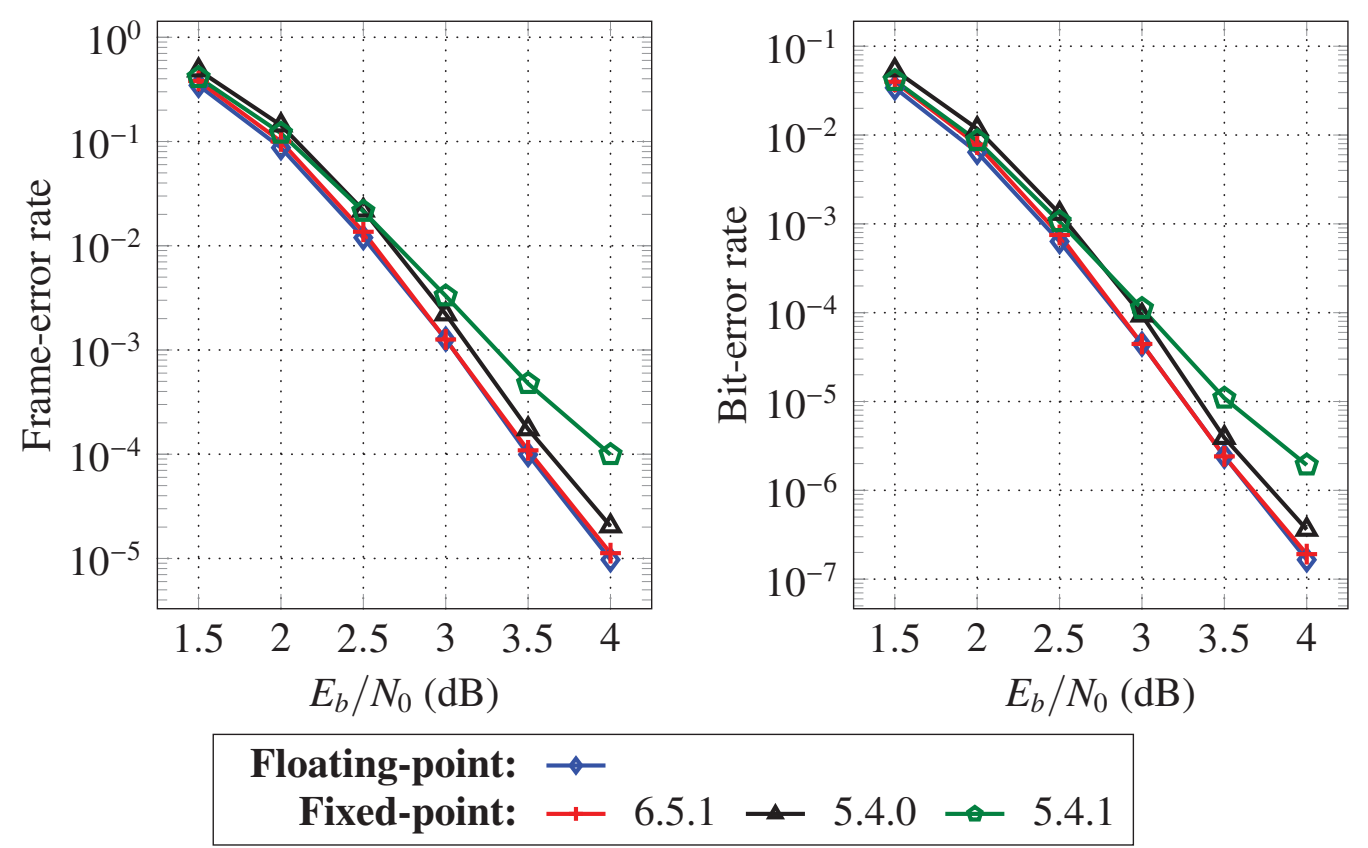

Figure 5.6: Effect of quantization on the error-correction performance of a $(1024,512)$ polar code.

Regardless of the initiation interval, all decoders use 5.4.0 quantization and have a decoding latency of 364 clock cycles.

Tables 5.1 and 5.2 show the results for various initiation intervals on the FPGA and ASIC implementations, respectively. Besides the effect on coded throughput, increasing the initiation interval causes a significant reduction in the FPGA resources required or of the ASIC area. On FPGA, while the throughput is approximately cut in half, using $\mathcal{I}=2$ reduces the number of required LUTs, registers and RAM bits by $9 \%, 12 \%$ and $88 \%$, respectively, compared to the deeplypipelined decoder. Also on FPGA, with a throughput over $50 \mathrm{Gbps}$, using an initiation interval as small as 4 removes the need for any SRAM blocks, while the usage of LUTs and registers decreases by $20 \%$ and $23 \%$, respectively. Finally, from Table 5.1, if a coded throughput of $1.5 \mathrm{Gbps}$ is sufficient for the application, $I=167$ will result in savings of 32\%, 77\% and $100 \%$ in terms of LUTs, registers and RAM bits, compared to the deeply-pipelined architecture $(I=1)$. On ASIC, the area is largely dominated by registers and thus increasing the initiation interval has great effect on the total area as shown in Table 5.2. For example, using $\mathcal{I}=50$ results in an area that is more than 12 times smaller, at the cost of a throughput that is 50 times lower.

As expected, increasing the initiation interval $\mathcal{I}$ offers a diminishing return as it gets closer to 
Table 5.1: Decoders for a $(1024,512)$ polar code with various initiation interval $\mathcal{I}$ implemented on an FPGA.

\begin{tabular}{crrrrrr}
\hline $\mathcal{I}$ & \multirow{2}{*}{ LUTs } & \multirow{2}{*}{ Regs. } & $\begin{array}{r}\text { RAM } \\
(\mathrm{kbits})\end{array}$ & $\begin{array}{c}f \\
(\mathrm{MHz})\end{array}$ & $\begin{array}{c}\text { T/P } \\
(\mathrm{Gbps})\end{array}$ & $\begin{array}{c}\text { Latency } \\
(\mu \mathrm{s})\end{array}$ \\
\hline 1 & 136,874 & 188,071 & 83.92 & 248 & 254.1 & 1.47 \\
2 & 124,532 & 166,361 & 9.97 & 238 & 121.7 & 1.53 \\
3 & 114,173 & 152,182 & 4.68 & 208 & 71.1 & 1.75 \\
4 & 110,381 & 145,000 & 0 & 203 & 52.1 & 1.79 \\
50 & 86,998 & 65,618 & 0 & 218 & 4.5 & 1.67 \\
167 & 93,225 & 43,236 & 0 & 239 & 1.5 & 1.52 \\
\hline
\end{tabular}

Table 5.2: Decoders for a $(1024,512)$ polar code with various initiation interval $\mathcal{I}$ implemented on an ASIC.

\begin{tabular}{cccrrr}
\hline $\mathcal{I}$ & $\begin{array}{c}\text { Tot. Area } \\
\left(\mathrm{mm}^{2}\right)\end{array}$ & $\begin{array}{c}\text { Mem. Area } \\
\left(\mathrm{mm}^{2}\right)\end{array}$ & $\begin{array}{c}f \\
(\mathrm{MHz})\end{array}$ & $\begin{array}{r}\mathrm{T} / \mathrm{P} \\
(\mathrm{Gbps})\end{array}$ & $\begin{array}{c}\text { Latency } \\
(\mu \mathrm{s})\end{array}$ \\
\hline 1 & 4.627 & 3.911 & 1245 & $1,274.9$ & 0.29 \\
2 & 3.326 & 3.150 & 1020 & 522.2 & 0.36 \\
3 & 2.314 & 2.138 & 1005 & 343.0 & 0.36 \\
4 & 1.665 & 1.063 & 1003 & 256.8 & 0.36 \\
50 & 0.366 & 0.143 & 1003 & 20.5 & 0.36 \\
167 & 0.289 & 0.089 & 1003 & 6.2 & 0.36 \\
\hline
\end{tabular}

the maximum of 167. Table 5.1 also shows that on FPGA increasing $\mathcal{I}$ first reduces the maximum execution frequency but, eventually, it reincreases almost back to the value it had with $\mathcal{I}=1$. Inspection of the critical paths reveals that this frequency increase is a result of shorter wire delays. As the number of LUTs and registers decreases with an increasing $\mathcal{I}$, at some point, it becomes easier to use resources that are close to each other.

\subsubsection{Comparison with State-of-the-Art Decoders}

In this section, we compare our work with that of the fastest state-of-art polar decoder implementations: [15], [17], [47]. The work of [17] was presented in Chapter 3. In [15], ASIC results are provided. The work of [17] and [47] provide results for both ASIC and FPGA implementations. The BP decoder of [15] is an iterative decoder utilizing early termination to improve the 
average throughput. However, as it does not include the necessary buffers to accommodate that functionality, we add the sustainable throughput for consistency.

Table 5.3 shows that regardless of the implementation technology, our family of architectures can deliver from one to three orders of magnitude greater coded throughput. On ASIC, the latency is more than 10 times lower than that of [15] and about the same as both [17] and [47].

Table 5.3: Comparison with state-of-the-art polar decoders.

\begin{tabular}{|c|c|c|c|c|c|c|c|c|c|}
\hline & \multicolumn{6}{|c|}{ This work } & [17] & {$[15]^{\diamond}$} & {$[47]$} \\
\hline Algorithm & \multirow{2}{*}{\multicolumn{6}{|c|}{$\begin{array}{c}\text { Fast-SSC } \\
(1024,512)\end{array}$}} & Fast-SSC & $\mathrm{BP}$ & $\mathrm{SC}$ \\
\hline Code & & & & & & & $(1024,512)$ & $(1024,512)$ & $(1024, k)$ \\
\hline IC Type & \multicolumn{3}{|c|}{ FPGA } & \multicolumn{3}{|c|}{ ASIC } & ASIC & ASIC & ASIC \\
\hline Technology & \multicolumn{3}{|c|}{$40 \mathrm{~nm}$} & \multicolumn{3}{|c|}{$28 \mathrm{~nm}$} & $65 \mathrm{~nm}$ & $65 \mathrm{~nm}$ & $90 \mathrm{~nm}$ \\
\hline Init. Interval $(\mathcal{I})$ & 167 & 50 & 1 & 167 & 50 & 1 & - & - & - \\
\hline Supply (V) & 0.9 & 0.9 & 0.9 & 1.0 & 1.0 & 1.0 & 1.0 & 1.0 & 1.3 \\
\hline Area $\left(\mathrm{mm}^{2}\right)$ & - & - & - & 0.29 & 0.37 & 4.63 & 0.69 & 1.48 & 3.21 \\
\hline Frequency $(\mathrm{MHz})$ & 239 & 218 & 248 & 1003 & 1003 & 1245 & 600 & 300 & 2.5 \\
\hline Latency $(\mu \mathrm{s})$ & 1.5 & 1.7 & 1.5 & 0.4 & 0.4 & 0.3 & 0.4 & 50 & 0.4 \\
\hline $\mathbf{T} / \mathbf{P}$ (Gbps) & 1.5 & 4.5 & 254 & 6.2 & 20.5 & 1275 & 3.7 & $4.7 @ 4 \mathrm{~dB}$ & 2.9 \\
\hline Sust. T/P (Gbps) & 1.5 & 4.5 & 254 & 6.2 & 20.5 & 1275 & 3.7 & 2.0 & 2.9 \\
\hline Area Eff. $\left(\mathrm{Gbps} / \mathrm{mm}^{2}\right)$ & - & - & - & 21.45 & 56.01 & 275.53 & 5.4 & $3.18 @ 4 \mathrm{~dB}$ & 0.8 \\
\hline Power $(\mathrm{mW})$ & 1420 & 1454 & 5532 & 169 & 271 & 8793 & 215 & 478 & 191 \\
\hline Energy (pJ/bit) & 946.7 & 323.2 & 21.8 & 27.3 & 13.2 & 6.9 & 57.5 & 102.1 & 74.5 \\
\hline \multicolumn{10}{|c|}{ Normalized results for $28 \mathrm{~nm}$ and $1.0 \mathrm{~V}$. } \\
\hline Area $\left(\mathrm{mm}^{2}\right)$ & - & - & - & 0.29 & 0.37 & 4.63 & 0.13 & 0.27 & 0.31 \\
\hline Frequency $(\mathrm{MHz})$ & 341 & 311 & 354 & 1003 & 1003 & 1245 & 1392 & 696 & 8.0 \\
\hline Latency $(\mu \mathrm{s})$ & 1.1 & 1.2 & 1.1 & 0.4 & 0.4 & 0.3 & 0.2 & 21.5 & 0.1 \\
\hline Sust. T/P (Gbps) & 2.1 & 6.4 & 363 & 6.2 & 20.5 & 1275 & 8.6 & 4.6 & 9.2 \\
\hline Area Eff. $\left(\mathrm{Gbps} / \mathrm{mm}^{2}\right)$ & - & - & - & 21.45 & 56.01 & 275.53 & 66.2 & 17.2 & 29.6 \\
\hline Power $(\mathrm{mW})$ & 1227 & 1257 & 4781 & 169 & 271 & 8793 & 93 & 206 & 35 \\
\hline Energy (pJ/bit) & 818.1 & 279.2 & 18.8 & 27.3 & 13.2 & 6.9 & 25.0 & 44.8 & 3.8 \\
\hline
\end{tabular}

$\diamond$ Measurement results.

Normalizing the results of [15], [17], [47] to $28 \mathrm{~nm}$ CMOS technology with a supply voltage of $1.0 \mathrm{~V}$, the coded throughput is still one or two orders of magnitude greater. Latency is two orders of magnitude lower than [15] but approximately 3 to 4 times greater than [47]. Looking at the energy efficiency for the deeply-pipelined architecture on ASIC, the proposed decoder is 6 times more efficient than [15], 3.5 times more efficient than [17] and about 2 times less efficient than 
Table 5.4: Comparison with other FPGA implementations.

\begin{tabular}{crrrccc}
\hline Impl. & LUTs & Regs. & $\begin{array}{r}\text { RAM } \\
(\mathrm{kbits})\end{array}$ & $\begin{array}{c}f \\
(\mathrm{MHz})\end{array}$ & $\begin{array}{c}\mathrm{T} / \mathrm{P} \\
(\mathrm{Gbps})\end{array}$ & $\begin{array}{c}\text { Latency } \\
(\mu \mathrm{s})\end{array}$ \\
\hline This work & 93,225 & 43,236 & 0 & 239 & 1.5 & 1.5 \\
{$[17]$} & 23,353 & 5,814 & 44 & 103 & 0.6 & 1.6 \\
{$[47] \dagger$} & 193,456 & 6,151 & N/A & 1 & 0.6 & 3.4 \\
\hline
\end{tabular}

[47]. In terms of area efficiency, the same decoder is 4.2, 16 and 9.4 times more efficient than that of [17], [15] and [47], respectively.

Table 5.4 compares our proposed fully-unrolled partially-pipelined architecture, with the maximum initiation interval $\mathcal{I}_{\max }=167$, against the fastest FPGA implementations of [17], [47]. The work of [47] is marked with ( $\dagger$ ) as these results are for a different FPGA, the Xilinx Virtex-6 XC6VLX550T. Note however that this Xilinx FPGA is implemented in $40 \mathrm{~nm}$ CMOS technology and features 6-input LUTs, like the Altera Stratix IV FPGA.

It can be seen that a decoder built with one of our proposed architectures can achieve nearly 3 times the throughput of both [17] and [47] with a slightly lower latency. In terms of resources, compared to the decoder of [17], our decoder requires almost 4 and 7 times the number of LUTs and registers, respectively. Note however that we do not require any RAM for the proposed implementation, while the other decoder from Chapter 3 uses 44 kbits. Compared to the SC decoder of [47], our decoder requires less than half the LUTs, but needs more than 7 times the number of registers. It should be noted that the decoder of [47] does not contain the necessary memory to load the next frame while a frame is being decoded, nor the necessary memory to offload the previously estimated codeword as decoding is taking place.

\subsubsection{Effect of the Code Length and Rate}

Results for other polar codes are presented in this section where we show the effect of the code length and rate on performance and resource usage.

Tables 5.5, 5.6, 5.7 and 5.7 show the effect of the code length on resource usage, coded throughput, and decoding latency for polar codes of short to moderate lengths. Tables 5.5 and 5.6 contain results for the fully-unrolled deeply-pipelined architecture $(\mathcal{I}=1)$ and the code rate $R$ is fixed to $1 / 2$ for all polar codes. Tables 5.7 and 5.8 contain results for the fully-unrolled partially-pipelined 
Table 5.5: Deeply-pipelined decoders for polar codes of various lengths with rate $R=1 / 2$ implemented on an FPGA.

\begin{tabular}{crrrcrc}
\hline$N$ & LUTs & Regs. & $\begin{array}{c}\text { RAM } \\
(\mathrm{kbits})\end{array}$ & $\begin{array}{c}f \\
(\mathrm{MHz})\end{array}$ & $\begin{array}{c}\text { T/P } \\
(\mathrm{Gbps})\end{array}$ & $\begin{array}{c}\text { Latency } \\
(\mu \mathrm{s})\end{array}$ \\
\hline 128 & 9,917 & 18,543 & 0 & 357 & 45.7 & 0.21 \\
256 & 27,734 & 44,010 & 0 & 324 & 83.0 & 0.41 \\
512 & 64,723 & 105,687 & 4 & 275 & 141.1 & 0.74 \\
1024 & 136,874 & 188,071 & 84 & 248 & 254.1 & 1.47 \\
2048 & 217,175 & 261,112 & 5,362 & 203 & 415.7 & 3.21 \\
\hline
\end{tabular}

Table 5.6: Deeply-pipelined decoders for polar codes of various lengths with rate $R=1 / 2$ implemented on an ASIC.

\begin{tabular}{cccccrr}
\hline$N$ & $\begin{array}{c}\text { Tot. Area } \\
\left(\mathrm{mm}^{2}\right)\end{array}$ & $\begin{array}{c}\text { Log. Area } \\
\left(\mathrm{mm}^{2}\right)\end{array}$ & $\begin{array}{c}\text { Mem. Area } \\
\left(\mathrm{mm}^{2}\right)\end{array}$ & $\begin{array}{c}f \\
(\mathrm{MHz})\end{array}$ & $\begin{array}{c}\mathrm{T} / \mathrm{P} \\
(\mathrm{Gbps})\end{array}$ & $\begin{array}{c}\text { Latency } \\
(\mathrm{ns})\end{array}$ \\
\hline 128 & 0.125 & 0.027 & 0.098 & 1383 & 177.0 & 55.0 \\
256 & 0.412 & 0.079 & 0.332 & 1353 & 346.4 & 99.0 \\
512 & 1.263 & 0.217 & 1.045 & 1328 & 679.9 & 156.6 \\
1024 & 4.627 & 0.715 & 3.911 & 1245 & $1,274.9$ & 292.4 \\
\hline
\end{tabular}

architecture where the maximum initiation interval $\left(\mathcal{I}_{\max }\right)$ is used and the code rate $R$ is fixed to $5 / 6$.

As shown in Tables 5.5 and 5.6, with a deeply-pipelined architecture, both the logic usage and memory requirements are close to being quadratic in code length $N$.

On FPGAs, the decoders for the three longest codes of Table 5.5 are capable of a coded throughput greater than 100 Gbps. Notably, the $N=2048$ code reaches 400 Gbps. On ASIC, a throughput exceeding 1 Tbps can be achieved with a decoder for a polar code of length $N=1024$ as shown in Table 5.6. The decoder for the $(2048,1024)$ polar code could not be synthesized for ASIC on our server due to insufficient memory.

Table 5.7 shows that for a partially-pipelined decoder where the initiation interval is set to $\mathcal{I}_{\max }$, it is possible to fit a code of length $N=4096$ on the Stratix IV GX 530. The amount of RAM required is not illustrated in the table as none of the decoders are using any of the available RAM. Also note that no LUTs are used as memory. In other words, for pipelined decoders using $\mathcal{I}_{\max }$ as the initiation interval, registers are the only memory resources needed. Table 5.7 also shows that these maximum initiation intervals lead to a much more modest throughput. In the case of 
Table 5.7: Partially-pipelined decoders with initiation interval set to $\mathcal{I}_{\max }$ for polar codes of various lengths with rate $R=5 / 6$ implemented on an FPGA.

\begin{tabular}{ccrrccc}
\hline$N$ & $\mathcal{I}$ & LUTs & Regs. & $\begin{array}{c}f \\
(\mathrm{MHz})\end{array}$ & $\begin{array}{c}\mathrm{T} / \mathrm{P} \\
(\mathrm{Gbps})\end{array}$ & $\begin{array}{c}\text { Latency } \\
(\mu \mathrm{s})\end{array}$ \\
\hline 1024 & 206 & 75,895 & 42,026 & 236 & 1.17 & 1.11 \\
2048 & 338 & 165,329 & 75,678 & 220 & 1.33 & 2.02 \\
4096 & 665 & 364,320 & 172,909 & 123 & 0.76 & 7.04 \\
\hline
\end{tabular}

Table 5.8: Partially-pipelined decoders with initiation interval set to $\mathcal{I}_{\max }$ for polar codes of various lengths with rate $R=5 / 6$ implemented on an ASIC clocked at $1 \mathrm{GHz}$.

\begin{tabular}{ccccccc}
\hline$N$ & $\mathcal{I}$ & $\begin{array}{c}\text { Tot. Area } \\
\left(\mathrm{mm}^{2}\right)\end{array}$ & $\begin{array}{c}\text { Log. Area } \\
\left(\mathrm{mm}^{2}\right)\end{array}$ & $\begin{array}{c}\text { Mem. Area } \\
\left(\mathrm{mm}^{2}\right)\end{array}$ & $\begin{array}{c}\mathrm{T} / \mathrm{P} \\
(\mathrm{Gbps})\end{array}$ & $\begin{array}{c}\text { Latency } \\
(\mu \mathrm{s})\end{array}$ \\
\hline 1024 & 206 & 0.230 & 0.169 & 0.070 & 5.0 & 0.26 \\
2048 & 338 & 0.509 & 0.345 & 0.164 & 6.1 & 0.44 \\
4096 & 665 & 1.192 & 0.820 & 0.372 & 6.2 & 0.87 \\
\hline
\end{tabular}

the $(4096,3413)$ polar code, we can see a major latency increase compared to the shorter codes. This latency increase can be explained by the maximum clock frequency drop which in turn can be explained by the fact that $94 \%$ of the total available logic resources in that FPGA were required to implement this decoder.

At some point on FPGAs, a fully-unrolled architecture is no longer advantageous over a more compact architecture like the one of [8]. With the Stratix IV GX 530 as an FPGA target, a fullyunrolled decoder for a polar code of length $N=4096$ is too complex to provide good throughput and latency. Even with the maximum initiation interval, $94 \%$ of the logic resources are required for a coded throughput under 1 Gbps. By comparison, a decoder built with the architecture of [8] would result in a coded throughput in the vicinity of $1 \mathrm{Gbps}$ at $110 \mathrm{MHz}$. Targeting a more recent FPGA could lead to different results and conclusions.

On ASIC, both the memory and total area scale linearly with $N$ for a partially-pipelined architecture with $\mathcal{I}_{\max }$. The results of Table 5.8 also show that it was possible to synthesize ASIC decoders for larger code lengths than what was possible with a deeply-pipelined architecture.

The effect of using different code rates for a polar code of length $N=1024$ is shown in Tables 5.9 and 5.10. We note that the higher-rate codes do not have noticeably lower latency 
Table 5.9: Deeply-pipelined decoders for polar codes of length $N=1024$ with common rates implemented on an FPGA.

\begin{tabular}{|c|c|c|c|c|c|c|c|}
\hline \multirow[b]{2}{*}{$R$} & \multirow[b]{2}{*}{ LUTs } & \multirow[b]{2}{*}{ Regs. } & \multirow{2}{*}{$\underset{\text { (bits) }}{\text { RAM }}$} & \multirow{2}{*}{$\begin{array}{c}f \\
(\mathrm{MHz})\end{array}$} & \multirow{2}{*}{$\begin{array}{c}\mathrm{T} / \mathrm{P} \\
\text { (Gbps) }\end{array}$} & \multicolumn{2}{|c|}{ Latency } \\
\hline & & & & & & (CCs) & $(\mu \mathrm{s})$ \\
\hline $1 / 2$ & 136,874 & 188,071 & 83,924 & 248 & 254.1 & 364 & 1.47 \\
\hline $2 / 3$ & 137,230 & 183,957 & 73,020 & 250 & 256.0 & 326 & 1.30 \\
\hline $3 / 4$ & 151,282 & 204,479 & 83,288 & 227 & 232.7 & 373 & 1.64 \\
\hline $5 / 6$ & 145,659 & 198,876 & 82,584 & 229 & 234.4 & 323 & 1.41 \\
\hline
\end{tabular}

Table 5.10: Deeply-pipelined decoders for polar codes of length $N=1024$ with common rates implemented on an ASIC.

\begin{tabular}{lccccccc}
\hline & & & & & & & \multicolumn{2}{c}{ Latency } \\
\cline { 7 - 8 }$R$ & $\begin{array}{c}\text { Tot. Area } \\
\left(\mathrm{mm}^{2}\right)\end{array}$ & $\begin{array}{c}\text { Log. Area } \\
\left(\mathrm{mm}^{2}\right)\end{array}$ & $\begin{array}{c}\text { Mem. Area } \\
\left(\mathrm{mm}^{2}\right)\end{array}$ & $\begin{array}{c}f \\
(\mathrm{MHz})\end{array}$ & $\begin{array}{c}\mathrm{T} / \mathrm{P} \\
\mathrm{Gbps})\end{array}$ & $(\mathrm{CCs})$ & $(\mathrm{ns})$ \\
\hline $1 / 2$ & 4.627 & 0.715 & 3.911 & 1245 & $1,274.9$ & 364 & 292.4 \\
$2 / 3$ & 4.896 & 0.740 & 4.156 & 1300 & $1,331.2$ & 326 & 250.8 \\
$3 / 4$ & 5.895 & 0.872 & 5.023 & 1245 & $1,274.9$ & 373 & 299.6 \\
$5 / 6$ & 5.511 & 0.816 & 4.694 & 1361 & $1,393.7$ & 323 & 237.3 \\
\hline
\end{tabular}

compared to the rate- $1 / 2$ code, contrary to what was observed in [8]. This is due to limiting the width of SPC nodes to 4 in this work, whereas it was left unbounded in [8], [16], [17]. The result is that long SPC codes are implemented as trees whose left-most child is a width-4 SPC node and the others are all rate- 1 nodes. Thus, for each additional stage $\left(\log _{2} N_{v}-\log _{2} N_{\mathrm{SPC}}\right)$ of an SPC code of length $N_{v}>N_{\mathrm{SPC}}$, four nodes with a total latency of $3 \mathrm{CCs}$ are required: $F, G$ followed by $I$, and Combine. This brings the total latency of decoding a long SPC code to $3\left(\log _{2} N_{v}-\log _{2} N_{\mathrm{SPC}}\right)+1$ CCs compared to $\left\lceil N_{v} / \mathcal{P}\right\rceil+4$ in [8], where $\mathcal{P}$ is the number of LLRs that can be read simultaneously (256 was a typical value for $\mathcal{P}$ in [8]).

Fig. 5.7 gives a graphical overview of the maximum resource usage requirements on FPGA for a given achievable coded throughput. The fully-unrolled deeply- and partially-pipelined decoders were taken from Tables 5.1 and 5.5, respectively. The resynthesized polar decoder of [8] is also included for reference. The red asterisks show that with a deeply-pipelined decoder architecture (initiation interval $\mathcal{I}=1$ ), the coded throughput increases at a higher rate than the maximum resource usage as the code length $N$ increases. The blue diamonds illustrate the effect of various 


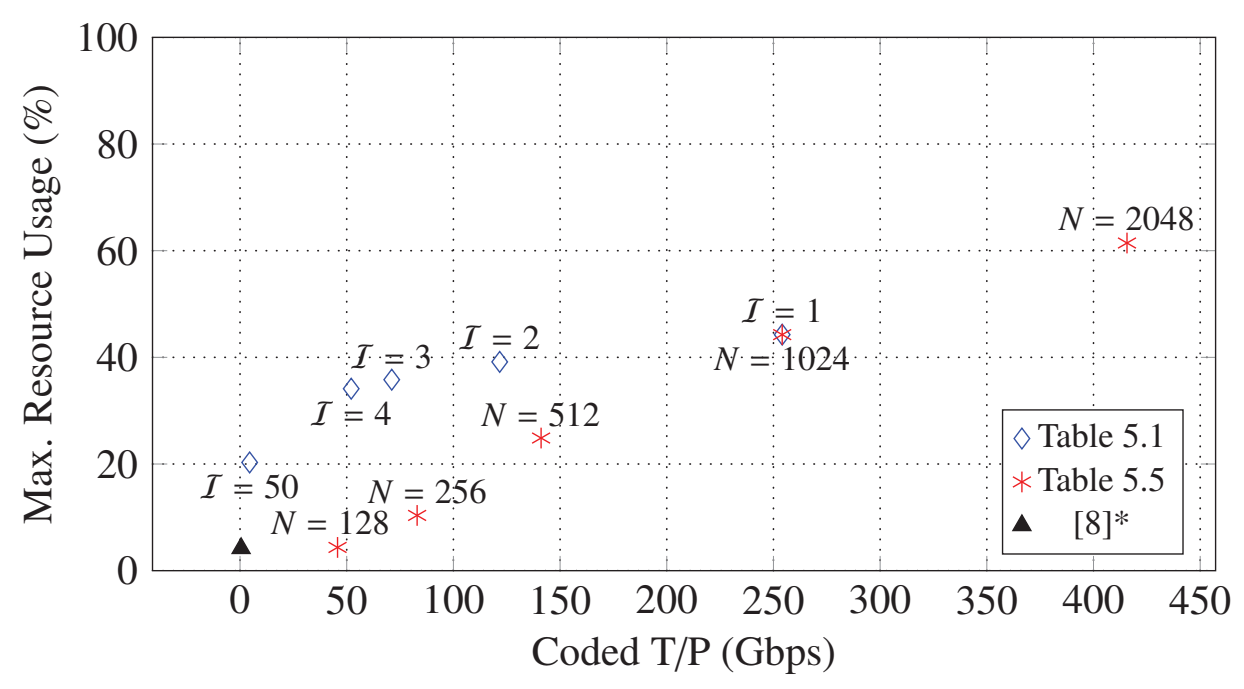

Figure 5.7: Overview of the maximum FPGA resource usage and coded throughput for some partially-pipelined (Table 5.1) and deeply-pipelined (Table 5.5) polar decoders. The resynthesized polar decoder of [8] is also included for reference.

initiation intervals for the same $(1024,512)$ polar code. We see that decreasing $\mathcal{I}$ leads to increasingly interesting implementation alternatives, as the gains in throughput are obtained at the expense of a smaller increase in the maximum resource usage. When it can be afforded and that the FPGA input data rate is sufficient, the extra $2.9 \%$ in maximum resource usage allows doubling the throughput, from $\mathcal{I}=2$ to $\mathcal{I}=1$.

\subsubsection{On the Use of Code Shortening in an Unrolled Decoder}

Code shortening is a well-known technique used to create a rate- and length-flexible error-correction system. Multiple such schemes were proposed for use with polar codes [24], [66], [67]. The technique presented in [24] could be used with this work. It starts from a systematic $(N, k)$ polar code and shortens it by $h$ bits, resulting in a $\left(N_{s}, k_{s}\right)$ polar code, where $N_{s}=N-h$ and $k_{s}=k-h$. At the encoder, the $h$ information bit locations of highest indices are set to a predetermined value (usually 0 ). After encoding, these bits are discarded from $\boldsymbol{x}$ before being transmitted over the channel. At the decoder, the corresponding soft-inputs channel values are set to a certain 0 or 1 , depending on the predetermined value used at the encoder.

To support a shortened $(N-h, k-h)$ polar code, an unrolled decoder requires additional circuitry at the input to insert LLR values for the discarded bits - the maximum LLR value when the 
discarded bits are assumed to be 0 . Since, the scheme of [24] chooses the information bits with largest indices to discard, the routing overhead would be minor.

\subsubsection{I/O Bounded Decoding}

The family of architectures that we propose requires tremendous throughput at the input of the decoder, especially with a deeply-pipelined architecture. For example, if a quantization of $Q_{c}=4$ bits is used for channel LLRs, for every estimated bit, 4 times as many bits have to be loaded into the decoder. In other words, the total data rate is 5 times that of the output. This can be a significant challenge on both FPGAs and ASICs.

On FPGA, if 38 of the 48 high-speed transceivers (approximately $4 / 5$ ) featured on a Stratix IV GX are to be used to load the channel LLRs and the remainder to output the estimated codewords, the maximum theoretical input data rate achievable will be of $323 \mathrm{Gbps}$. On the more recent Stratix V GX, using 53 of the 66 transceivers at their peak data rate of 14.1 Gbps sums up to 747 Gbps available for input. However, the fully-unrolled deeply-pipelined $(1024,512)$ and $(2048,1024)$ polar decoders discussed above require an input data rate that is over 1 Tbps.

If only for that reason, partially-pipelined architectures are certainly more attractive, at least using current FPGA technology. Notice however that data rates in the vicinity of 1 Tbps are expected to be reachable in the incoming Xilinx UltraScale [68] and Altera Generation 10 [69] families of FPGAs. On ASICs, the number of high-speed transceivers is not fixed and a custom solution can be built.

\subsection{Conclusion}

In this chapter we presented a new family of architectures for fully-unrolled polar decoders. With an initiation interval that can be ajusted, these architectures make it possible to find a trade-off between area (or resource usage) and achievable throughput without affecting decoding latency. We showed that a fully-unrolled deeply-pipelined decoder implemented on an FPGA can achieve a throughput greater than $400 \mathrm{Gbps}$, which is two orders of magnitude greater than state-of-theart polar decoders while maintaining a good latency. On ASICs, we showed that the proposed fully-unrolled deeply-pipelined decoders could achieve a throughput that would be two or three orders of magnitude greater than the state-of-the-art decoders with an order of magnitude better normalized area efficiency and a competitive energy efficiency. One of the proposed decoder has a 
coded throughput in excess of 1 Tbps at $6.9 \mathrm{pJ} /$ bit on ASICs. We believe that these architectures make polar codes a promising candidate for future $5 \mathrm{G}$ communications. 


\section{Chapter 6}

\section{Multi-mode Unrolled Polar Decoding}

Unrolled decoders are architectures that provide the greatest decoding speed, by orders of magnitude compared to their more compact counterparts. However, unrolled decoders are built for a specific, fixed, code. In this chapter, we present a new method to enable the use of multiple code lengths and rates in a fully-unrolled polar decoder architecture. This novel method leads to a length- and rate-flexible decoder while retaining the very high speed typical to those decoders. We present results for two versions of a multi-mode decoder supporting eight and ten different polar codes, respectively. Both are capable of a peak throughput of $25.6 \mathrm{Gbps}$. For each decoder, the energy efficiency for the longest supported polar code is shown to be of $14.8 \mathrm{pJ} / \mathrm{bit}$ at $250 \mathrm{MHz}$ and of $8.8 \mathrm{pJ} / \mathrm{bit}$ at $500 \mathrm{MHz}$ on an ASIC built in $65 \mathrm{~nm}$ CMOS technology.

\subsection{Introduction}

In the previous chapter (and in [21]), unrolled hardware architectures for polar decoders were proposed. Results showed a very high throughput, greater than 1 Tbps. However, these architectures are built for a fixed polar code i.e. the code length or rate cannot be modified at execution time. This is a major drawback for most modern wireless communication applications that largely benefit from the support of multiple code lengths and rates.

The goal of this chapter is to show how an unrolled decoder built specifically for a polar code, of fixed length and rate, can be transformed into a multi-mode decoder supporting many codes of various lengths and rates. More specifically, we show how decoders for moderate-length polar codes contain decoders for many other shorter_but practical_-polar codes of both high and low 
rates. The required hardware modifications are detailed, and ASIC synthesis and power estimations are provided for the $65 \mathrm{~nm}$ CMOS technology from TSMC. Results show a peak information throughput greater than $25 \mathrm{Gbps}$ at $250 \mathrm{MHz}$ in $4.29 \mathrm{~mm}^{2}$ or at $500 \mathrm{MHz}$ in $1.71 \mathrm{~mm}^{2}$. Latency is of $2 \mu \mathrm{s}$ and $650 \mathrm{~ns}$ for the former and latter.

The remainder of this chapter starts with Sections 6.2 and 6.3 where a supporting decoder tree example is provided along with its unrolled hardware implementation. Section 6.4 then explains the concept, hardware modifications and other practical considerations related to the proposed multi-mode decoder. Error-correction performance and implementation results are provided in Section 6.5. Comparison against the fastest state-of-the-art polar decoder implementations in the literature is carried out in Section 6.5 as well. Finally, a conclusion is drawn in Section 6.6.

\subsection{Polar Code Example and its Decoder Tree Representations}

Fig. 6.1a illustrates the decoder tree for a $(16,12)$ polar code, where black and white nodes are information and frozen bits, respectively. The Left-Hand-Side (LHS) and Right-Hand-Side (RHS) subtrees rooted in the top node are polar codes of length $N / 2$. In the remainder of this chapter, we designate the polar code, of length $N$, decoded by traversing the whole decoder tree as the master code and the various codes of lengths smaller than $N$ as constituent codes.

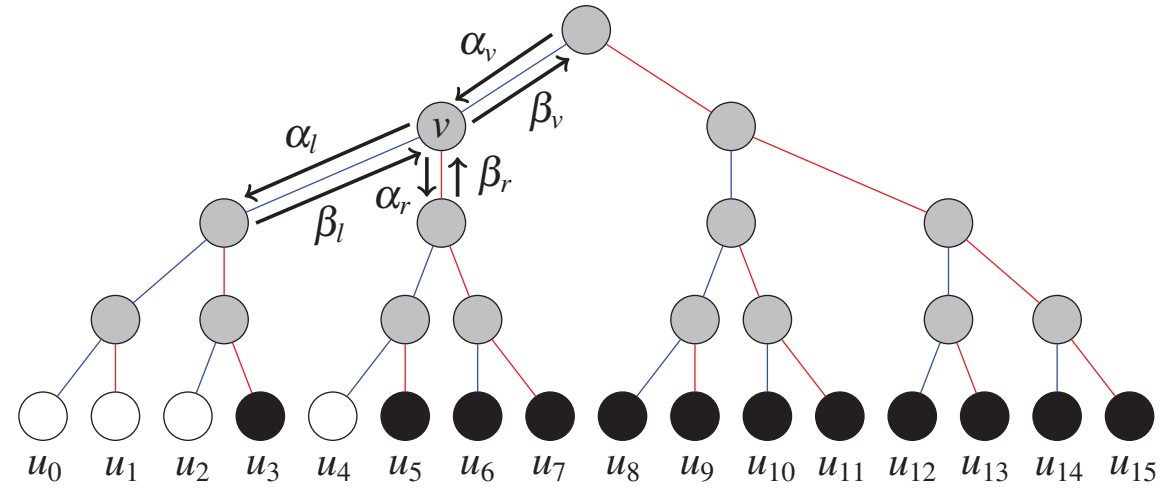

(a) SC

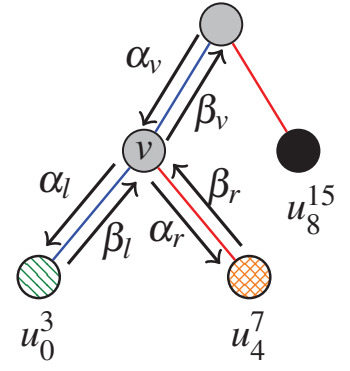

(b) Fast-SSC

Figure 6.1: Decoder trees for SC (a) and Fast-SSC (b) decoding of a $(16,12)$ polar code. 


\subsection{Unrolled Architectures}

In an unrolled decoder, each and every operation required is instantiated so that data can flow through the decoder with minimal control. Unrolled architectures for polar decoders are described in depth in Chapter 5.

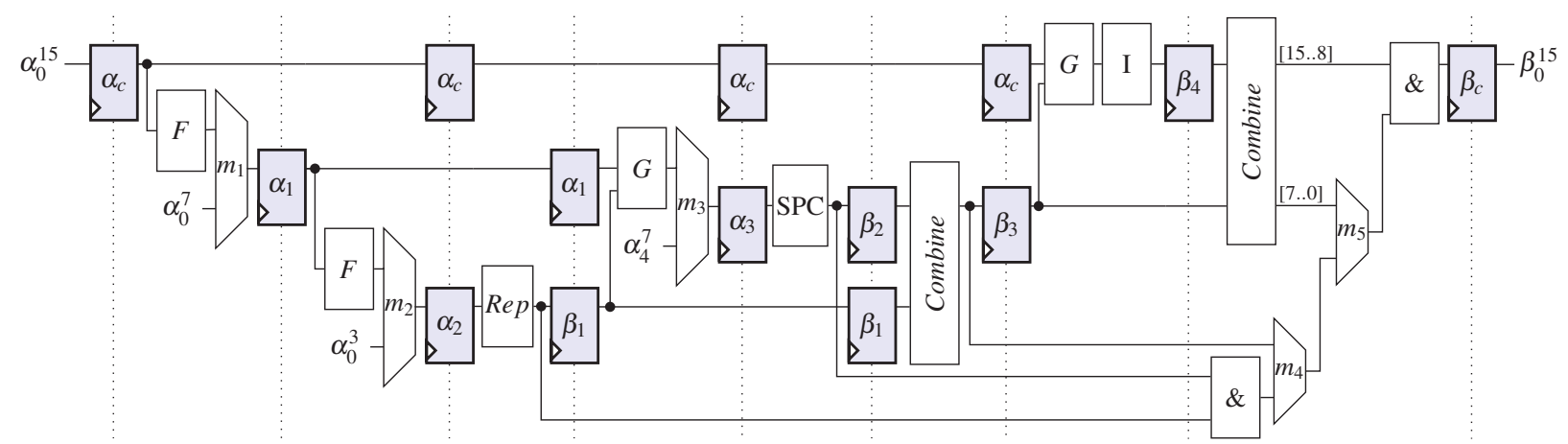

Figure 6.2: Unrolled partially-pipelined decoder for a $(16,12)$ polar code with initiation interval $\mathcal{I}=2$. Clock, flip-flop enable and multiplexer select signals are omitted for clarity.

Fig. 6.2 shows a fully-unrolled partially-pipelined decoder [21] with an initiation interval $\mathcal{I}=2$ for the $(16,12)$ polar code of Fig. $6.1 \mathrm{~b}$ - the initiation interval can be seen as the minimum number of clock cycles between two codeword estimates. Some control and routing logic was added to make it multi-mode as proposed in this chapter, details are provided in the next section. The $\alpha$ and $\beta$ blocks illustrated in light blue are registers storing LLRs or bit estimates, respectively. White blocks are Fast-SSC functions as detailed in Section 2.6, with the exception of the "\&" blocks that are concatenation operators.

\subsection{Multi-mode Unrolled Decoders}

It can be noted that an unrolled decoder for a polar code of length $N$ is composed of unrolled decoders for two polar codes of length $N / 2$, which are each composed of unrolled decoders for two polar codes of length $N / 4$, and so on. Thus, by adding some control and routing logic, it is possible to directly feed and read data from the unrolled decoders for subcodes of length smaller than $N$. The end result is a multi-mode decoder supporting frames of various lengths and code rates. 


\subsubsection{Hardware Modifications to the Unrolled Decoders}

Consider the decoder tree shown in Fig. 6.1b along with its unrolled implementation as illustrated in Fig. 6.2. In Fig. 6.1b, the constituent code taking root in $v$ is an $(8,4)$ polar code. Its corresponding decoder can be directly employed by placing the 8 channels LLRs into $\alpha_{0}^{7}$ and by selecting the bottom input of the multiplexer $m_{1}$ illustrated in Fig. 6.2. Its estimated codeword is retrieved from reading the output of the Combine block feeding the $\beta_{4}$ register i.e. by selecting the top and bottom inputs from $m_{4}$ and $m_{5}$, respectively, and by reading the 8 least-significant bits from $\beta_{0}^{15}$. Similarly, still in Fig. 6.2, the decoders for the repetition and SPC constituent codes can be fed via the $m_{2}$ and $m_{3}$ multiplexers and their output eventually recovered from the output of the Rep and SPC blocks, respectively.

Although not illustrated in Fig. 6.2, the unrolled decoders proposed in the previous chapter feature a minimal controller. As described at the end of Section 5.3.3, its main task is twofold. First, it generates a done signal to indicate that a new estimated codeword is available at the output. Second, in the case of a partially-pipelined decoder i.e. with an initiation interval $\mathcal{I}$ greater than 1 like in Fig. 6.2, it asserts the various flip-flop enable signals at the correct time. Both are accomplished using a counter, albeit independently.

While not mandatory, the functionality of these counters is altered to better accommodate the use of multiple polar codes. Two LUTs are added. One LUT stores the decoding latency, in CCs, of each code. It serves as a stopping criteria to generate the done signal. The other LUT stores the clock cycle "value" $i_{\text {start }}$ at which the enable-signal generator circuit should start. Each non-master code may start at a value $\left(i_{\text {start }} \bmod \mathcal{I}\right) \neq 0$. In such cases, using the unaltered controller would result in the waste of $\left(i_{\text {start }} \bmod I\right)$ CCs. It can be significant for short codes, especially with large values of $\mathcal{I}$. For example, without these changes, for the implementation with a master code of length 1024 and $\mathcal{I}=20$ presented in Section 6.5 below, the latency for the $(128,96)$ polar code would increase by $20 \%$ as $\left(i_{\text {start }} \bmod \mathcal{I}\right)=17$ and the decoding latency is of $82 \mathrm{CCs}$.

Lastly, the modified controller also generates the multiplexer select signals, allowing proper data routing, based on the selected mode.

\subsubsection{On the Construction of the Master Code}

Conventional approaches construct polar codes for a given channel type and condition. In this work, many of the constituent codes contained within a master code are not only used internally 
to detect and correct errors, they are used separately as well. Therefore, we propose to assemble a master code using two optimized constituent codes in order to increase the number of optimized polar codes available. Doing so, the number of information bits, or the code rate, of the second largest supported codes can be selected. In the following, a master code of length 2048 is constructed by concatenating two constituent codes of length 1024. The LHS and RHS constituent codes are chosen to have a rate of $1 / 2$ and of $5 / 6$, respectively. As a result, the assembled master code has rate $2 / 3$. The location of the frozen bits in the master code is dictated by its constituent codes. Note that the constituent code with the lowest rate is put on the left-and the one with the highest rate on the right - to minimize the coding loss associated with a non-optimized polar code.

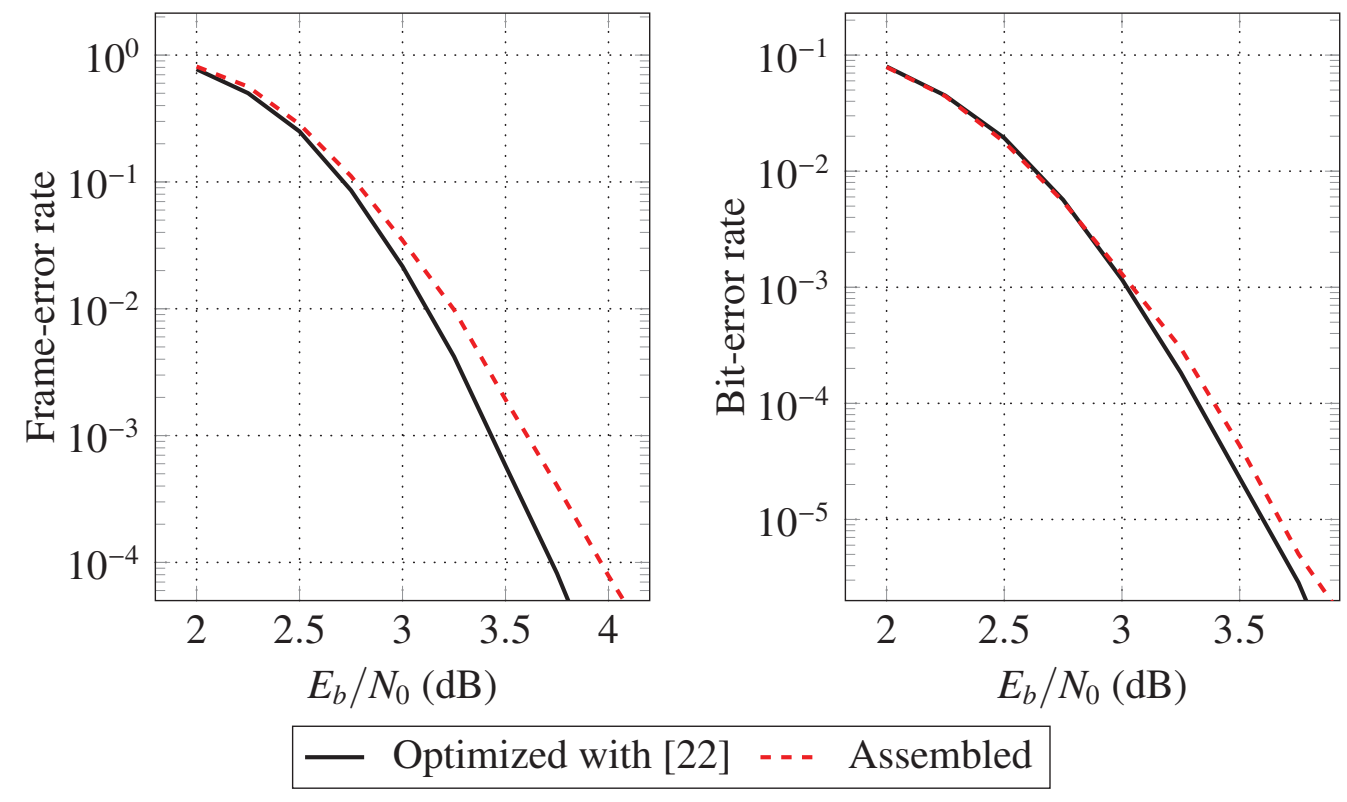

Figure 6.3: Error-correction performance of two $(2048,1365)$ polar codes with different constructions.

Fig. 6.3 shows both the FER (left) and the BER (right) of two different $(2048,1365)$ polar codes. The black-solid curve is the performance of a polar code optimized using the method described in [22] for $E_{b} / N_{0}=4 \mathrm{~dB}$. The dashed-red curve is for the $(2048,1365)$ constructed by assembling (concatenating) a $(1024,512)$ polar code and a $(1024,853)$ polar code. Both polar codes of length 1024 were also optimized using the method of [22] for $E_{b} / N_{0}$ values of 2.5 and 5 $\mathrm{dB}$, respectively.

From the figure, it can be seen that constructing an optimized polar code of length 2048 with 
rate $2 / 3$ results in a coding gain of approximately $0.17 \mathrm{~dB}$ at a FER of $10^{-3}$-an FER appropriate for certain applications-over one assembled from two shorter polar codes of length 1024. The gap is increasing with the signal-to-noise ratio, reaching $0.24 \mathrm{~dB}$ at a FER of $10^{-4}$. Looking at the BER curves, it can be observed that the gap is much narrower. Compared to that of the assembled master code, the optimized polar code shows a coding gain of $0.07 \mathrm{~dB}$ at a BER of $10^{-5}$.

\subsubsection{About Constituent Codes: frozen bit locations, rate and practicality}

The location of the frozen bits in non-optimized constituent codes is dictated by their parent code. In other words, if the master code of length $N$ has been assembled from two optimized (constituent) polar codes of length $N / 2$ as suggested in the previous section, the shorter optimized codes of length $\mathrm{N} / 2$ determine the location of the frozen bits in their respective constituent codes of length $<\mathrm{N} / 2$. Otherwise, the master code dictates the frozen bit locations for all constituent codes.

Assuming that the decoding algorithm takes advantage of the a priori knowledge of these locations, the code rate and frozen bit locations of constituent codes cannot be changed at execution time. However, there are many constituent codes to choose from and code shortening can be used [24] to create more, e.g. in order to obtain a specific number of information bits or code rate.

Because of the polarization phenomenon, given any two sibling constituent codes, the code rate of the LHS one is always lower than that of the RHS one for a properly constructed polar code [25]. That property plays to our advantage as, in many wireless applications, it is desirable to offer a variety of codes of both high and low rates.

It should be noted that not all constituent codes within a master code are of practical use e.g. codes of very high rate offer negligible coding gain over an uncoded communication. For example, among the four constituent codes of length 4 included in the $(16,12)$ polar code illustrated in Fig. 6.1a, two of them are rate-1 constituent codes. Using them would be equivalent to uncoded communication. Moreover, among constituent codes of the same length, many codes may have a similar number of information bits with little to no error-correction performance difference in the region of interest.

Fig. 6.4 shows the frame-error rate of all four constituent codes of length 128 with a rate of approximately $5 / 6$ that are contained within the proposed $(2048,1365)$ master code. It can be seen that, even at such a short length, at a FER of $10^{-3}$ the gap between both extremes is under $0.5 \mathrm{~dB}$. Among those constituent codes, only the $(128,108)$ was selected for the implementation presented in Section 6.5. It is beneficial to limit the number of codes supported in a practical implementation 


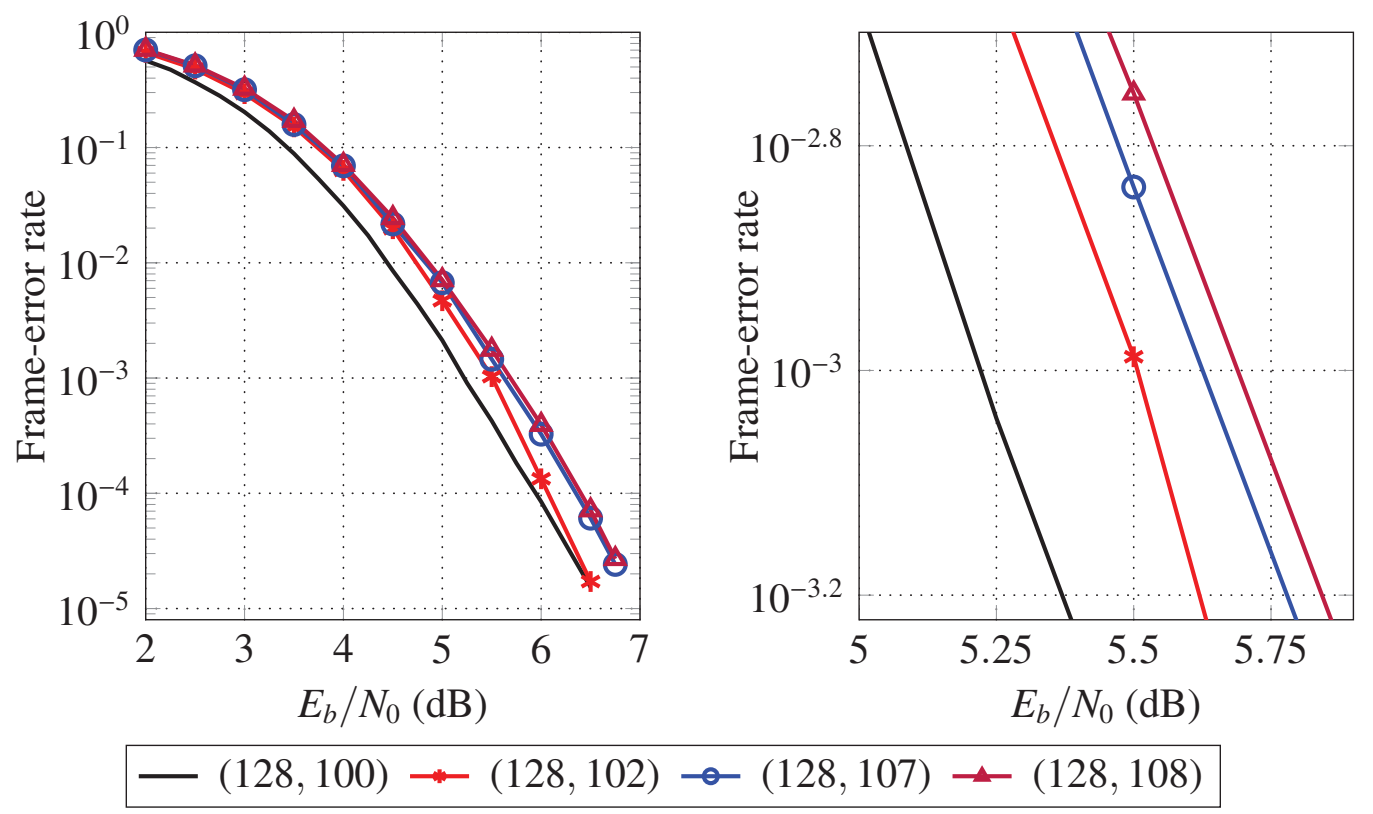

Figure 6.4: Error-correction performance of the four constituent codes of length 128 with a rate of approximately $5 / 6$ contained in the proposed $(2048,1365)$ master code.

of a multi-mode decoder in order to minimize routing circuitry.

\subsubsection{Latency and Throughput Considerations}

If a decoding algorithm taking advantage of the a priori knowledge of the frozen bit locations is used in the unrolled decoder, such as Fast-SSC [8], the latency will vary even among constituent codes of the same length. However, the coded throughput will not. The coded throughput of an unrolled decoder for a polar code of length $N$ will be twice that of a constituent code of $N / 2$, which in turn, is double that of a constituent code of length $N / 4$, and so on.

In an unrolled decoder, the coded and information throughput are defined by the code length $N$, the clock frequency in $\mathrm{Hz} f$, the initiation interval $\mathcal{I}$ and the code rate $R$ [21]. They can be represented as

$$
\mathcal{T}_{C}=\frac{N \cdot f}{\mathcal{I}} \quad \text { and } \quad \mathcal{T}_{I}=\frac{R \cdot N \cdot f}{\mathcal{I}}
$$

respectively.

In wireless communication standards where multiple code lengths and rates are supported, the 
peak information throughput is typically achieved with the longest code that has both the greatest latency and highest code rate. It is not mandatory to reproduce this with our proposed method, but it can be done if considered desirable. It is the example that we provide in the implementation section of this chapter.

Another possible scenario would be to use a low-rate master code, e.g. $R=1 / 3$, that is more powerful in terms of error-correction performance. The resulting multi-mode decoder would reach its peak information throughput with the longest constituent code of length $N / 2$ that has the highest code rate, a code with a significantly lower decoding latency than that of the master code.

\subsection{Implementation Results}

In this section, we present results for two implementations of our proposed multi-mode unrolled decoder with the objective of building decoders with a throughput in the vicinity of $20 \mathrm{Gbps}$. These examples are built around $(1024,853)$ and $(2048,1365)$ master codes. In the following, the former is referred to as the decoder supporting a maximum code length $N_{\max }$ of 1024 and the latter as the decoder with $N_{\max }=2048$. A total of ten polar codes were selected for the decoder supporting codes of lengths up to 2048. The other decoder with $N_{\max }=1024$ has eight modes corresponding to a subset of the ten polar codes supported by the bigger decoder. The master codes used in this section are the same as those used in Section 6.4.2.

For the decoder with $N_{\max }=1024$, the Repetition and SPC nodes were constrained to a maximum size $N_{v}$ of 8 and 4, respectively, as proposed in the previous chapter. For the decoder with $N_{\max }=2048$, we found it more beneficial to lower the execution frequency and increase the maximum sizes of the Repetition and SPC nodes to 16 and 8, respectively. Additionally, the decoder with $N_{\max }=2048$ also uses RepSPC [8] nodes to reduce latency.

ASIC synthesis results are for the $65 \mathrm{~nm}$ CMOS GP technology from TSMC and are obtained with Cadence RTL Compiler. Power consumption estimations are also obtained from Cadence RTL Compiler, switching activity is derived from simulation vectors. Four and five bits of quantization are used for the channel and internal LLRs, respectively. Only registers were used for memory due to the lack of access to an SRAM compiler.

We start by showing the error-correction performance of the various polar codes supported by the implementations. We later present the latency and throughput results for each of these polar codes. This section ends with synthesis results along with power consumption estimations and a 
comparison against the state-of-the-art polar decoder implementations.

\subsubsection{Error-correction Performance}

Fig. 6.5 shows the frame-error rate performance of ten different polar codes. The decoder with $N_{\max }=2048$ supports all ten illustrated polar codes whereas the decoder with $N_{\max }=1024$ supports all polar codes but the two shown as dotted curves. All simulations are generated using random codewords modulated with BPSK and transmitted over an AWGN channel.
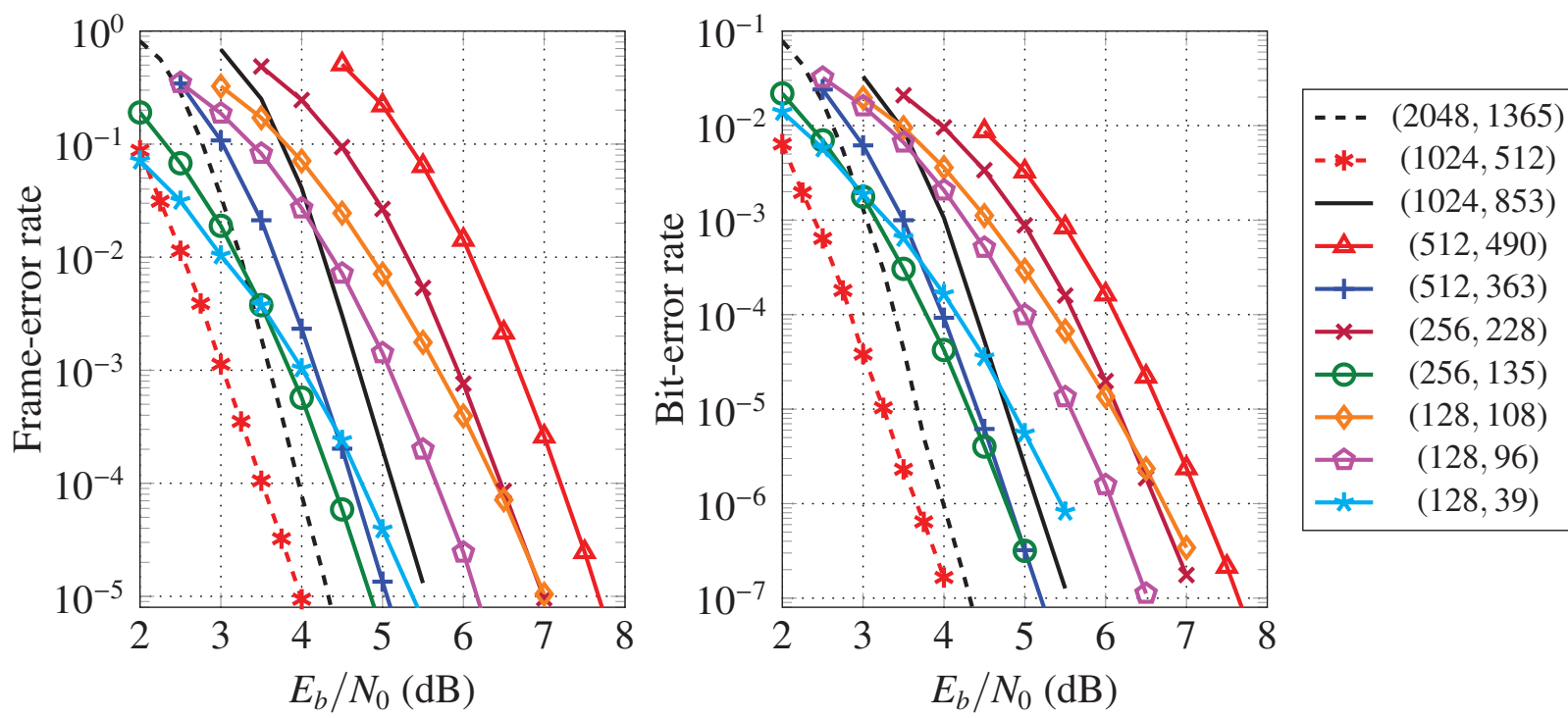

Figure 6.5: Error-correction performance of the polar codes.

It can be seen from the figure that the error-correction performance of the supported polar codes varies greatly. As expected, for codes of the same lengths, the codes with the lowest code rates performs significantly better than their higher rate counterpart. For example, at a FER of $10^{-4}$, the performance of the $(512,363)$ polar code is almost $3 \mathrm{~dB}$ better than that of the $(512,490)$ code.

While the error-correction performance plays a role in the selection of a code, the latency and throughput are also important considerations. As it will be shown in the following section, the ten selected polar codes perform much differently in that regard as well. 


\subsubsection{Latency and Throughput}

Table 6.1 shows the latency and information throughput for both decoders with $N_{\max } \in\{1024,2048\}$. To reduce the area and latency while retaining the same throughput, the initiation interval $\mathcal{I}$ can be increased along with the clock frequency (6.1) [21].

If both decoders have initiation intervals of 20-as used in the section below-Table 6.1 assumes clock frequencies of $500 \mathrm{MHz}$ and $250 \mathrm{MHz}$ for the decoders with $N_{\max }=1024$ and $N_{\max }=2048$, respectively. While their master codes differ, both decoders feature a peak information throughput in the vicinity of $20 \mathrm{Gbps}$. For the decoder with the smallest $N_{\max }$, the seven other polar codes have an information throughput in the multi-gigabit per second range with the exception of the shortest and lowest-rate constituent code. That $(128,39)$ constituent code still has an information throughput close to $1 \mathrm{Gbps}$. The decoder with $N_{\max }=2048$ offers multi-gigabit throughput for most of the supported polar codes. The minimum information throughput is also with the $(128,39)$ polar code at approximately $500 \mathrm{Mbps}$.

Table 6.1: Information throughput and latency for the multi-mode unrolled polar decoders based on the $(2048,1365)$ and $(1024,853)$ master codes, respectively with a $N_{\max }$ of 1024 and 2048.

\begin{tabular}{|c|c|c|c|c|c|c|c|}
\hline \multirow{2}{*}{$\begin{array}{c}\text { Code } \\
(N, k)\end{array}$} & \multirow{2}{*}{$\begin{array}{l}\text { Rate } \\
(k / N)\end{array}$} & \multicolumn{2}{|c|}{ Info. T/P (Gbps) } & \multicolumn{2}{|c|}{ Latency (CCs) } & \multicolumn{2}{|c|}{ Latency (ns) } \\
\hline & & $N_{\max }=1024$ & 2048 & 1024 & 2048 & 1024 & 2048 \\
\hline$(2048,1365)$ & $2 / 3$ & - & 17.1 & - & 503 & - & 2,012 \\
\hline$(1024,853)$ & $5 / 6$ & 21.3 & 10.7 & 323 & 236 & 646 & 944 \\
\hline$(1024,512)$ & $1 / 2$ & - & 6.4 & - & 265 & - & 1,060 \\
\hline$(512,490)$ & $19 / 20$ & 12.3 & 6.2 & 95 & 75 & 190 & 300 \\
\hline$(512,363)$ & $7 / 10$ & 9.1 & 4.5 & 226 & 159 & 452 & 636 \\
\hline$(256,228)$ & $9 / 10$ & 5.7 & 2.6 & 86 & 61 & 172 & 244 \\
\hline$(256,135)$ & $1 / 2$ & 3.4 & 1.7 & 138 & 96 & 276 & 384 \\
\hline$(128,108)$ & $5 / 6$ & 2.7 & 1.4 & 54 & 40 & 108 & 160 \\
\hline$(128,96)$ & $3 / 4$ & 2.4 & 1.2 & 82 & 52 & 164 & 208 \\
\hline$(128,39)$ & $1 / 3$ & 0.98 & 0.49 & 54 & 42 & 108 & 168 \\
\hline
\end{tabular}

In terms of latency, the decoder with $N_{\max }=1024$ requires 646 ns to decode its longest supported code. The latency for all the other codes supported by that decoder is under $500 \mathrm{~ns}$. Even with its additional dedicated node and relaxed maximum size constraint on the Repetition and SPC nodes, the decoder with $N_{\max }=2048$ has greater latency overall because of its lower clock frequency. For example, its latency is of $2.01 \mu \mathrm{s}, 944 \mathrm{~ns}$ and $1.06 \mu \mathrm{s}$ for the $(2048,1365),(1024,853)$ 
and $(1024,512)$ polar codes, respectively.

Using the same nodes and constraints as for $N_{\max }=1024$, the $N_{\max }=2048$ decoder would allow for greater clock frequencies. While $689 \mathrm{CCs}$ would be required to decode the longest polar code instead of 503, a clock of $500 \mathrm{MHz}$ would be achievable, effectively reducing the latency from $2.01 \mu$ s to $1.38 \mu \mathrm{s}$ and doubling the throughput. However, this reduction comes at the cost of much greater area and an estimated power consumption close to $1 \mathrm{~W}$.

\subsubsection{Synthesis Results and Comparison with the State of the Art}

Table 6.2 shows the synthesis results along with power consumption estimations for the two implementations of the proposed multi-mode unrolled decoder. The work in the first two columns is for the decoder with $N_{\max }=1024$, based on the $(1024,853)$ master code. It was synthesized for clock frequencies of $500 \mathrm{MHz}$ and $650 \mathrm{MHz}$, respectively, with initiation intervals $\mathcal{I}$ of 20 and 26. Our work shown in the third and fourth columns is for the decoders with $N_{\max }=2048$, built from the assembled $(2048,1365)$ polar code. These decoders have an initiation interval $\mathcal{I}$ of 20 or 28 , with lower clock frequencies of $250 \mathrm{MHz}$ and $350 \mathrm{MHz}$, respectively. For comparison with other works, the same table also includes results for a dedicated partially-pipelined decoder for a $(1024,512)$ polar code as presented in Chapter 5.

Table 6.2: Comparison with state-of-the-art polar decoders.

\begin{tabular}{|c|c|c|c|c|c|c|c|c|c|}
\hline & \multicolumn{4}{|c|}{ Multi-mode } & Dedicated & [17] & {$[15]^{\diamond}$} & [47] & [37] \\
\hline Algorithm & \multicolumn{4}{|c|}{ Fast-SSC } & Fast-SSC & Fast-SSC & BP & SC & 2-bit SC \\
\hline Technology & \multicolumn{4}{|c|}{$65 \mathrm{~nm}$} & $65 \mathrm{~nm}$ & $65 \mathrm{~nm}$ & $65 \mathrm{~nm}$ & $90 \mathrm{~nm}$ & $45 \mathrm{~nm}$ \\
\hline$N_{\max }$ & \multicolumn{2}{|c|}{1024} & \multicolumn{2}{|c|}{2048} & 1024 & 1024 & 1024 & 1024 & 1024 \\
\hline Code & \multicolumn{2}{|c|}{$(1024,853)$} & \multicolumn{2}{|c|}{$(2048,1365)$} & $(1024,512)$ & $(1024,512)$ & $(1024,512)$ & $(1024, k)$ & $(1024,512)$ \\
\hline Init. Interval $(\mathcal{I})$ & 20 & 26 & 20 & 28 & 20 & - & - & - & - \\
\hline Supply (V) & 0.72 & 1.0 & 0.72 & 1.0 & 1.0 & 1.0 & 1.0 & 1.3 & $\mathrm{~N} / \mathrm{A}$ \\
\hline Oper. temp. $\left({ }^{\circ} \mathrm{C}\right)$ & 125 & 25 & 125 & 25 & 25 & 25 & $\approx 25$ & $\mathrm{~N} / \mathrm{A}$ & $\mathrm{N} / \mathrm{A}$ \\
\hline Area $\left(\mathrm{mm}^{2}\right)$ & 1.71 & 1.44 & 4.29 & 3.58 & 1.68 & 0.69 & 1.48 & 3.21 & $\mathrm{~N} / \mathrm{A}$ \\
\hline Area@65nm $\left(\mathrm{mm}^{2}\right)$ & 1.71 & 1.44 & 4.29 & 3.58 & 1.68 & 0.69 & 1.48 & 1.68 & 0.4 \\
\hline Frequency $(\mathrm{MHz})$ & 500 & 650 & 250 & 350 & 500 & 600 & 300 & 2.5 & 750 \\
\hline Latency $(\mu \mathrm{s})$ & 0.65 & 0.50 & 2.01 & 1.44 & 0.73 & 0.27 & 50 & 0.39 & 1.02 \\
\hline Coded T/P (Gbps) & 25.6 & 25.6 & 25.6 & 25.6 & 25.6 & 3.7 & $4.7 @ 4$ dB & 2.56 & 1.0 \\
\hline Sust. Coded T/P (Gbps) & 25.6 & 25.6 & 25.6 & 25.6 & 25.6 & 3.7 & 2.0 & 2.56 & 1.0 \\
\hline Area Eff. $\left(\mathrm{Gbps} / \mathrm{mm}^{2}\right)$ & 15.42 & 17.75 & 5.97 & 7.16 & 15.27 & 5.40 & $3.18 @ 4 \mathrm{~dB}$ & 0.80 & $\mathrm{~N} / \mathrm{A}$ \\
\hline Power $(\mathrm{mW})$ & 226 & 546 & 379 & 740 & 386 & 215 & 478 & 191 & $\mathrm{~N} / \mathrm{A}$ \\
\hline Energy (pJ/bit) & 8.8 & 21.3 & 14.8 & 28.9 & 15.1 & 57.7 & 102.1 & 74.5 & $\mathrm{~N} / \mathrm{A}$ \\
\hline
\end{tabular}

$\diamond$ Measurement results. 
The four fastest polar decoder implementations from the literature are also included for comparison along with normalized area results. For consistency, only the largest polar code supported by each of our proposed multi-mode unrolled decoders is used and the coded throughput, as opposed to the information one, is compared to match what was done in most of the other works.

From Table 6.2, it can be seen that the area for the proposed decoders with $N_{\max }=1024$ are similar to that of the BP decoder of [15] as well as the normalized area for the unrolled SC decoder from [47]. However, their area is from 2.1 to 2.5 times greater than that of [17]. Comparing the multi-mode decoders, the area for the decoder with $N_{\max }=2048$ is over twice that of the ones with $N_{\max }=1024$, however the master code for the former has twice the length of the latter and supports two more modes.

All proposed decoders have a coded throughput that is an order of magnitude greater than the other works. Latency is one to two orders of magnitude lower than that of the BP decoder. Comparing against the SC decoder of [47], the latency is 1.7 or 3.7 times greater for decoders with an $N_{\max }$ of 1024 and 2048, respectively. It should be noted that the decoder of [47] support codes of any rate, where the proposed multi-mode decoders support a limited number of code rates.

The latency of the proposed decoders is higher than the programmable Fast-SSC decoder of [17]. This is due to greater limitations on the specialized repetition and SPC decoders. The decoder in [17] limits repetition decoders to a maximum length of 32, compared to 8 or 16 in this work, and does not place limits on the SPC decoders.

Finally, among the decoders with $N_{\max }=1024$ implemented in $65 \mathrm{~nm}$ with a power supply of $1 \mathrm{~V}$ and operating at $25^{\circ} \mathrm{C}$, our proposed implementation offers the greatest area and energy efficiency. The proposed multi-mode decoder exhibits 3.3 and 5.6 times better area efficiency than the decoders of [17] and [15], respectively. The energy efficiency is estimated to be 2.7 and 4.8 times higher compared to that of the same two decoders from the literature.

Recently, a List-based multi-mode decoder was proposed in [70], where the definition of the word "multi-mode" differs greatly with our work: in our work, it is used to indicate that the decoder is capable of decoding codes with varying length and rate. Whereas in [70], a "mode" indicates the level of parallelism in the decoder. The decoder of [70] is capable of decoding 4 paths in parallel by implementing 4 processing units. It can be configured to either do SC-based decoding of 4 frames or List-based decoding. For the latter, two list sizes $L$ are supported. If $L=2,2$ frames are decoded in parallel otherwise if $L=4$, only 1 frame is decoded at a time. 


\subsection{Conclusion}

In this chapter we presented a new method to transform an unrolled architecture into a multi-mode decoder supporting various polar code lengths and rates. We showed that a master code can be assembled from two optimized polar codes of smaller length, with desired code rates, without sacrificing too much coding gain. We provided results for two decoders, one built for a $(1024,853)$ master code and the other for a longer $(2048,1365)$ polar code. Both decoders support from seven to nine other practical codes. On $65 \mathrm{~nm}$ ASIC, they were shown to have a peak throughput greater than $25 \mathrm{Gbps}$. One has a worst-case latency of $2 \mu \mathrm{s}$ at $250 \mathrm{MHz}$ and an energy efficiency of $14.8 \mathrm{pJ} / \mathrm{bit}$. The other has a worst-case latency of $646 \mathrm{~ns}$ at $500 \mathrm{MHz}$ and an energy efficiency of $8.8 \mathrm{pJ} / \mathrm{bit}$. Both implementation examples show that, with their great throughput and support for codes of various lengths and rates, multi-mode unrolled polar decoders are promising candidates for future wireless communication standards. 



\section{Chapter 7}

\section{Conclusion and Future Work}

Error-correcting codes play a crucial role in reliable and robust communication and storage systems. The dream of researchers would be to achieve the channel capacity at low implementation complexity without compromising the latency and throughput requirements of living a modern connected life. Polar codes are the latest class of modern error-correcting codes and they show high potential. The early decoder implementations greatly suffered from high latency and low throughput. The state-of-the-art low-complexity algorithm improved the situation but contained improvements targeted at high-rate codes and the throughput was still an order of magnitude lower than the expected requirements for future wireless communication standards. Initially there was a lack of software implementations suitable for high-performance SDR applications and then the work that appeared suffered from a high latency and memory footprint. This thesis presented solutions to address those issues.

The optimization presented in the original Fast-SSC algorithm [8], the fastest low-complexity decoding algorithm, targeted high-rate codes. In Chapter 3, we showed how to improve the FastSSC algorithm by adding dedicated decoders for three new types of constituent codes frequently appearing in low-rate codes. We also introduced a human-guided polar code construction alteration method to significantly reduce the latency and increase the throughput of a Fast-SSC decoder at the cost of a small error-correction performance loss. The resulting decoders for codes of rate $1 / 2$ and $1 / 3$ presented in this work achieved an information throughput greater $1.2 \mathrm{Gbps}$ at an operating frequency of $400 \mathrm{MHz}$, while retaining the low complexity of the original Fast-SSC implementation.

With their low-complexity encoding and decoding algorithms, polar codes are attractive for 
applications where computational resources are limited and a custom hardware solution too costly. Chapter 4 presented low-latency software polar decoders exploiting the capabilities offered in modern processors. By adapting the algorithms at various levels, the software decoders presented in this work had an order of magnitude lower latency and memory footprint compared to the state-ofthe-art decoders, while maintaining a comparable throughput. In addition, we presented strategies for implementing polar decoders on graphical processing units and showed that hundreds of frames could be simultaneously decoded while sustaining a throughput greater than $1 \mathrm{Gbps}$.

Chapter 5 introduced a family of hardware architectures using a reduced-complexity successivecancellation decoding algorithm that employs unrolling. It demonstrated that polar decoders can achieve extremely high throughput values and retain moderate complexity. The resulting fullyunrolled architectures were shown to be capable of achieving a throughput that is two to three orders of magnitude greater than current state-of-the-art polar decoders, while retaining a good energy efficiency.

Many communication standards mandate the error-correction system to support various code lengths and rate in order to adapt to varying channel conditions or latency requirements. Multimode unrolled hardware architectures and implementations were proposed in Chapter 6. This novel method lead to a length- and rate-flexible decoder while retaining the very high speed typical to unrolled decoders. Results were presented for two versions of a multi-mode decoder supporting eight and ten different polar codes, respectively. Both showed that, with their throughput greater than $25 \mathrm{Gbps}$, latency below $2 \mu \mathrm{s}$ and support for codes of various lengths and rates, multi-mode unrolled polar decoders are promising candidates for future wireless communication standards.

\subsection{Future Work}

The research presented in this thesis showed that polar codes are a new class of modern errorcorrecting codes that already show great potential for use in some practical applications. For example, encoding and decoding of polar codes on modern processors for use in SDR applications already makes sense. However, the software implementations we presented were not suitable for micro-controller processors omnipresent in Internet of Things (IoT) devices. Also, as the errorcorrection performance of moderate-length polar codes - when an SC-based decoding algorithm is used - is less than that of LDPC codes, polar codes will not take over the world of error correction just yet. List-based decoding can close that gap [33] but its hardware implementations suffer from 
low throughput [71], [72] even if there is hope for improvements [73], [74]. Here is a list of suggested future research topics that would help broaden the scope of interesting applications for polar codes.

\subsubsection{Software Encoding and Decoding on APU Processors}

The GPU implementation results presented in Chapter 4 showed that hundreds of frames could be simultaneously decoded at a sustained throughput greater than 1 Gbps. That throughput was shown to be I/O bound to the capabilities of the PCIe bus. Even with a GPU and motherboard supporting a faster, more recent, iteration of the PCIe, the memory copy latency for moving data from the host memory to the card memory will remain a significant barrier to a better throughput. There exists processors coupled with a GPGPU on the same die sharing the same memory. AMD's APU are among those. It would be interesting to investigate the use of APUs to conduct software decoding of polar codes.

\subsubsection{Software Encoding and Decoding on Micro-controllers}

A great share of the optimization strategies presented in Chapter 4 cannot be applied to microcontrollers, processors that do not have SIMD instructions. Most IoT devices of today either use micro-controllers or a SoC that features one because of their relative low cost. While these devices are not powerful enough to implement a practical LDPC or turbo decoder, an error-correction solution based on polar codes instead of a classic codes such as BCH or RS codes is certainly an interesting avenue to explore.

\subsubsection{High-speed Systematic Encoder}

Throughout this thesis we have introduced decoders capable for very-high throughput e.g. unrolled decoders with a throughput beyond 1 Tbps in Chapter 5. The fastest encoder for systematic polar codes from the literature, [25], achieves a throughput well under $100 \mathrm{Gbps}$. One possible research avenue would be to investigate if, as hinted by the techniques used in fast decoding of polar codes, taking advantage of the a priori knowledge of the frozen bit locations could lead to orders of magnitude throughput improvement and latency reduction by simplifying the encoding algorithm. 


\subsubsection{Multi-mode Unrolled List Decoders}

The focus is shifting away from SC-based decoding in favor of List-based decoding as CRC-aided List decoding of polar codes can outperform the error-correction performance of LDPC codes. However, the current state of the art in hardware List decoders suffers from a low throughput and high latency. The multi-mode unrolled hardware architectures in Chapter 6 can be applied to List-based decoding. In order to keep complexity practical, it would be interesting to adapt and implement such architectures for small list sizes. 


\section{Bibliography}

[1] C. Shannon, "A mathematical theory of communication", Bell Syst. Tech. J., vol. 27, no. 3, pp. 379-423, Jul. 1948, IssN: 0005-8580. Dor: 10.1002/j . 1538-7305 . 1948 . tb01338 . x.

[2] C. Berrou, A. Glavieux, and P. Thitimajshima, "Near Shannon limit error-correcting coding and decoding: turbo-codes", in IEEE Int. Conf. Commun. (ICC), vol. 2, May 1993, pp. 10641070. DoI: 10.1109/ICC. 1993.397441.

[3] R. Gallager, "Low-density parity-check codes", IRE Trans. Inf. Theory, vol. 8, no. 1, pp. 2128, Jan. 1962, ISSN: 0096-1000. Dor: 10.1109/TIT . 1962 . 1057683.

[4] D. J. C. MacKay and R. M. Neal, "Near shannon limit performance of low density parity check codes", IET Electron. Lett., vol. 33, no. 6, pp. 457-458, Mar. 1997, ISSN: 0013-5194. Dor: $10.1049 / \mathrm{el}: 19970362$.

[5] E. Arıkan, "Channel polarization: a method for constructing capacity-achieving codes", in IEEE Int. Symp. on Inf. Theory (ISIT), Jul. 2008, pp. 1173-1177. Dor: 10 . 1109 / ISIT . 2008.4595172.

[6] E. Arıkan, "Channel polarization: a method for constructing capacity-achieving codes for symmetric binary-input memoryless channels", IEEE Trans. Inf. Theory, vol. 55, no. 7, pp. 3051-3073, 2009. Dor: 10.1109/TIT.2009. 2021379.

[7] A. Alamdar-Yazdi and F. R. Kschischang, "A simplified successive-cancellation decoder for polar codes”, IEEE Commun. Lett., vol. 15, no. 12, pp. 1378-1380, 2011. DoI: 10 . 1109/ LCOMM . 2011.101811 .111480$.

[8] G. Sarkis, P. Giard, A. Vardy, C. Thibeault, and W. J. Gross, "Fast polar decoders: algorithm and implementation", IEEE J. Sel. Areas Commun., vol. 32, no. 5, pp. 946-957, May 2014, ISSN: 0733-8716. DoI: 10.1109/JSAC.2014.140514. arXiv: 1307.7154.

[9] K. Tan, H. Liu, J. Zhang, Y. Zhang, J. Fang, and G. M. Voelker, "Sora: high-performance software radio using general-purpose multi-core processors", Commun. ACM, vol. 54, no. 1, pp. 99-107, Jan. 2011, ISSN: 0001-0782. DoI: 10. 1145/1866739. 1866760.

[10] J. Demel, S. Koslowski, and F. Jondral, "A LTE receiver framework using GNU Radio", J. Signal Process. Syst., vol. 78, no. 3, pp. 313-320, 2015, ISSN: 1939-8018. DoI: 10.1007 / s11265-014-0959-z. 
[11] J. Xianjun, C. Canfeng, P. Jaaskelainen, V. Guzma, and H. Berg, "A 122Mb/s turbo decoder using a mid-range GPU”, in Int. Wireless Commun. and Mobile Comput. Conf. (IWCMC), Jul. 2013, pp. 1090-1094. Dor: 10.1109/IWCMC. 2013.6583709.

[12] G. Wang, M. Wu, B. Yin, and J. R. Cavallaro, "High throughput low latency LDPC decoding on GPU for SDR systems", in IEEE Glob. Conf. on Sign. and Inf. Process. (GlobalSIP), Dec. 2013, pp. 1258-1261. DoI: 10.1109/GlobalSIP.2013.6737137.

[13] B. Le Gal, C. Jego, and J. Crenne, "A high throughput efficient approach for decoding LDPC codes onto GPU devices", IEEE Embedded Syst. Lett., vol. 6, no. 2, pp. 29-32, Jun. 2014, ISSN: 1943-0663. DOI: 10.1109/LES . 2014 .2311317.

[14] X. Han, K. Niu, and Z. He, "Implementation of IEEE 802.11n LDPC codes based on general purpose processors", in IEEE Int. Conf. on Commun. Technol. (ICCT), Nov. 2013, pp. 218 222. DoI: $10.1109 /$ ICCT . 2013.6820375.

[15] Y. S. Park, Y. Tao, S. Sun, and Z. Zhang, “A 4.68Gb/s belief propagation polar decoder with bit-splitting register file”, in Symp. on VLSI Circ. Dig. of Tech. Papers, Jun. 2014, pp. 1-2. DOI: $10.1109 /$ VLSIC . 2014.6858413.

[16] P. Giard, G. Sarkis, C. Thibeault, and W. J. Gross, “A 638 Mbps low-complexity rate 1/2 polar decoder on FPGAs", in IEEE Int. Workshop on Signal Process. Syst. (SiPS), Hangzhou, CHN, Oct. 2015, pp. 1-6. Dor: 10.1109/SiPS . 2015. 7345007.

[17] P. Giard, A. Balatsoukas-Stimming, G. Sarkis, C. Thibeault, and W. J. Gross, "Fast lowcomplexity decoders for low-rate polar codes", Springer J. Signal Process. Syst., 2016. DoI: 10.1007 / s11265-016-1173-y. arXiv: 1603.05273 , invited, to appear and prepublished.

[18] P. Giard, G. Sarkis, C. Thibeault, and W. J. Gross, "Fast software polar decoders", in IEEE Int. Conf. on Acoustics, Speech, and Signal Process. (ICASSP), Florence, ITA, May 2014, pp. 7555-7559. Dor: 10.1109/ICASSP. 2014.6855069. arXiv: 1306.6311.

[19] P. Giard, G. Sarkis, C. Leroux, C. Thibeault, and W. J. Gross, "Low-latency software polar decoders", Springer J. Signal Process. Syst., 2016, Issn: 1939-8115. Dor: 10 . 1007/s11265016-1157-y, to appear and pre-published.

[20] P. Giard, G. Sarkis, C. Thibeault, and W. J. Gross, "237 Gbit/s unrolled hardware polar decoder”, IET Electron. Lett., vol. 51, no. 10, pp. 762-763, May 2015, IssN: 0013-5194. Dor: 10.1049/el.2014.4432. arXiv: 1412.6043.

[21] P. Giard, G. Sarkis, C. Thibeault, and W. J. Gross, "Multi-mode unrolled hardware architectures for polar decoders", IEEE Trans. Circuits Syst. I, vol. 63, no. 9, pp. 1443-1453, Sep. 2016, ISSN: 1549-8328. DOI: 10.1109/TCSI . 2016.2586218. arXiv: 1505.01459.

[22] I. Tal and A. Vardy, "How to construct polar codes", IEEE Trans. Inf. Theory, vol. 59, no. 10, pp. 6562-6582, Oct. 2013, ISSN: 0018-9448. DoI: 10. 1109/TIT . 2013.2272694. 
[23] E. Arıkan, "Systematic polar coding", IEEE Commun. Lett., vol. 15, no. 8, pp. 860-862, 2011. DOI: 10.1109 /LCOMM. 2011.061611 .110862$.

[24] Y. Li, H. Alhussien, E. Haratsch, and A. Jiang, "A study of polar codes for MLC NAND flash memories", in Int. Conf. on Comput., Netw. and Commun. (ICNC), Feb. 2015, pp. 608612. DOI: $10.1109 /$ ICCNC. 2015.7069414.

[25] G. Sarkis, I. Tal, P. Giard, A. Vardy, C. Thibeault, and W. J. Gross, "Flexible and lowcomplexity encoding and decoding of systematic polar codes", IEEE Trans. Commun., vol. 64, no. 7, pp. 2732-2745, Jul. 2016, ISSN: 0090-6778. DoI: 10 . 1109 / TCOMM . 2016. 2574996. arXiv: 1507.03614.

[26] G. Sarkis, "Efficient encoders and decoders for polar codes: algorithms and implementations", PhD thesis, McGill University, 2016.

[27] G. Sarkis and W. J. Gross, "Increasing the throughput of polar decoders", IEEE Commun. Lett., vol. 17, no. 4, pp. 725-728, 2013, IssN: 1089-7798. Dor: 10 . 1109 / LCOMM . 2013 . Q21213. 121633.

[28] B. Li, H. Shen, D. Tse, and W. Tong, "Low-latency polar codes via hybrid decoding", in Int. Symp. on Turbo Codes and Iterative Inf. Process. (ISTC), Aug. 2014, pp. 223-227. Dor: 10.1109/ISTC. 2014.6955118.

[29] S. Kahraman and M. E. Çelebi, "Code based efficient maximum-likelihood decoding of short polar codes", in IEEE Int. Symp. on Inf. Theory (ISIT), Jul. 2012, pp. 1967-1971. DoI: 10.1109/ISIT . 2012.6283643.

[30] N. Goela, S. B. Korada, and M. Gastpar, "On lp decoding of polar codes", in IEEE Inf. Theory Workshop (ITW), Aug. 2010, pp. 1-5. Dor: 10.1109/CIG .2010. 5592698.

[31] N. Hussami, R. Urbanke, and S. B. Korada, "Performance of polar codes for channel and source coding", in IEEE Int. Symp. on Inf. Theory (ISIT), 2009, pp. 1488-1492. DoI: 10 . 1109/ISIT . 2009. 5205860 .

[32] B. Yuan and K. K. Parhi, "Early stopping criteria for energy-efficient low-latency beliefpropagation polar code decoders", IEEE Trans. Signal Process., vol. 62, no. 24, pp. 64966506, Dec. 2014. DoI: 10.1109/TSP . 2014.2366712.

[33] I. Tal and A. Vardy, "List decoding of polar codes", IEEE Trans. Inf. Theory, vol. 61, no. 5, pp. 2213-2226, May 2015, IssN: 0018-9448. Dor: 10.1109/TIT . 2015 . 2410251.

[34] C. Leroux, I. Tal, A. Vardy, and W. J. Gross, "Hardware architectures for successive cancellation decoding of polar codes", in IEEE Int. Conf. on Acoust., Speech and Signal Process. (ICASSP), 2011, pp. 1665-1668. Dor: 10.1109/ICASSP . 2011.5946819. 
[35] A. Mishra, A. Raymond, L. Amaru, G. Sarkis, C. Leroux, P. Meinerzhagen, A. Burg, and W. Gross, "A successive cancellation decoder ASIC for a 1024-bit polar code in 180nm CMOS”, in IEEE Asian Solid State Circuits Conf. (A-SSCC), 2012, pp. 205-208. Dor: 10 . 1109/IPEC. 2012.6522661.

[36] A. J. Raymond and W. J. Gross, "Scalable successive-cancellation hardware decoder for polar codes", in IEEE Glob. Conf. on Signal and Inf. Process. (GlobalSIP), Dec. 2013, pp. 1282-1285. DoI: 10.1109/GlobalSIP. 2013.6737143.

[37] B. Yuan and K. Parhi, "Low-latency successive-cancellation polar decoder architectures using 2-bit decoding”, IEEE Trans. Circuits Syst. I, vol. 61, no. 4, pp. 1241-1254, Apr. 2014, ISSN: 1549-8328. DOI: 10.1109/TCSI . 2013. 2283779.

[38] C. Leroux, A. Raymond, G. Sarkis, and W. Gross, "A semi-parallel successive-cancellation decoder for polar codes”, IEEE Trans. Signal Process., vol. 61, no. 2, pp. 289-299, 2013, ISSN: 1053-587X. DOI: 10.1109/TSP. 2012.2223693.

[39] C. Leroux, A. J. Raymond, G. Sarkis, I. Tal, A. Vardy, and W. J. Gross, "Hardware implementation of successive-cancellation decoders for polar codes", J. Signal Process. Syst., vol. 69, no. 3, pp. 305-315, 2012. Dor: $10.1007 /$ s 11265-012-0685-3.

[40] A. Pamuk and E. Arıkan, "A two phase successive cancellation decoder architecture for polar codes”, in IEEE Int. Symp. on Inf. Theory (ISIT), Jul. 2013, pp. 1-5. Dor: 10.1109/ ISIT . 2013.6620368.

[41] A. J. Raymond, "Design and hardware implementation of decoder architectures for polar codes", Master's thesis, McGill University, 2014.

[42] R. Mori and T. Tanaka, "Performance and construction of polar codes on symmetric binaryinput memoryless channels", in IEEE Int. Symp. on Inf. Theory (ISIT), 2009, pp. 1496-1500. DOI: $10.1109 /$ ISIT . 2009.5205857.

[43] P. Trifonov, "Efficient design and decoding of polar codes", IEEE Trans. Commun., vol. 60, no. 11, pp. 3221-3227, 2012. Dor: 10.1109/TCOMM. 2012.081512 . 110872.

[44] Z. Huang, C. Diao, and M. Chen, "Latency reduced method for modified successive cancellation decoding of polar codes", IET Electron. Lett., vol. 48, no. 23, pp. 1505-1506, Nov. 2012, ISSN: 0013-5194. DoI: 10.1049/el .2012 . 2795.

[45] A. Balatsoukas-Stimming, G. Karakonstantis, and A. Burg, "Enabling complexity-performance trade-offs for successive cancellation decoding of polar codes", in IEEE Int. Symp. on Inf. Theory (ISIT), 2014, pp. 2977-2981. DoI: 10.1109/ISIT . 2014.6875380.

[46] L. Zhang, Z. Zhang, X. Wang, C. Zhong, and L. Ping, "Simplified successive-cancellation decoding using information set reselection for polar codes with arbitrary blocklength", IET Communications, vol. 9, no. 11, pp. 1380-1387, Jul. 2015. Dor: 10.1049/i et-com. 2014 . 0988. 
[47] O. Dizdar and E. Arıkan, "A high-throughput energy-efficient implementation of successive cancellation decoder for polar codes using combinational logic", IEEE Trans. Circuits Syst. I, vol. 63, no. 3, pp. 436-447, Mar. 2016, ISSN: 1549-8328. DoI: 10 . 1109 / TCSI . 2016. 2525020 .

[48] B. Le Gal, C. Leroux, and C. Jego, "Software polar decoder on an embedded processor", in IEEE Int. Workshop on Signal Process. Syst. (SiPS), Belfast, UK, Oct. 2014. Dor: 10. 1109/ SiPS. 2014.6986083.

[49] B. Le Gal, C. Leroux, and C. Jego, "Multi-Gb/s software decoding of polar codes", IEEE Trans. Signal Process., vol. 63, no. 2, pp. 349-359, Jan. 2015. Dor: 10. 1109/TSP . 2014. 2371781.

[50] G. Sarkis, P. Giard, C. Thibeault, and W. J. Gross, "Autogenerating software polar decoders", in IEEE Global Conf. on Signal and Inf. Process. (GlobalSIP), Atlanta, USA, Dec. 2014, pp. 6-10. DoI: 10.1109/GlobalSIP. 2014.7032067.

[51] S. Bang, C. Ahn, Y. Jin, S. Choi, J. Glossner, and S. Ahn, "Implementation of LTE system on an SDR platform using CUDA and UHD", Analog Integr. Circuits and Signal Process., vol. 78, no. 3, pp. 599-610, 2014. Dor: 10.1007/s10470-013-0229-1.

[52] "IEEE standard for floating-point arithmetic", IEEE Std 754-2008, pp. 1-70, Aug. 2008. DOI: 10.1109/IEEESTD . 2008 . 4610935.

[53] NVIDIA, "Performance guidelines", CUDA C Programming Guide, Aug. 2014.

[54] W.-C. Feng and S. Xiao, “To GPU synchronize or not GPU synchronize?", in IEEE Int. Symp. on Circuits and Syst. (ISCAS), May 2010, pp. 3801-3804. Dor: 10 . 1109/ISCAS . 2010.5537722 .

[55] NVIDIA, "Kepler GK110 - the fastest, most efficient HPC architecture ever built", NVIDIA's Next Generation CUDA Computer Architecture: Kepler GK110, Dec. 2012.

[56] “PCI express base specification revision 2.0”, PCI-SIG, Dec. 2006.

[57] J. Treibig, G. Hager, and G. Wellein, "LIKWID: a lightweight performance-oriented tool suite for x 86 multicore environments", in Int. Conf. on Parallel Process. Workshops (ICPPW), Sep. 2010, pp. 207-216. Dor: 10.1109/ICPPW . 2010. 38.

[58] NVIDIA, "NVIDIA management library (NVML)", NVML API Reference Guide, Mar. 2014.

[59] P. Jouguet and S. Kunz-Jacques, "High performance error correction for quantum key distribution using polar codes", Quantum Inf. E Computation, vol. 14, no. 3-4, pp. 329-338, 2014. 
[60] "IEEE standard for information technology-telecommunications and information exchange between systems local and metropolitan area networks-specific requirements part 11: wireless LAN medium access control (MAC) and physical layer (PHY) specifications", IEEE Std 802.11-2012 (Revision of IEEE Std 802.11-2007), pp. 1-2793, Mar. 2012. Dor: 10 . 1109/ IEEESTD . 2012.6178212.

[61] W. Roh, " $5 \mathrm{G}$ mobile communications for 2020 and beyond - vision and key enabling technologies", IEEE Wireless Commun. and Netw. Conf. (WCNC), Apr. 2014.

[62] J. Karjalainen, M. Nekovee, H. Benn, W. Kim, J. Park, and H. Sungsoo, "Challenges and opportunities of mm-wave communication in 5G networks", in Int. Conf. on Cognitive Radio Oriented Wireless Netw. and Commun. (CROWNCOM), Jun. 2014, pp. 372-376. Dor: 10.4108/icst. crowncom.2014.255604.

[63] J. F. Monserrat, G. Mange, V. Braun, H. Tullberg, G. Zimmermann, and Ö. Bulakci, "METIS research advances towards the 5G mobile and wireless system definition", EURASIP J. Wireless Commun. Netw., vol. 2015, no. 1, pp. 1-16, 2015. Dor: 10.1186/s13638-015-03029.

[64] P. Schläfer, N. Wehn, M. Alles, and T. Lehnigk-Emden, "A new dimension of parallelism in ultra high throughput LDPC decoding", in IEEE Workshop on Signal Process. Syst. (SiPS), 2013, pp. 153-158. Dor: 10.1109/SiPS.2013.6674497.

[65] N. Wehn, S. Scholl, P. Schläfer, T. Lehnigk-Emden, and M. Alles, "Challenges and limitations for very high throughput decoder architectures for soft-decoding", in Advanced Hardware Design for Error Correcting Codes, C. Chavet and P. Coussy, Eds., Springer International Publishing, 2015, pp. 7-31, IsBn: 978-3-319-10568-0. Dor: 10 . 1007/978-3-31910569-7_2.

[66] R. Wang and R. Liu, "A novel puncturing scheme for polar codes", IEEE Commun. Lett., vol. 18, no. 12, pp. 2081-2084, Dec. 2014, ISSN: 1089-7798. Dor: 10 . 1109/LCOMM. 2014. 2364845 .

[67] V. Miloslavskaya, "Shortened polar codes", IEEE Trans. Inf. Theory, vol. 61, no. 9, pp. 48524865, Sep. 2015, ISSN: 0018-9448. Dor: 10.1109/TIT . 2015.2453312.

[68] Xilinx, "UltraScale architecture and product overview", Product Specification, Dec. 2014.

[69] Altera, "Meeting the performance and power imperative of the zettabyte era with generation 10", White Paper, Jun. 2013.

[70] C. Xiong, J. Lin, and Z. Yan, "A multimode area-efficient SCL polar decoder", IEEE Trans. VLSI Syst., vol. PP, no. 99, pp. 1-14, 2016, ISSN: 1063-8210. Dor: 10 . 1109/TVLSI. 2016. 2557806.

[71] B. Li, H. Shen, and D. Tse, "An adaptive successive cancellation list decoder for polar codes with cyclic redundancy check", IEEE Commun. Lett., vol. 16, no. 12, pp. 2044-2047, Dec. 2012, ISSN: 1089-7798. DOI: 10.1109/LCOMM. 2012 .111612 . 121898. 
[72] A. Balatsoukas-Stimming, M. Bastani Parizi, and A. Burg, "LLR-based successive cancellation list decoding of polar codes", IEEE Trans. Signal Process., vol. 63, no. 19, pp. 51655179, Oct. 2015, ISSN: 1053-587X. DoI: 10.1109/TSP . 2015 . 2439211.

[73] G. Sarkis, P. Giard, A. Vardy, C. Thibeault, and W. J. Gross, "Fast list decoders for polar codes", IEEE J. Sel. Areas Commun. - Special Issue on Recent Advances In Capacity Approaching Codes, vol. 34, no. 2, pp. 318-328, Feb. 2016, Issn: 0733-8716. Dor: 10. 1109 / JSAC. 2015.2504299 . arXiv: 1505.01466.

[74] S. A. Hashemi, A. Balatsoukas-Stimming, P. Giard, C. Thibeault, and W. J. Gross, "Partitioned successive-cancellation list decoding of polar codes", in IEEE Int. Conf. on Acoustics, Speech, and Signal Process. (ICASSP), Shanghai, CHN, Mar. 2016, pp. 957-960. Dor: 10.1109/ICASSP . 2016.7471817. 



\section{List of Acronyms}

ASIC Application-Specific Integrated Circuit. 4, 5, 7, 8, 23, 24, 37, 40, 67-69, 76-79, 81, 82, $85-88,94$

AVX Advanced Vector eXtensions. 6, 44, 46, 49, 54, 64

AWGN Additive White Gaussian-Noise. 35, 43, 76, 95

BCH Bose-Chaudhuri-Hocquenghem. 1, 103

BER Bit-Error Rate. 11, 76, 91, 92

BP Belief Propagation. 3, 17, 18, 69, 78

BPSK Binary Phase-Shift Keying. 35, 43, 76, 95

CC Clock Cycle. 24, 30, 32, 83, 90, 97

CPU Central Processing Unit. 41, 42, 44, 46, 52-56

CRC Cyclic Redundancy Check. 18

DRAM Dynamic Random-Access Memory. 61

Fast-SSC Fast Simplified Successive Cancellation. 2, 3, 5, 6, 14, 16, 19, 20, 22-24, 26, 30, 32, $35-37,39,43,44,53,68-70,74,89,93,101$

FER Frame-Error Rate. 11, 44, 63, 91, 92, 95

FPGA Field-Programmable Gate-Array. 4, 6, 7, 20, 23, 24, 35, 36, 40, 44, 62, 67-71, 76-78, $80-85$ 
GPGPU General Purpose GPU. 7, 55, 103

GPU Graphical Processing Unit. 6, 42, 47, 55-62, 64, 103

I/O Input/Output. 34, 67, 103

IoT Internet of Things. 102, 103

LDPC Low-Density Parity-Check. 1, 2, 17, 18, 41, 42, 63, 69, 103, 104

LHS Left-Hand-Side. 88, 91, 92

LLR Log-Likelihood Ratio. 12-17, 20, 33, 34, 44-46, 60, 62, 71, 73-76, 83-85, 89, 90, 94

LUT Look-Up Table. 36, 70, 76-78, 80, 81, 90

ML maximum-likelihood. 16, 17

PE Processing Element. 19, 20

RAM Random-Access Memory. 20, 34, 36, 77, 80, 81

RHS Right-Hand-Side. 88, 91, 92

RS Reed-Solomon. 1, 103

RTL Register-Transfer Level. 35

SC Successive-Cancellation. 2, 3, 12-14, 16-20, 25, 53, 68, 69, 80, 102, 104

SDR Software-Defined Radio. 2, 4, 41-44, 53, 54, 62, 65, 101, 102

SIMD Single-Instruction Multiple-Data. 2, 6, 7, 41-45, 48, 49, 51, 54, 55, 64, 103

SoC System on Chip. 54, 61, 103

SPC Single Parity Check. 15, 24, 25, 28, 45, 49, 57, 74, 83, 90, 94, 96

SP-SC Semi-Parallel Successive-Cancellation. 20, 22 
SRAM Static Random-Access Memory. 37, 71, 75-77, 94

SSC Simplified Successive Cancellation. 2, 14, 16, 17, 53

SSE Streaming SIMD Extensions. 6, 44, 46, 49, 54, 64

TP-SC Two-Phase Successive-Cancellation. 20-22 